\title{
Common carotid artery morphology and dynamics estimated with automatic ultrasound segmentation
}

Citation for published version (APA):

Rossi, A. C. (2009). Common carotid artery morphology and dynamics estimated with automatic ultrasound segmentation. [Doctoral Thesis, Maastricht University]. Datawyse / Universitaire Pers Maastricht. https://doi.org/10.26481/dis.20091105ar

Document status and date:

Published: 01/01/2009

DOI:

10.26481/dis.20091105ar

Document Version:

Publisher's PDF, also known as Version of record

\section{Please check the document version of this publication:}

- A submitted manuscript is the version of the article upon submission and before peer-review. There can be important differences between the submitted version and the official published version of record.

People interested in the research are advised to contact the author for the final version of the publication, or visit the DOI to the publisher's website.

- The final author version and the galley proof are versions of the publication after peer review.

- The final published version features the final layout of the paper including the volume, issue and page numbers.

Link to publication

\footnotetext{
General rights rights.

- You may freely distribute the URL identifying the publication in the public portal. please follow below link for the End User Agreement:

www.umlib.nl/taverne-license

Take down policy

If you believe that this document breaches copyright please contact us at:

repository@maastrichtuniversity.nl

providing details and we will investigate your claim.
}

Copyright and moral rights for the publications made accessible in the public portal are retained by the authors and/or other copyright owners and it is a condition of accessing publications that users recognise and abide by the legal requirements associated with these

- Users may download and print one copy of any publication from the public portal for the purpose of private study or research.

- You may not further distribute the material or use it for any profit-making activity or commercial gain

If the publication is distributed under the terms of Article $25 \mathrm{fa}$ of the Dutch Copyright Act, indicated by the "Taverne" license above, 
Common carotid artery morphology and dynamics estimated with automatic ultrasound segmentation

Alessandro C. Rossi 
ISBN 978-90-9024620-8

(C) copyright Alessandro C. Rossi, Maastricht 2009

Cover design by Alessandro C. Rossi

Front cover: carotid artery drawing

Back cover: processed image of the Maas river (bifurcation, Maastricht north)

Printing: Datawyse / Universitaire Pers Maastricht 


\title{
Common carotid artery morphology and dynamics estimated with automatic ultrasound segmentation
}

\author{
DISSERTATION \\ to obtain the degree of Doctor at the Maastricht University, \\ on the authority of the Rector Magnificus Prof. dr. G.P.M.F. Mols, \\ in accordance with the decision of the Board of Deans, \\ to be defended in public on Thursday $5^{\text {th }}$ November 2009, at 16:00 hours \\ by \\ Alessandro C. Rossi \\ born on $9^{\text {th }}$ June 1981 in Piacenza, Italy
}




\section{Supervisor}

Prof. Dr. Ir. Arnold P.G. Hoeks

\section{Co-supervisor}

Dr. Ing. Peter J. Brands

\section{Assessment Committee}

Prof. Dr. Harry A.J. Struijker Boudier (chairman)

Prof. Dr. Pierre Boutouyrie

Prof. Dr. Werner H. Mess

Prof. Dr. Robert S. Reneman

Prof. Dr. Ir. Patrick Segers

This research project has been supported by a Marie Curie Early Stage Research Training Fellowship of the European Community's Sixth Framework Programme under contract number MEST-CT-2004-514421.

Financial support by the Netherlands Heart Foundation and Stichting Hartsvrienden RESCAR for the publication of this thesis is gratefully acknowledged.

Financial support for the dissertation costs as provided by:

- Esaote Europe

- Esaote Pie Medical Benelux

- Medtronic Bakken Research Center

- AstraZeneca

- Pfizer

is gratefully acknowledged. 


\section{CONTENTS}

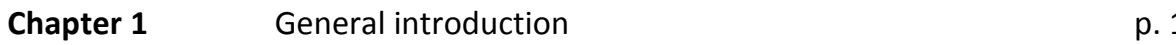

$\begin{array}{lll}\text { Chapter } 2 & \text { Medical ultrasound: physics and technology } & \text { p. } 17\end{array}$

$\begin{array}{lll}\text { Chapter } 3 & \text { Effects of non-linear processing on common carotid } & \text { p. } 37\end{array}$ artery diameter estimation

$\begin{array}{lll}\text { Chapter } 4 & \text { A new algorithm for the automatic recognition of the } & \text { p. } 59\end{array}$ common carotid artery in longitudinal ultrasound scans

$\begin{array}{lll}\text { Chapter } 5 & \text { Automatic estimation of common carotid artery } & \text { p. } 85\end{array}$

diameter, orientation and curvature

Chapter $6 \quad$ Localization of common carotid artery intimal and p. 95 adventitial layers by means of ultrasound

$\begin{array}{lll}\text { Chapter } 7 & \text { General discussion } & \text { p. } 123\end{array}$

$\begin{array}{ll}\text { Summary } & \text { p. } 135\end{array}$

$\begin{array}{ll}\text { Samenvatting } & \text { p. } 138\end{array}$

$\begin{array}{ll}\text { Sommario } & \text { p. } 141\end{array}$

$\begin{array}{ll}\text { Thanks to... } & \text { p. } 145\end{array}$

$\begin{array}{ll}\text { About the author } & \text { p. } 147\end{array}$

$\begin{array}{ll}\text { Publications } & \text { p. } 148\end{array}$

$\begin{array}{ll}\text { Color figures } & \text { p. } 149\end{array}$ 

Chapter 1

General introduction 


\section{1 - Cardiovascular disease, arteries, atherosclerosis and risk factors}

Cardiovascular disease (CVD) is the major cause of death in the world (World Health Organization 2007). During the last decades there has been a progressive change in the way CVD prevention and treatment are addressed, from what was previously considered as mainly a heart-related disease. This led to the awareness that CVD is not just a synonym of coronary heart disease, but is related to a more general condition regarding the cardiovascular system as a whole, i.e. both the heart and the vascular tree (De Backer 2003).

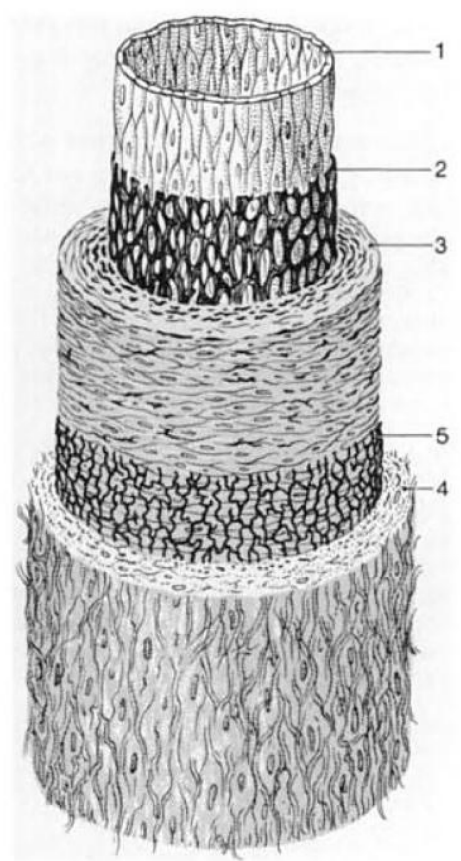

Fig. 1.1 - Layers composing the arterial wall: 1 - Tunica intima; 2 - Internal elastic lamina; 3 Tunica media; 4 - Tunica adventitia; 5 - External elastic lamina. Reproduced from Kahle (1993).

Arteries are blood vessels that transport blood from the heart to the target organs and tissues. They generally consist of three layers (Kahle 1993; Nichols 1998): a tunica intima, a tunica media and a tunica adventitia (Fig. 1.1). The intima is composed of a single layer of endothelial cells aligned with the long axis of the vessel, and serves the exchange of materials, fluids and gases between blood and tissue. It is separated from the media by the internal elastic lamina. The media is composed of layers of smooth muscle cells arranged along the circumferential direction, as well as elastin fibers. The muscular component of an artery actively balances forces exerted by blood pressure. The adventitia, separated from the media by the external elastic lamina, contains collagen and elastin fibers merging with the external 
connective tissue (Kahle 1993). Fig. 1.2 shows how vessel characteristics, i.e. lumen diameter, wall thickness, wall-to-diameter ratio and wall composition, change according to the segment considered in the vascular tree (Rushmer 1972).

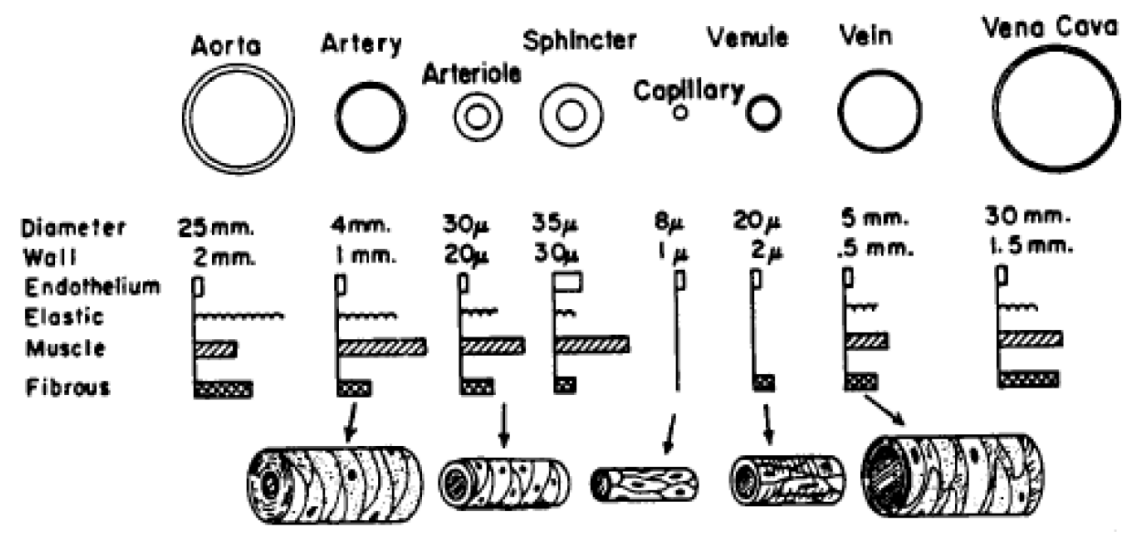

Fig. 1.2 - Composition and geometrical characteristics of vessel walls in the systemic circulation. Reproduced from Rushmer (1972).

The arterial diameter depends on the level of wall shear stress and tensile stress. In order to maintain the wall shear stress level at the endothelium constant, the artery diameter decreases in response to a decreased blood flow velocity, and vice-versa. Wall circumferential stress $\left(\sigma_{c}\right)$ is determined as $\sigma_{c}=P R / h$ in accordance with the Laplace law (Glagov 1995; Nichols 1998), where $P$ is the blood pressure, $R$ is the inner artery radius, and $\mathrm{h}$ is the wall thickness. In mammals, artery wall thickness adapts in order to maintain the circumferential stress at baseline level. When an artery obtains a larger diameter in response to chronically increased flow, the media physiologically adjusts its thickness accordingly. The intima may also participate in physiological wall adaptations (Glagov 1995).

Atherosclerosis is a chronic inflammatory process of the arteries, involving lipid uptake in the tunica intima and macrophage accumulation (Maton 1993), which can develop into arterial lesions, calcification and endothelial dysfunction (Fig. 1.3). Atherosclerosis is a form of arteriosclerosis, a term which refers more to general stiffening of arteries. Advanced atherosclerotic lesions are called "plaques", and in the carotid artery they are defined as (Stein 2008) "the presence of focal wall thickening that is at least $50 \%$ greater than that of the surrounding vessel wall or as a focal region with an intima-media thickness (IMT) greater than $1.5 \mathrm{~mm}$ that protrudes into the lumen and that is distinct from the adjacent boundary". The Fourth 
Task Force of the European Society of Cardiology (Graham 2007) clarified that the priorities for CVD prevention in clinical practice are:

1. patients with established atherosclerotic CVD;

2. asymptomatic individuals with increased risk of CVD;

3. close relatives of subjects with premature CVD or those at particularly high risk.

Prevention consists in changing modifiable behavioral and environmental habits (change in life style) for low risk subjects, whereas for high risk subjects cardioprotective drug treatment should be considered. Conventional risk factors for atherosclerotic CVD are smoking, blood pressure, blood cholesterol and glucose. More specifically, risk factors include hypertension, diabetes, obesity, increased total cholesterol, increased low-density lipoprotein (LDL) cholesterol, decreased highdensity lipoprotein (HDL) cholesterol, a positive family history, increased fibrinogen and C-reactive protein (CRP), advanced age, and physical inactivity (Graham 2007). Systolic and pulse pressure are known to be independent risk factors (Safar 2003).

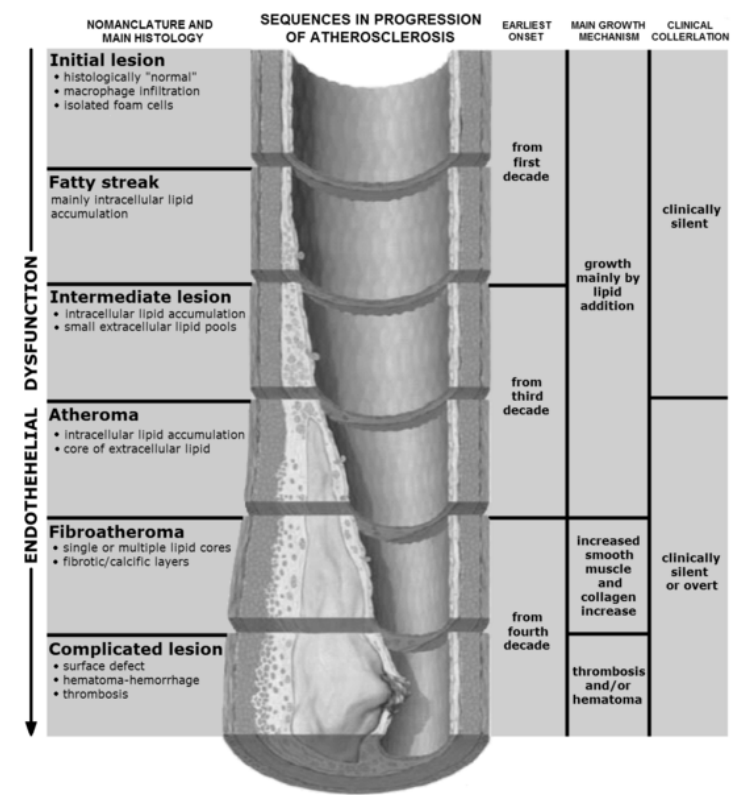

Fig. 1.3 - Progression of atherosclerosis in time. Reproduced from Wikipedia (GNU free documentation license). The figure emphasizes intimal dysfunction, not showing the increase in arterial diameter in response to chronically increased flow (and the consequent medial thickening) that can occur before intimal thickening.

CVD often causes premature abrupt death in affected patients. CVD-related events can be fatal due to their likeliness to appear abruptly without acute symptoms pre- 
ceding them, and therefore at moments when the subject is not hospitalized. The main life-threatening events associated with CVD are heart failure, myocardial infarction and stroke. Heart failure can be the consequence of a diseased and stiff arterial tree, transferring too much load on the heart itself and yielding consequent remodeling of the ventricles (e.g. hypertrophy). Myocardial infarction is usually related to myocardium ischemia. Stroke is mostly caused by hypertension in combination with atherosclerosis, because atherosclerotic plaques may rupture and generate emboli reaching the cerebral circulation.

The carotid artery (Fig. 1.4) is responsible for the supply of blood to the head. Two carotid arteries are present, one on each side of the neck. The carotid is composed of four main segments (Fig. 1.4): common carotid artery (CCA), carotid bifurcation, external carotid artery (ECA) and internal carotid artery (ICA) (Kahle 1993). The CCA passes through the neck without branching, and runs along the internal jugular vein and the vagus nerve. It then bifurcates into ICA and ECA, and at the level of the bifurcation the wall dilates into a structure (known as carotid bulb or carotid sinus) containing receptors for blood pressure (baroreceptors) and for partial oxygen pressure (chemoreceptors). The CCA can be considered as a large elastic artery (as the aorta) in the sense that the muscular part of the wall is not predominant (as opposed to more peripheral arteries) and the wall thickness is small with respect to the lumen diameter (Fig. 1.2). The combined thickness of the intimal and medial layers is called "intima-media thickness" (IMT). The diameter of the CCA can be considered from adventitia to adventitia (external diameter) or from intima to intima (internal diameter), as shown in Fig. 1.4.

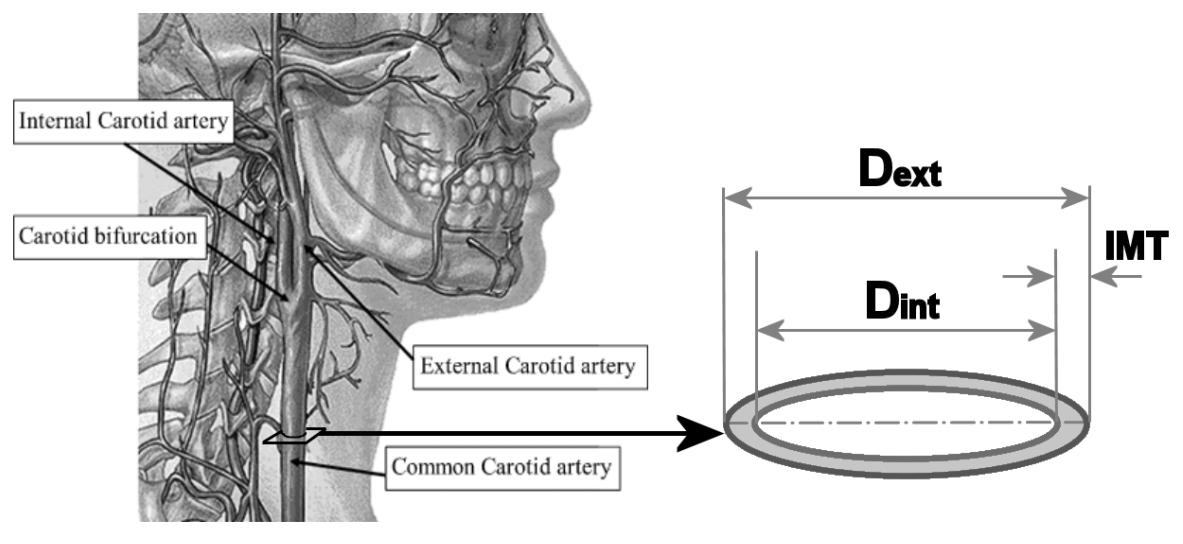

Fig. 1.4 - Anatomical locations of carotid artery segments (adapted from www.carolinavascular.com). The magnified section depicts the wall of the common carotid artery. The external diameter $\left(D_{\text {ext }}\right)$ is the adventitia-adventitia distance, whereas the internal diameter $\left(D_{\text {int }}\right)$ is the intima-intima distance. IMT stands for "intima-media thickness". 
Carotid IMT is commonly considered as a surrogate marker for atherosclerosis (Baldassarre 2000; De Groot 2004; Rajaram 2004), although a proof of causal relation between the two is lacking. Also elastic properties of arteries play a key role in the wellness of the arterial tree. Aging, essential hypertension and borderline hypertension induce losses in arterial elastic properties (Reneman 2005) and, therefore, in their capability to buffer and release blood during the heart cycle, thus augmenting systolic and pulse pressures that the heart has to generate in order to maintain a proper pump function.

CVD management for patients in clinics, in terms of both prevention and treatment, requires the ability for doctors and nurses to assess anatomical and dynamic wall properties in a non-invasive, fast, cost-effective, reliable and repeatable way. This is where ultrasonic imaging comes at hand (Reneman 2005; Stein 2008).

\section{2 - Non-invasive measurements of arterial properties by means of ultrasound}

A number of mechanical and anatomical parameters are in use to characterize the morphological and dynamic properties of the arterial tree. Non-invasive ultrasound is commonly used in clinics and research to estimate such parameters in population studies (Riley 1997; Cheng 2002; Reneman 2005; Mattace-Raso 2006) regarding epidemiology, intervention and normal values, and addressing either the general population or specific subject groups (eg. hypertensives and diabetics).

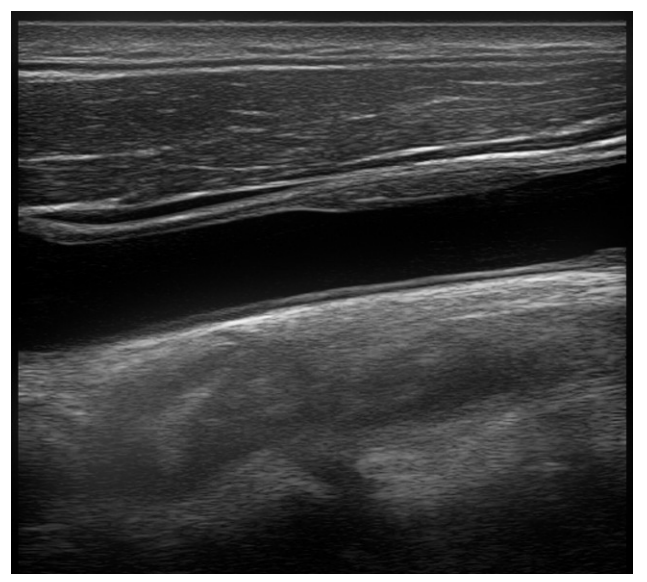

Fig. 1.5 - Longitudinal B-mode ultrasound scan of the common carotid artery. In this example, the upper wall ("near wall") of the artery presents irregularities, whereas on the lower wall ("far wall") the intima-media complex appears still uniform. 


\subsection{1 - Diameter, distension and strain}

Non-invasive assessment of the CCA diameter is commonly performed by means of B-mode ultrasound, imaging the artery in a longitudinal plane (Fig. 1.5). In these images, the lumen-intima boundaries of the artery walls appear as thin bright lines separated by a darker region (media) from the higher adventitia echoes. Tracking the diameter of an artery in time gives information about its structural properties (stiffness) as well as about its dynamical responses to changes in pressure and flow. The change in diameter occurring from the end diastolic to the systolic part of the beat (usually called distension) is related to the elastic properties of the arterial wall (Cheng 2002). Calling the diameter $D$ and the distension $\Delta D$, the circumferential strain of the artery can be calculated (assuming a thin wall) as $\triangle D / D$, whereas the radial strain is given by the relative change in wall thickness. Such quantities provide a measure of the relative deformations the arterial wall is exposed to (Reneman 2005).
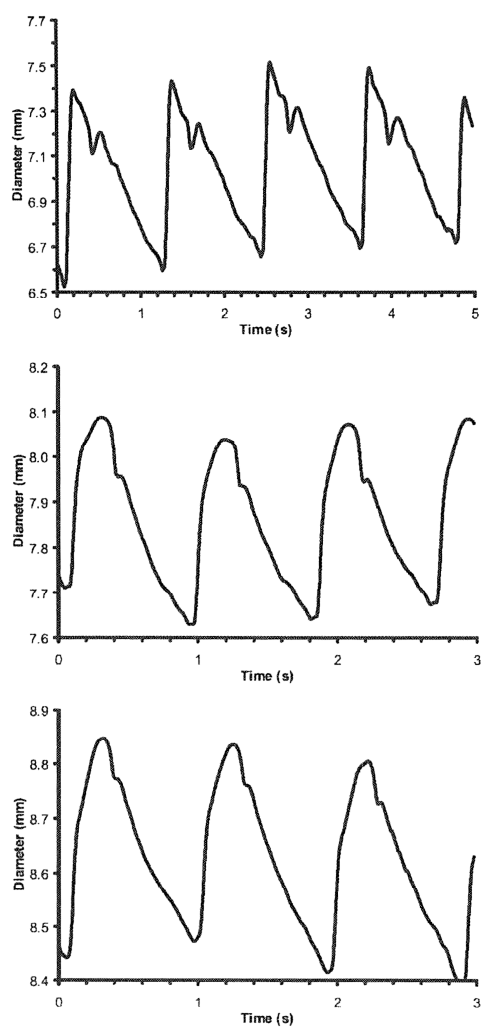

Fig. 1.6 - Examples of CCA diameter waveforms recorded using ultrasound. The distension decreases with age and hypertension. Top: young normotensive; middle: older normotensive; bottom: older hypertensive. Adapted from Reneman (2005). 
Aging and hypertension (Fig. 1.6) are among the major determinants of the characteristics of the CCA diameter waveform (Reneman 2005). The change in lumen diameter during the cardiac cycle primarily affects the intimal layer, while the adventitial layer is exposed to a lower radial strain and a lower circumferential strain (Segers 2004; Hoeks 2008). However, the CCA diameter is more commonly assessed on the media-adventitia transition due to its higher echogenicity. In addition to the CCA, non-invasive ultrasonic diameter assessment is also performed on femoral and brachial arteries. For the latter, flow-mediated dilation (FMD) is a popular technique to quantify the relation between blood flow velocity and diameter, testing brachial reactivity and endothelial function (Fan 2000).

\subsection{2 - Compliance, distensibility and stiffness}

Arterial compliance and distensibility are defined as, respectively, the absolute and relative change in arterial lumen volume due to a change in transmural pressure. The compliance reflects the ability of the arterial system to temporarily store blood volume, while the distensibility represents the mechanical load of the artery wall. Considering the fact that arteries are longitudinally tethered in vivo (Nichols 1998), their change in length during the cardiac cycle is negligible. Therefore, compliance and distensibility can also be evaluated per unit of length (compliance and distensibility coefficients $C C$ and $D C$ ), using the absolute and relative changes of artery cross-sectional area $A=2 \pi D$ rather than the segment volume (Meinders 2004; Reneman 2005).

$$
\begin{aligned}
& C C=\frac{\partial A}{\partial P} \\
& D C=\frac{\partial A}{A \partial P}
\end{aligned}
$$

In elastic arteries like the aorta and the carotid, the relationship between $A$ and $P$ is often approximated as linear during the cardiac cycle (Reneman 2005), and therefore the compliance and distensibility coefficients can be approximated by:

$$
\mathrm{CC}=\frac{\Delta \mathrm{A}}{\Delta \mathrm{P}}
$$




$$
D C=\frac{\Delta A}{A_{d} \Delta P}
$$

where $\Delta A$ and $\Delta P$ are the changes in cross-sectional area and pressure, respectively, from diastole to systole, and $A_{d}$ is the end diastolic cross-section.

Apart from measurement of arterial geometry and pressure, measures to determine arterial compliance, distensibility and stiffness can also be derived from pulse transit time and pulse waveform analysis (Pannier 2002; Boutouyrie 2008). High frame rate ultrasound imaging is used to determine the local pulse wave velocity (PVW) in the CCA (Brands 1998; Meinders 2001; Hermeling 2007). Arterial stiffness is also related to the augmentation index (AI), i.e. the ratio between the inflection-to-peak and the total amplitude of the pulse wave (Athanassios 2006).

\subsection{3 - Intima-media thickness}

The IMT (Fig. 1.4) of the CCA can be assessed by means of non-invasive B-mode ultrasound (Fig. 1.5), performed in a longitudinal section. To reduce estimation variation, multiple ultrasound scan lines are considered simultaneously to get an average value over a CCA segment spanning at least $1 \mathrm{~cm}$ (Stein 2008). It is advisable to distinguish the IMT of anterior and posterior CCA walls, because they have different echo properties (Touboul 2007). From an ultrasound image point of view, IMT is usually defined as the distance between the leading edges of the echoes generated by intima and adventitia (Wendelhag 1991; Wikstrand 1992), at least for the far wall. This takes into account the length of a single ultrasonic echo, which is primarily a function of system resolution and gain, rather than anatomy. For the near wall, the high echo of the adventitia tends to obscure the relatively low echo of the intima-lumen transition with consequently a lower reproducibility. The change in IMT (of the posterior wall) over time yields estimates of the radial strain of the CCA wall, which for a homogeneous wall should be similar (but opposite in sign) to the relative distension of the lumen.

\subsection{4 - Non-invasive pressure estimation}

The shape of the blood pressure waveform changes with the location in the arterial tree (Rowell 1968). Therefore, the pressure measured at the brachial artery with the standard cuff method differs from the one present at the carotid artery. Direct intravascular pressure measurements inside the CCA (Sugawara 2000) demonstrated the close similarity of the CCA pressure waveform and the diameter waveform ob- 
tained by means of echo-tracking. The pressure in the carotid artery, therefore, may be derived from non-invasive ultrasonic assessment of the diameter waveform (Van Bortel 2001), avoiding the problem associated with generalized transfer functions and modified phase delays (Meinders 2004). Methods proposed in literature to convert CCA distension waveforms to obtain pressure assume either a linear (Van Bortel 2001) or exponential relationship (Meinders 2004; Vermeersch 2008). In healthy subjects linear and exponential methods yield similar results (Vermeersch 2008; Zaccari 2008).

\subsection{5 - Blood flow and shear}

The velocity profile and the flow rate of blood can be measured non invasively in large arteries by means of Doppler ultrasound (Evans 2000). The velocity estimation is accurate close to the centerline of the vessel, whereas near the arterial walls artifacts arise in the velocity profile estimation due to interference of wall movements (Hoeks 2005). The evaluation of the velocity profile close to the walls is, however, highly relevant because it allows estimation of wall shear rate and wall shear stress (Brands 1995). Shear stress is known to be an important determinant of endothelial function and lumen diameter (Glagov 1995; Reneman 2006), and regions with abnormally low or oscillatory shear stress in the carotid artery are known to be prone to develop atherosclerotic plaques (Gnasso 1997; Boon 2009). Sensing and transduction of the wall shear stress on the arterial wall is in part mediated by the glycocalyx matrix (Reneman 2009).

\section{3 - Aim and outline of this thesis}

Ultrasound imaging is characterized by a highly user-oriented approach. Different sonographers do apply different image acquisition settings, modifying not only focal point and center frequency, but also parameters related to the subjective image perception, e.g. dynamic range and amplitude compression. The versatility of echo systems is a blessing, but also one of the major difficulties to interpret and correlate outcomes of large observational studies and/or clinical trials involving multiple sonographers, ultrasound systems and/or centres. To ensure clinical relevance of studies, standardization of procedures is strongly recommended (De Groot 2004; Lorenz 2007). Operational aspects that need to be continuously verified by the sonographer during non-invasive arterial US measurements are:

- perpendicularity between the US beam and the structures to be visualized (Touboul 2007); 
- measurement of IMT preferentially on the far wall; if taken also from the near wall, the two measurements should be acquired and reported separately (Touboul 2007);

- focus settings, gain settings and dynamic range compression (Potter 2008).

To estimate CCA position, diameter and IMT from B-mode ultrasound scans, and to properly assess their dynamics in time, image segmentation is of paramount importance. Even though manual and semi-automatic methods for segmentation of vascular US images are widely used, they are extremely time-consuming compared to automatic methods, and much less suited for proper standardization. Considering the large number of mechanical and dynamic parameters that can be inferred from non-invasive estimates of CCA diameter and IMT, it is clear that poor precision and repeatability of such measured geometrical properties negatively affects the clinical value. Therefore, segmentation of the acquired ultrasound vascular scans has a large impact on the relevance of the outcomes and should be as objective and robust as possible, regardless of its real-time or off-line implementation.

The aim of this thesis is to introduce novel algorithms for automatic segmentation of the common carotid artery in longitudinal ultrasound B-mode scans with a strong emphasis on minimization of sonographer interference and improvement of feedback to the sonographer. They are based on the analysis of ultrasonic echo amplitude signals retrieved directly from radio frequency (RF) raw ultrasound data. All analyses are applied to images obtained at a low frame update rate of $30 \mathrm{~Hz}$, irrespective of the purpose (dimension, distension). Processing B-mode sequences to eventually obtain distension waveforms has the advantage of a low computational load and inherent stability as opposed to standard echo tracking techniques based on radio frequency phase analysis (Hoeks 1990; Brands 1999). Segmentation algorithms should be objective, suppress confounding factors, and allow portability on data sets across different laboratories, different ultrasound scanners or acquisition modes. Standardization of protocols for acquisition and processing of ultrasound images will contribute to the quality of non-invasive estimation of CCA morphological (e.g. dimension, shape) and dynamic (e.g. distension) properties. A brief outline of the thesis is hereby provided.

Chapter 2 introduces basic concepts of ultrasound physics and technology, as an introduction for the following chapters based on original publications. An in-depth analysis of the effects of non-linear ultrasound image presentation (logarithmic compression and signal saturation) on automatic CCA diameter estimation is provided in Chapter 3, where two different edge detection methods are compared (sustain-attack filter and derivative approach). Chapter 4 introduces a novel algorithm for the automatic recognition of the CCA in ultrasound images, based on 
decimation, template matching and clustering. It allows to perform subsequently fully automatic arterial segmentation without user interference. Chapter 5 describes a method for the estimation of local orientation and curvature along the CCA centerline, associated with a directional smoothing of the CCA walls for better precision in external diameter estimation. Chapter 6 introduces an automatic methodology for the combined estimation of internal and external CCA diameter waveforms, as well as IMT waveforms of both carotid walls. The algorithm, based on the sustain-attack filter and on the "multiscale anisotropic barycenter", iteratively searches for the lumen-intima transition within a small region of interest. It also provides an automatic evaluation of the CCA ultrasonic beam incidence. Discussion and conclusion of the thesis are presented in Chapter 7.

\section{References}

Athanassios DP, Lekakis J, Ikonomidis I, Stamatelopulos K, Aznaouridis K, Karatzis E, Papamichael C, Marlomihelakis N, Kaklamanis $\mathrm{P}$, Mavrikakis M. Pressure wave reflections, central blood pressure, and aortic stiffness in patients with Adamantiades -Bechet's disease. American Journal of Hypertension 2006; 19: 660-666.

Baldassarre D, Amato M, Bondioli A, Sirtori CR, Tremoli E. Carotid artery intima-media thickness measured by ultrasonography in normal clinical practice correlates well with atherosclerosis risk factors. Stroke 2000; 31: 2426-2430.

Boon RA, Horrevoets AJ. Key transcriptional regulators of the vasoprotective effects of shear stress. Hamostaseologie 2009; 29: 39-43.

Boutouyrie P, Laurent S, Briet M. Importance of arterial stiffness as cardiovascular risk factor for future developments of new types of drugs. Fundamental \& Clinical Pharmacology 2008; 22: 241-246.

Brands PJ, Hoeks APG, Hofstra L, Reneman RS. A noninvasive method to estimate wall shear rate using ultrasound. Ultrasound in Medicine \& Biology 1995; 21: 171-185.

Brands PJ, Willigers J, Ledoux LAF, Reneman RS, Hoeks APG. A noninvasive method to estimate pulse wave velocity in arteries locally by means of ultrasound. Ultrasound in Medicine \& Biology 1998; 24: 1325-1335.

Brands PJ, Hoeks APG, Willigers J, Willekes C, Reneman RS. An integrated system for the non-invasive assessment of vessel wall and hemodynamic properties of large arteries by means of ultrasound. European Journal of Ultrasound 1999; 9: 257-266.

Cheng K-S, Baker CR, Hamilton G, Hoeks APG, Selifalian AM. Arterial elastic properties and cardiovascular risk/event. European Journal of Vascular and Endovascular Surgery 2002; 24: 383-397.

De Backer G, Ambrosini E, Borch-Jonsen K, Brotons C, Cifkova R, Dallongeville J, Ebrahim S, Faegeman O, Graham I, Mancia G, Manger Cats V, Orth-Gomér K, Perk J, Pyorala K, Rodicio JL, Sans S, Sansoy V, Sechtem U, Silber S, Thomsen T, Wood D. European guidelines on cardiovascular disease prevention in clinical practice. Third Joint Task Force of European and other Societies on cardiovascular disease prevention in clinical practice (constituted by representatives of eight societies and by invited experts). European Journal of Cardiovascular Prevention and Rehabilitation 2003; 10: S1-S78. 
De Groot E, Hovingh K, Wiegman A, Duriez P, Smit AJ, Fruchart JC, Kastelein JP. Measurement of arterial wall thickness as a surrogate marker for atherosclerosis. Circulation 2004; 109: 33-38.

Evans DH, McDicken WN. Doppler ultrasound. Physics, instrumentation and signal processing. Wiley,2000.

Fan L, Santiago P, Jiang H, Herrington DM. Ultrasound measurement of brachial flow-mediated vasodilator response. IEEE Transactions On Medical Imaging 2000; 19: 621-631.

Glagov S, Bassiouny HS, Giddens DP, Zarins CK. Intimal thickening: morphogenesis, functional significance and detection. Journal of Vascular Investigation 1995; 1: 2-14.

Gnasso A, Irace C, Carallo C, De Franceschi MS, Motti C, Mattioli PL, Pujia A. In vivo association between low wall shear stress and plaque in subjects with a symmetrical carotid atherosclerosis. Stroke 1997; 28: 993-998.

Graham I, Atar D, Borch-Jonsen K, Boysen G, Burell G, Cifkova R, Dallongeville J, De Backer G, Ebrahim S, Gjelsvik B, Hermann-Lingen C, Hoes A, Humpries S, Knapton M, Perk J, Priori SG, Pyorala K, Reiner Z, Ruilope L, Sans-Mendez S, Scholte op Reimer W, Weissberg P, Wood D, Yarnell J, Zamorano JL. European guidelines on cardiovascular disease prevention in clinical pratice: executive summary. Fourth Joint Task Force of the European Society of Cardiology and other Society on cardiovascular disease prevention in clinical practice (constituted by representatives of nine societies and by invited experts). European Heart Journal 2007; 28: 2375-2414.

Hermeling E, Reesink KD, Reneman RS, Hoeks APG. Measurement of local pulse wave velocity: effects of signal processing on precision. Ultrasound in Medicine \& Biology 2007; 33: 774-781.

Hoeks APG, Brands PJ, Smeets FAM, Reneman RS. Assessment of the distensibility of superficial arteries. Ultrasound in Medicine \& Biology 1990; 16: 121-128.

Hoeks APG, Reneman RS, Do Doppler systems color arteries red? Ed: Kowalewski TA, Lekszycki T, Nowicki A, Stanczyk M; Blood flow modelling and diagnostics, (Polish Academy of Sciences, Warsaw, 2005).

Hoeks APG, Hermeling E, Reneman RS, Artery wall mechanics determined by means of ultrasound. Ed.:Yim PJ, Vascular Hemodynamics Rounds, (John Wiley and Sons, New York, 2008).

Kahle W, Leonhardt H, Platzer w, Circulatory system. Color atlas and textbook of human anatomy: internal organs, (Thieme, 1993).

Lorenz MW, Markus HS, Bots ML, Rosvall M, Sitzer M. Prediction of clinical cardiovascular events with carotid intima-media thickness: a systematic review and meta-analysis. Circulation 2007; 115: 459-467.

Maton A, Hopkins J, McLaughlin CW, Johnson S, Warner MQ, LaHart D, Wright JD. Human biology and health. Englewood Cliffs, New Jersey, USA: Prentice Hall,1993.

Mattace-Raso FUS, Van Der Cammen TJM, Hoffman A, Van Popele NM, Bos ML, Schalekamp MADH, Asmar R, Reneman RS, Hoeks APG, Breteler MMB, Witteman JCM. Arterial stiffness and risk of coronary heart disease and stroke - The Rotterdam study. Circulation 2006; 113: 656-663.

Meinders JM, Kornet L, Brands PJ, Hoeks APG. Assessment of local pulse wave velocity in arteries using 2D distension waveforms. Ultrasonic Imaging 2001; 23: 199-215.

Meinders JM, Hoeks APG. Simultaneous assessment of diameter and pressure waveforms in the carotid artery. Ultrasound in Medicine \& Biology 2004; 30: 147-154. 
Nichols WW, O'Rourke MF. McDonald's blood flow in arteries. Theoretical, experimental and clinical principles. Arnold,1998.

Pannier BM, Avolio AP, Hoeks APG, Mancia G, Takazawa K. Methods and devices for measuring arterial compliance in humans. American Journal of Hypertension 2002; 15: 743-753.

Potter K, Reed CJ, Green DJ, Hankey GJ, Arnolda LF. Ultrasound settings significantly alter lumen and wall thickness measurements. Cardiovascular Ultrasound 2008; 6: DOI: 10.1186/1476-7120-6-6.

Rajaram V, Pandhya S, Patel S, Meyer PM, Goldin M, Feinstein MJM, Neems R, Liebson PR, Fielder BM, Macioch JE, Feinstein SB. Role of surrogate markers in assessing patients with diabetes mellitus and the metabolic syndrome and in evaluating lipid-lowering therapy. American Journal of Cardiology 2004; 93 : $32-48$.

Reneman RS, Meinders JM, Hoeks APG. Non-invasive ultrasound in arterial wall dynamics in humans: what we have learned and what remains to be solved. European Heart Journal 2005; 26: 960-966.

Reneman RS, Arts T, Hoeks APG. Wall shear stress - an important determinant of endothelial cell function and structure - in the arterial system in vivo. Journal of Vascular Research 2006; 43: 251-269.

Reneman RS, Vink H, Hoeks APG. Wall shear stress revisited. Artery Research 2009; DOI: 10.1016/j.artres.2009.02.005.

Riley WA, Evans GW, Sharrett AR, Burke GL, Barnes RW. Variation of common carotid artery elasticity with intimal-medial thickness: the ARIC study. Ultrasound in Medicine \& Biology 1997; 23: 157-164.

Rowell LB, Brengelmann GL, Blackman JR, Bruce RA, Murray JA. Disparities between aortic and peripheral pulse pressure induced by up right exercise and vasomotor changes in man. Circulation 1968; 37: $954-$ 964.

Rushmer RF. Organ physiology: structure and function of the cardiovascular system. Philadelphia: Saunders, 1972.

Safar ME, Levy B, Struijker Boudier HA. Current perspectives on arterial stiffness and pulse pressure in hypertension and cardiovascular diseases. Circulation 2003; 107: 2864-2869.

Segers P, Rabben SI, De Backer J, De Sutter J, Gillebert TC, Van Bortel L, Verdonck P. Functional analysis of the common carotid artery: relative distension differences over the vessel wall measured in vivo. Journal of Hypertension 2004; 22: 973-981.

Stein JH, Korkarz CE, Hurst T, Lonn E, Kendall CB, Mohler ER, Najjar S, Rembold C, Post W. ASE Consensus Statement. Use of carotid ultrasound to identify subclinical vascular disease and evaluate cardiovascular disease risk: a consensus statement from the American Society of Echocardiography carotid intima-media thickness Task Force endorsed by the Society for Vascular Medicine. Journal of the American Society of Echocardiography 2008; 21: 93-111.

Sugawara M, Niki K, Furuhata H, Ohnishi S, Suzuki S. Relationship between the pressure and diameter of the carotid artery in humans. Heart Vessels 2000; 15: 49-51.

Touboul P-J, Hennerici MG, Meairs S, Adams H, Amarenco P, Bornstein N, Csiba L, Desvarieux M, Ebrahim S, Fatar M, Hernandez Hernandez R, Jaff M, Kowantor S, Prati P, Rundek T, Sitzer M, Schminke U, Cardif JC, Taylor A, Vicaut E, Woo KS, Zannad F, Zureik M. Mannheim carotid intima-media thickness consensus (2004-2006). Cerebrovascular Diseases 2007; 23: 75-80. 
Van Bortel L, Balkestein EJ, Van der Heijden-Spek JJ, Vanmolkot FH, Staessen JA, Kragten JA, Vredeveld JW, Safar ME, Struijker Boudier HA, Hoeks APG. Non-invasive assessment of local pulse pressure: comparison of applanation tonometry and echo tracking. Journal of Hypertension 2001; 19: 1037-1044.

Vermeersch SJ, Rietzschel ER, De Buyzere ML, De Bacquer D, De Backer G, Van Bortel LM, Gillebert TC, Verdonck P.R., Segers P. Determining carotid artery pressure from scaled diameter waveforms: comparison and validation of calibration techniques in 2026 subjects. Physiological Measurement 2008; 29: 12671280.

Wendelhag I, Gustvsson T, Suurküla M, Berglund G, Wikstrand J. Ultrasound measurement of wall thickness in the carotid artery: fundamental principles and description of a computerized analysing system. Clinical Physiology 1991; 11: 565-577.

Wikstrand J, Wiklund O. Frontiers in cardiovascular science. Quantitative measurement of atherosclerotic manifestations in humans. Arteriosclerosis, Thrombosis and Vascular Biology 1992; 12: 144-119.

Zaccari I, Rossi AC, Bosboom EMH, Brands PJ. Pressure waveform estimation in the common carotid artery - Different methods in comparison. 2008. IFMBE Proceedings - 4th European Conference of the International Federation for Medical and Biological Engineering, (Antwerp, Belgium, 23-27 November 2008). 22: 2536-2539. Springer. 

Chapter 2

Medical ultrasound: physics and

technology 


\section{1 - Acoustic waves}

Sound is a mechanical vibration arising from the application of a time-varying stress to a medium. The vibration propagates through the medium in the form of acoustic waves, whose propagation velocity $c$ depends on medium properties. The term "ultrasound" refers to wave frequencies higher than the human auditory limit, usually taken to be $20 \mathrm{KHz}$.
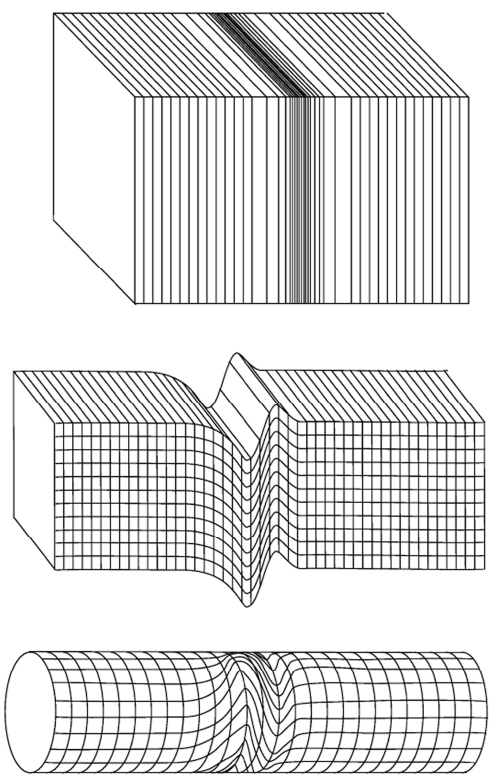

Fig. 2.1 - Different types of acoustic waves. From top to bottom: longitudinal, shear and torsional waves. Reproduced from Leighton (2007).

There is no net displacement of material particles during sound wave propagation, only acoustic energy is transferred. Each particle oscillates around its equilibrium position, and the direction of particle oscillation relative to the direction of wave propagation is what discriminates types of acoustic wave types (Fig. 2.1): longitudinal (compressional), shear (transverse), and torsional waves (Leighton 2007). The most common form of waves applied in medical ultrasound is the longitudinal form, consisting of alternating regions of compression and rarefaction due to the fact that directions of wave propagation and particle motion coincide. On the other hand, in shear and torsional waves both directions are perpendicular with respect to each other.

The velocity of propagation $c$ for longitudinal waves in gases and liquids is given by 


$$
c=\sqrt{\frac{K}{\rho}}
$$

where $K$ is the adiabatic bulk (elastic) modulus of the medium and $\rho$ is its mean density (Wells 1969; Cobbold 2007). $K$ is the inverse of the adiabatic compressibility $\kappa(K=1 / \kappa)$. In solids, velocities of propagation depend on the type of wave being propagated, and involve both the axial modulus and the shear modulus. This is because the shear rigidity of a solid couples some of the energy of a longitudinal wave into a transverse mode (Wells 1969), and vice-versa. In liquids, on the contrary, longitudinal waves are predominant.
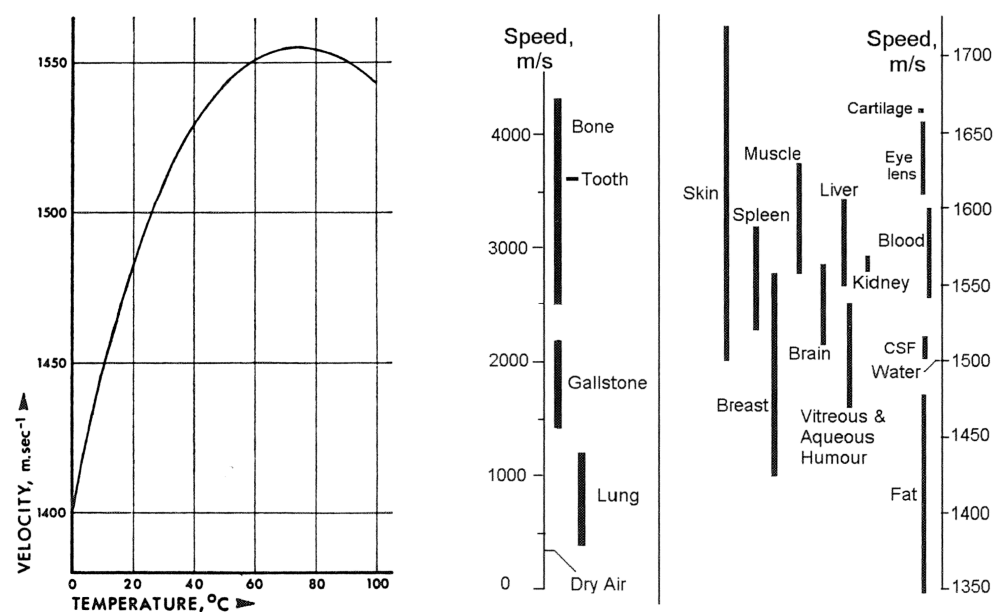

Fig. 2.2 - Left: variation of sound propagation velocity with temperature in distilled water, reproduced from Wells (1969); Right: speed of sound measurements in various biological media, reproduced from Cobbold (2007).

Soft biological tissues are composed mainly of water, therefore their sound propagation velocity can be considered similar to the one in liquids. At room temperature the acoustic propagation speed in distilled water is $1490 \mathrm{~m} / \mathrm{s}$ (Fig. 2.2), whereas in soft biological tissue $\left(36^{\circ} \mathrm{C}\right.$ ) it is in the order of $1540 \mathrm{~m} / \mathrm{s}$ (Wells 1969; Jensen 2007). Naturally this is an approximation because, as shown in Fig. 2.2, soft tissue is heterogonous and can be composed of skin, fat, muscle, blood, blood vessel walls and connective tissue, each one having a specific sound propagation velocity (Cobbold 2007).

The frequency of a harmonic ultrasound wave is directly related to the wavelength $\lambda$ and to the propagation speed $c$ : 


$$
f=\frac{c}{\lambda}
$$

It is of importance to note that a higher frequency results in a shorter wavelength and, hence, in a better resolution (see below). For $6 \mathrm{MHz}$ the wavelength will be $0.256 \mathrm{~mm}$ in biological tissues with an acoustic propagation speed of $1540 \mathrm{~m} / \mathrm{s}$.

\section{2 - Acoustic impedance, reflection and transmission}

For plane waves in a non-absorbing medium,

$$
\mathrm{p}=\mathrm{Z} \cdot \mathrm{v}
$$

where $P$ is the pressure and $v$ is the velocity of the particles in the medium. The characteristic acoustic impedance $Z$ of a medium (Wells 1969) is given by:

$$
Z=\rho \cdot c
$$

Its unit of measurement is the Rayl, i.e. $\mathrm{kg} /\left(\mathrm{m}^{2} \mathrm{~s}\right)$. When ultrasonic waves hit an interface between two media with acoustic impedances $Z_{1}$ and $Z_{2}$, their impedance ratio determines relative amounts of reflected and transmitted acoustic pressure. For a perpendicular incidence of a compressional wave on the reflecting surface, the relationships between reflected pressure $\left(p_{r}\right)$, transmitted pressure $\left(p_{t}\right)$ and incident pressure $\left(p_{i}\right)$ are (Wells 1969):

$$
\begin{aligned}
& \frac{p_{r}}{p_{i}}=\frac{z_{2}-z_{1}}{z_{2}+z_{1}} \\
& \frac{p_{t}}{p_{i}}=\frac{2 z_{2}}{z_{2}+z_{1}}
\end{aligned}
$$

If $Z_{1}=Z_{2}$ no reflection occurs. If $Z_{1}<Z_{2}$ the reflected wave is in phase with the incident one, whereas if $Z_{1}>Z_{2}$ the phase of the reflected wave differs $\pi$ radians from the phase of the incident wave, i.e. incident and reflected waves have reversed polarities (Wells 1969). 
In case the incident wave hits the reflecting interface with an angle $\theta_{i}$ with respect to the perpendicular direction, and if its wavelength is much smaller than the dimensions of the reflecting object, geometrical laws of reflection apply (Fig. 2.3).

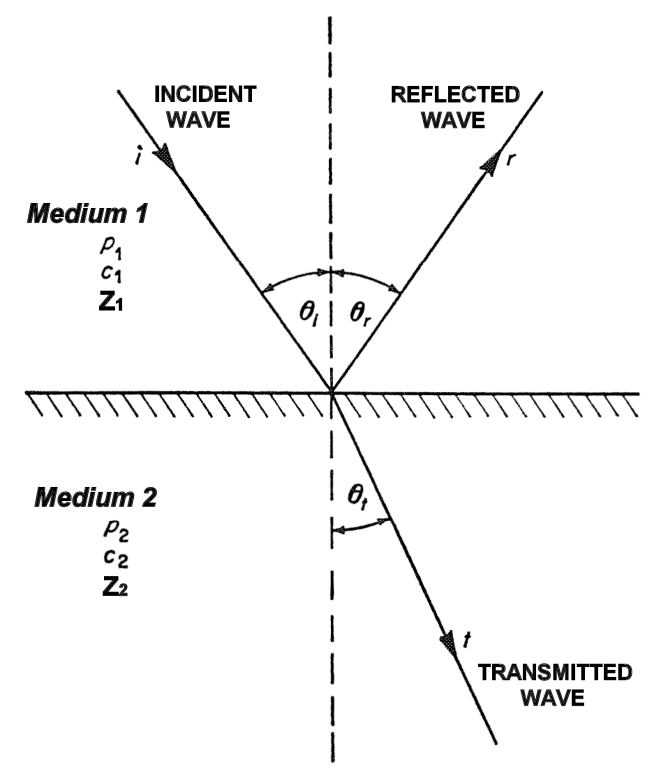

Fig. 2.3 - Incident, reflected and transmitted ultrasonic waves at a plane interface between two media with acoustic impedances $Z_{1}$ and $Z_{2}$, adapted from Wells (1969). In this case, the wavelength is supposed to be small compared to the dimension of the reflecting object.

The incidence angle $\theta_{i}$ and the reflection angle $\theta_{r}$ are equal. Transmission in the second medium occurs at an angle given by Snell's law (Wells 1969):

$$
\frac{\sin \theta_{i}}{\sin \theta_{t}}=\frac{c_{1}}{c_{2}}
$$

In this case, the relations between incident, reflected and transmitted pressures are:

$$
\begin{aligned}
& \frac{p_{r}}{p_{i}}=\frac{z_{2} \cos \theta_{i}-z_{1} \cos \theta_{t}}{Z_{2} \cos \theta_{i}+z_{1} \cos \theta_{t}} \\
& \frac{p_{t}}{p_{i}}=\frac{2 z_{2} \cos \theta_{i}}{z_{2} \cos \theta_{i}+z_{1} \cos \theta_{t}}
\end{aligned}
$$


Posing $\theta_{i}$ and $\theta_{t}$ equal to 0 converts equations (2.8) and (2.9) to the case of perpendicular incidence.

A particular case is encountered when $c_{2}$ is equal or larger than $c_{1}$, and $\theta_{i}=\sin ^{-1}\left(c_{1} / c_{2}\right)$. In that case, it follows from Snell's law that $\theta_{t}$ is equal to 90 degrees and, therefore, the transmitted wave propagates along the surface (Cobbold 2007). $\theta_{i}$ is then called critical angle, $\theta_{c}$.

If the dimensions of the object are comparable with or smaller than the wavelength of the ultrasonic wave, reflection is not only specular but also involves scattering (Wells 1969; Cobbold 2007). Scattered radiation reflects in an omnidirectional fashion, as opposed to specular reflection. For decreasing dimensions of the reflecting object (or of small irregularities on its surface) with respect to the ultrasound wavelength, the reflection pattern will attain a more omnidirectional shape (spherical pattern).

\section{3 - Attenuation of ultrasound}

As already mentioned, particle velocity and local pressure oscillate periodically in the medium supporting the propagation of a sound wave. Considering a harmonic oscillation involving a single frequency, the instantaneous velocity of the particles can be expressed as (Wells 1969):

$$
\mathrm{v}=\mathrm{v}_{0} \cos (\omega \mathrm{t})
$$

where $v_{0}$ is the maximum velocity amplitude, and $\omega=2 \pi f$ is the angular frequency. The oscillating pressure can therefore be expressed as

$$
P=\rho c v=\rho c v_{0} \cos (\omega t)=P_{0} \cos (\omega t)
$$

The intensity I of a sound wave, defined as the energy that passes through unit area in unit time (Wells 1969) and measured in Watts $/ \mathrm{m}^{2}$, is

$$
\mathrm{I}=\frac{1}{2} \rho \mathrm{cv}_{0}^{2}=\frac{1}{2} \frac{\mathrm{P}_{0}^{2}}{\rho \mathrm{\rho c}}
$$

While traveling through a physical medium, part of the energy of a wave is lost or redistributed by different kinds of mechanisms (Humphrey 2007). Attenuation phe- 
nomena that decrease the ultrasound intensity are deviation from a parallel beam and scattering due to discontinuities in elastic properties, compressibility and density (Wells 1969; Cobbold 2007). Another cause of energy loss is absorption, causing the conversion of vibrational energy into heat (Cobbold 2007). Absorption mechanisms are caused mainly by elastic hysteresis, viscosity, relaxation and heat conduction (Wells 1969). Altogether, scattering and absorption cause the wave intensity to decrease exponentially with distance (Cobbold 2007; Humphrey 2007):

$$
I=I_{0} \exp (-2 \alpha(f) x)
$$

$\alpha(f)$ is called "attenuation coefficient". Soft tissues typically present a frequencydependent attenuation, ranging from 0.5 to $1 \mathrm{~dB}$ per $\mathrm{MHz}$ per $\mathrm{cm}$, while blood attenuates only $0.2 \mathrm{~dB}$ per $\mathrm{MHz}$ per cm (Jensen 2007; Ali 2008). Therefore, the penetration depth attainable in the body strongly depends on the applied ultrasonic frequency, because a higher frequency results in a faster attenuation of sound. An ultrasound system will be able to image tissues up to a depth of $300-400 \lambda$ before the electronic noise starts to dominate in the received ultrasound signal. Frequencies used in medical applications of transcutaneous ultrasound usually range between $2 \mathrm{MHz}$ and $20 \mathrm{MHz}$ (Hoskins 2007). To compensate for attenuation, depthdependent amplification is applied on received ultrasound signals (TGC, i.e. time gain compensation). Of course, the TGC does not compensate for the decreased signal to noise ratio at greater depth.

\section{4 - Ultrasound imaging systems}

The main parts (Ali 2008) of an ultrasound imaging system (Fig 2.4) are front end (transmission, reception and digitization of ultrasound signals), beam forming (to focus along a scan line), mid end (with usually RF envelope detection and dynamic range compression) and back end (involving image enhancement and image rendering functions).

The probe (transmitting and receiving ultrasound) of an ultrasound scanner contains an array of piezoelectric ceramic elements (typically 128 or 192). The elements, acting as transducers, are selectively activated in order to interrogate subsequent lines of sights in the field of view, in a scanning fashion. There are several types of probes with different shapes (linear or curved, see Fig. 2.5) and involving different resonant frequencies of the piezoelectric crystals. Typical frequencies used are 3.5, 5, 7.5, 10 and $13 \mathrm{MHz}$. The desired imaging depth in the body for a specific applica- 
tion governs the choice of the center frequency of a probe, because higher frequencies imply less penetration depth.

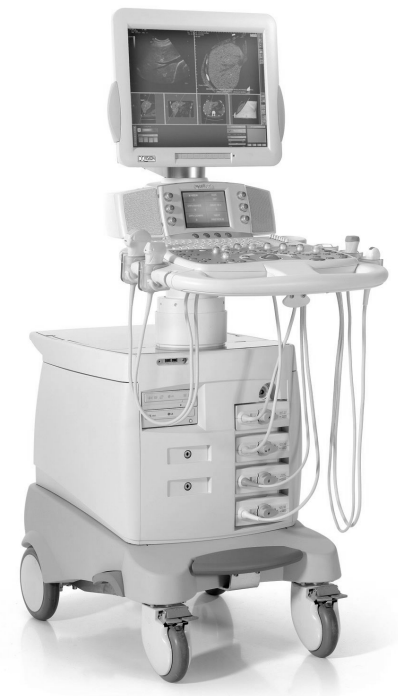

Fig. 2.4 - A commercial ultrasound scanner (Mylab 70 XVG, Esaote, Genova, Italy).
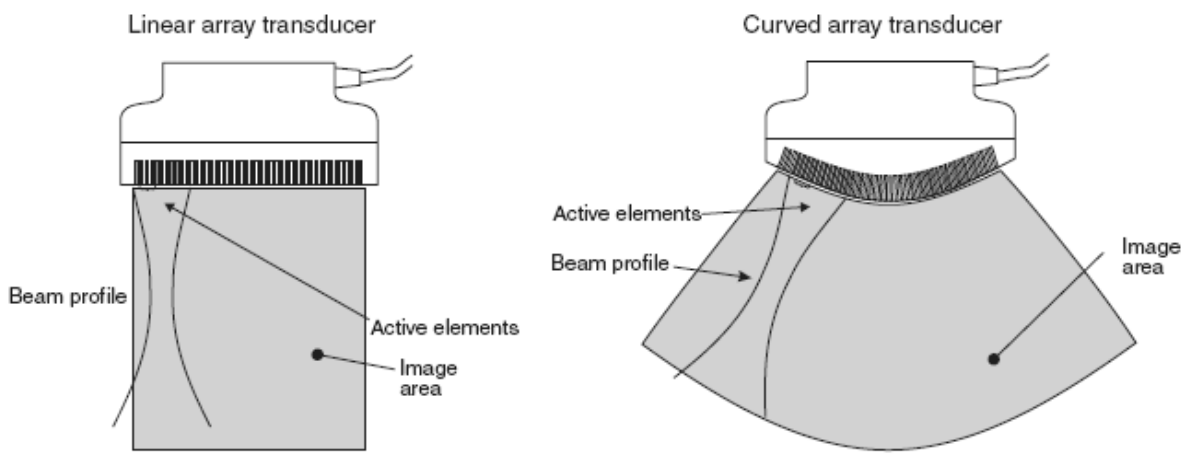

Fig. 2.5 - A linear array (left) and a curved array transducer (right). Reproduced from Jensen (2007).

Each transducer element is activated by a certain number (usually 1 to 2 ) of sinusoids, modulated with a bell-like function (usually a Gaussian) to transmit an ultrasound pulse. Since the time-bandwidth product is unitary (Gabor 1946; Hoeks 1991), a short emitted pulse can only be attained with a large transducer bandwidth, otherwise the pulse will be smoothed to some degree. In practice the relative transducer bandwidth is rather constant, i.e. the transducer bandwidth increases 
proportional with its center frequency. That is why for all high quality probes the emitted burst length is quite similar in terms of emitted periods independent of the emission frequency. Because the transducer acts not only as a transmitter, but also senses back the reflected echoes, image resolution will be affected by transmission bandwidth, path length through tissues with frequency-dependent attenuation and reception bandwidths. The resolution is different along the axial (along the beam) and lateral (across transducer elements) directions. The axial resolution of the system equals half the pulse length (Jensen 2007). Depending on wavelength and number of cycles in the activation pulse, the typical axial resolution of commercial ultrasound scanners ranges between 150 and $500 \mu \mathrm{m}$ and involves a trade-off between intended penetration depth and attainable axial resolution. The lateral resolution cell usually spans 3 times the probe pitch.

As shown in Fig.5, only a sub-section (called aperture) of the complete set of piezoelectric elements is activated to produce ultrasound waves. Focusing consists in delaying the excitations of the elements in the aperture in order for the wave front to converge at a desired point (the focal point). Beyond this point the beam will diverge, and therefore the beam width varies along depth. Focusing can also be achieved during reception (Jensen 2007); the depth-dependent arrival time permits a time-varying delay profile (dynamic focusing). The beamformer in an ultrasound scanner takes care of the transmission as well as the reception delays for each element in the aperture, and sums the received signals in order to obtain a radio frequency (RF) signal whose envelope represents a single line of sight in a B-mode (brightness mode) image. The RF signal is the basic component for signal processing in ultrasound scanners.

Due to both frequency-dependent attenuation (affecting pulse length as well) and beam width (determined by focusing within the plane of observation, and by an acoustic lens in perpendicular direction), the point spread function (PSF) of the system, representing the image response produced by a point scatterer at a particular location (Cobbold 2007), varies across image coordinates.

\section{5 - RF envelopes}

A B-Mode ultrasound image is a gray-scale representation of a matrix whose values are derived from the envelopes of the RF signals generated by the beamformer. The envelopes may be calculated by means of rectification and low pass filtering (smoothing), but this will induce a loss in resolution. To retain the bandwidth and thus resolution, envelopes can also be derived by calculating the magnitude of ana- 
lytic signals, obtained from RF signals by means of Hilbert Transform. Analytic signals are a combination of the original real signal with an imaginary signal that is a frequency-independent $-90^{\circ}$ phase shifted copy of the real signal. The easiest way to transform a real signal into an analytic signal is via the frequency domain. If $S(f)$ is the frequency spectrum of a real signal, then the corresponding analytic frequency spectrum $A(\mathrm{f})$ can be determined as follows:

$$
A(f)=\left\{\begin{array}{cc}
2 \cdot S(f) & f>0 \\
S(f) & f=0 \\
0 & f<0
\end{array}\right.
$$

As indicated in equation 2.14, the analytic frequency spectrum is single sided (only positive frequencies). For ultrasound RF-signals the zero and low-frequency components can be ignored. Transformation of the frequency spectrum $A(f)$ to the time domain produces the analytic signal.

However, to speed up processing and also to avoid low frequency resolution in case calculation involves short segments of RF signals, the frequency-domain Hilbert Transform can better be substituted with its time-domain counterpart (Rabiner 1975; Brands 1997). The continuous time Hilbert Transform is defined as (Shanmugan 1988):

$$
\mathrm{X}_{\mathrm{H}}(\mathrm{t})=\frac{1}{\pi} \int_{-\infty}^{\infty} \frac{\mathrm{X}(\alpha)}{\mathrm{t}-\alpha} \mathrm{d} \alpha=\mathrm{X}(\mathrm{t}) * \frac{1}{\pi \cdot \mathrm{t}}
$$

where $X(t)$ is a wideband signal, for example an ultrasonic RF echo, and * represents convolution. This operation is the same as passing $X(\mathrm{t})$ through a "quadrature filter":

$$
H(f)= \begin{cases}-j & f>0 \\ +j & f<0\end{cases}
$$

The analytic signal based on $X(t)$ and its Hilbert Transform is then (Rabiner 1975; Shanmugan 1988):

$$
X_{A}(t)=X(t)+j \cdot X_{H}(t)
$$


Once the quadrature representation is given the instantaneous envelope and phase are given respectively by

$$
\begin{aligned}
& R_{X}(t)=\sqrt{X^{2}(t)+X_{H}^{2}(t)} \\
& \Phi_{X}(t)=\tan ^{-1}\left\{\frac{X_{H}(t)}{X(t)}\right\}
\end{aligned}
$$

The computation of the instantaneous envelope (and phase) of analytic signals is a straightforward procedure, which makes it attractive for the calculation of RF envelopes. In the discrete time domain ( $\mathrm{n}$ being the discrete time index) the complex analytic signal is in the form:

$$
X_{A}(n)=X(n)+j \cdot X_{H}(n)
$$

As in the continuous time domain, the Discrete Time Hilbert Transform $X_{H}(n)$ can be obtained from $X(\mathrm{n})$ (which in our case is one of the real RF signals) by filtering it with a digital filter having a frequency response $H\left(e^{j \omega}\right)$ defined as (Rabiner 1975)

$$
H\left(e^{j \omega}\right)=\left\{\begin{array}{cc}
-j & 0 \leq \omega<\pi \\
j & \pi \leq \omega<2 \pi
\end{array}\right.
$$

In this case the Discrete Fourier Transform (DFT) of $X_{H}(n)$, has the following properties: DFT[ $\left.X_{H}(n)\right]=2 \cdot \operatorname{DFT}[X(n)]$ in the interval $0<\omega<\pi$, and DFT $\left[X_{H}(n)\right]=0$ in the interval $\pi<\omega<2 \pi$ (Rabiner 1975). The impulse response of the filter in eq. (2.21) may be obtained as:

$$
h(n)=\frac{1}{2 \pi}\left(\int_{0}^{\pi}-j \cdot \exp (j \cdot \omega \cdot n) d \omega+\int_{\pi}^{2 \pi} j \cdot \exp (j \cdot \omega \cdot n) d \omega\right)
$$

and hence it can be expressed in two equivalent forms: 


$$
h(n)=\left\{\begin{array}{ccc}
\frac{1-\exp (j \cdot \pi \cdot n)}{\pi \cdot n} & n \neq 0 \\
0 & n=0
\end{array} \text { or } h(n)=\left\{\begin{array}{cc}
\frac{2 \sin ^{2}(\pi \cdot n / 2)}{\pi \cdot n} & n \neq 0 \\
0 & n=0
\end{array}\right.\right.
$$

Fig. 2.6 shows the frequency response and impulse response of the Hilbert Transform. It is clear that $h(n)$ is physically unrealizable since it is two-sided infinite in extent, and hence it has to be somehow approximated. Brands (1997) introduced an approximation for band-limited signals in order to perform the Discrete Hilbert Transform in the time domain:

$$
X_{A}(n)=X(n)+j \cdot \frac{2}{\pi} \sum_{m=1}^{H w} \frac{X(n+m)-X(n-m)}{m} \quad m=1,3,5, \ldots H_{w}
$$

$H_{w}$ is the length of one side of the spatial Hilbert transformation window, centered on the current sample of $X(n)$. The envelope of the RF signal is then computed as the magnitude of $X_{A}(n)$.

HILBERT TRANSFORMER

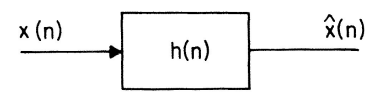

(a)

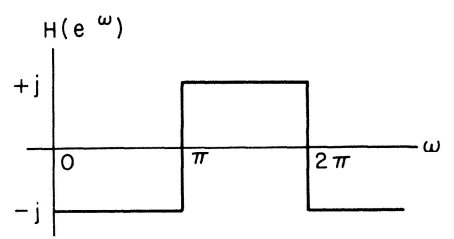

(b)

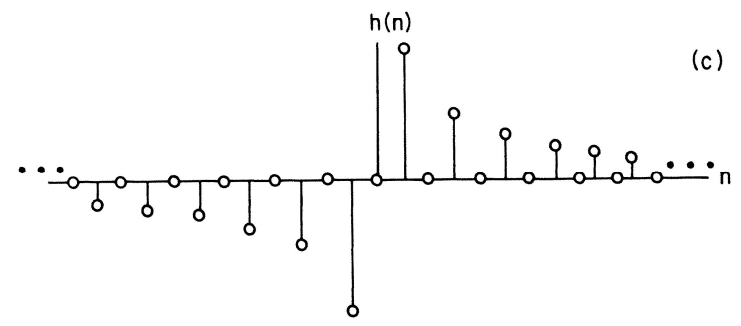

Fig. 2.6 - Schematic representation of an Hilbert transformer (a). Frequency response (b) and impulse response (c) of the Discrete Hilbert Transform. Reproduced from Rabiner (1975). 
Due to the introduced approximation, the computed envelope contains an error, which is a function of both the frequency content of the RF signal and of the oversampling factor (i.e., the ratio between the sampling frequency and the central frequency of the RF signal). The signals should always be band-pass filtered prior to the computation of the Hilbert Transform to prevent that any DC component (possibly introduced by amplifiers) interferes with the Hilbert Transform and, indirectly, with the envelope calculation.

It can be shown (Ledoux 1998) that because of the typical Gaussian amplitude distribution of RF signals the resulting amplitude distribution of the RF envelope has a Rayleigh distribution (Fig. 2.7). Because of the very wide dynamic range of RF signals (70 to $100 \mathrm{~dB}$ ) and their envelopes, logarithmic compression is often applied in commercial ultrasound scanners, allowing echo amplitudes adjusted to the lower dynamic range of monitors (Dutt 1996; Ali 2008). Compression changes the envelope statistics and the image appearance (Dutt 1995; Cobbold 2007).

A

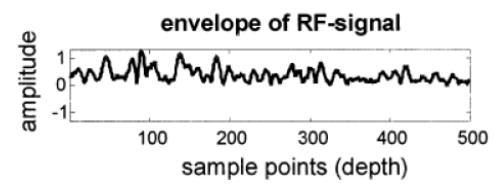

B

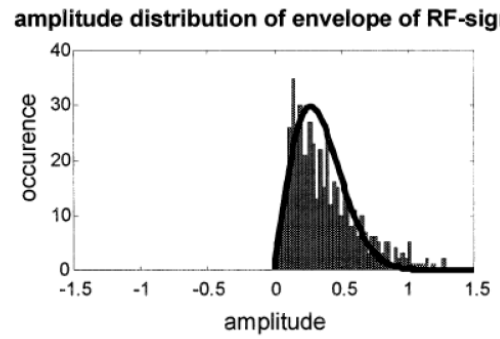

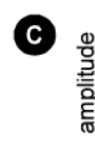

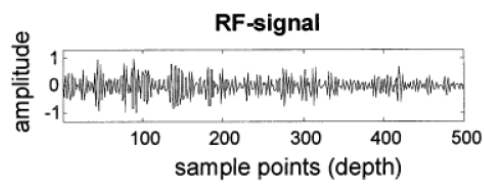

D

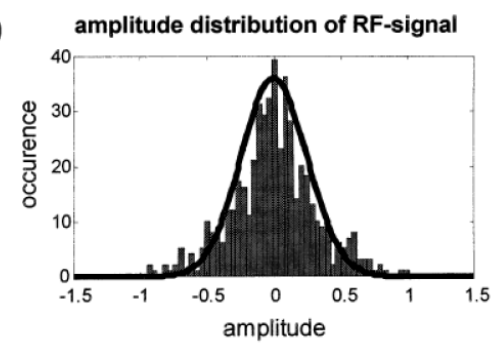

Fig. 2.7 - RF signals (c) have a Gaussian amplitude distribution (d), while their envelopes (a) have a Rayleigh amplitude distribution (b). Reproduced from Ledoux (1998).

\section{6 - Scan conversion}

The coordinate system for acquisition of RF signals and their envelopes vary with the type of probe used. Linear arrays acquire in Cartesian coordinates, whereas curved arrays acquire in polar coordinates (Figs 2.5 and 2.8). On the other hand, pixel grids used to present images on screen are always in Cartesian coordinates, and the pixel density depends on the screen resolution. The scan converter takes care of the interpolation of ultrasound envelope data (Fig. 2.9) into displayed data 
(Ali 2008), commonly via a bilinear interpolation. In high-end systems the pixel density is higher than for low-end systems.
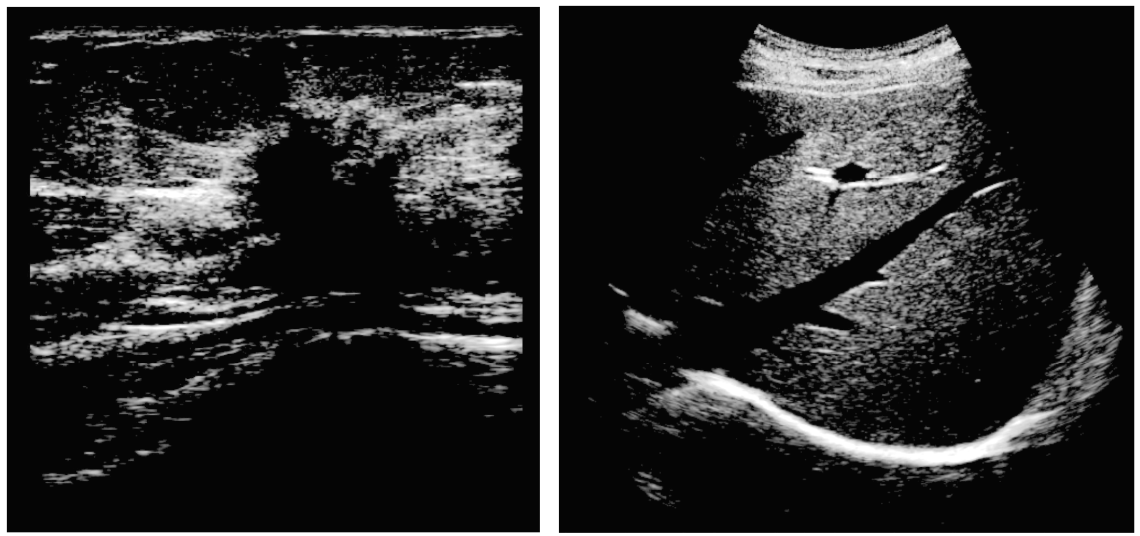

Fig. 2.8 - Left: a linear scan of the breast; Right: a convex scan of the liver.
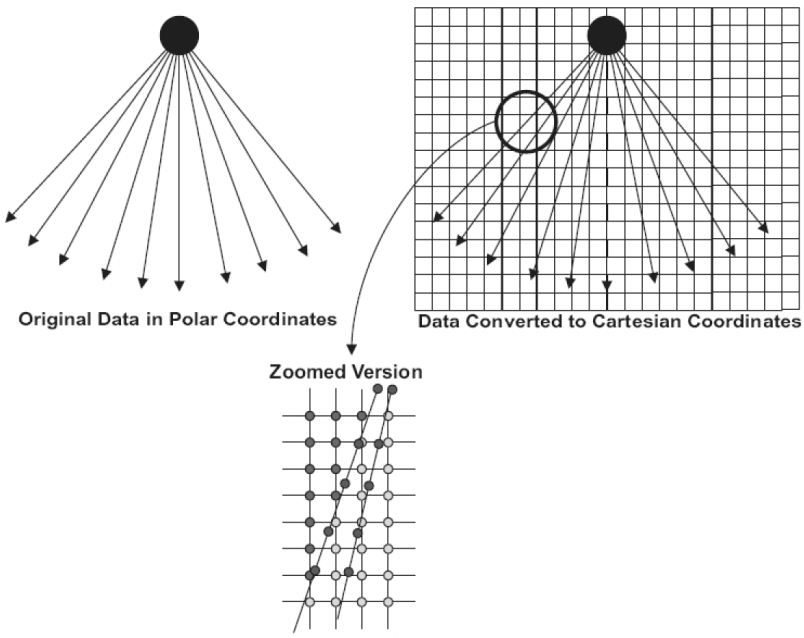

Fig. 2.9 - Scan conversion from polar to Cartesian coordinates. Adapted from Ali (2008).

\section{7 - Non-linear propagation and harmonic imaging}

During sound propagation in biological tissues, non-linear phenomena occur because sound velocity varies with instantaneous density (Jensen 2007). A higher acoustic pressure increases tissue density, while a lower pressure decreases it. Consequently the positive pressure part of an acoustic wave travels slightly faster than the negative pressure part, and hence a sinusoidal wave will gradually convert into a 
sawtooth shaped wave (Wells 1969). This conversion is accompanied with the production of higher harmonics. This phenomenon is exploited for enhancing image characteristics in what is called native harmonic imaging (Verbeek 2000; Cobbold 2007; Jensen 2007). Harmonic imaging can also involve imaging of air-filled contrast bubbles at their resonance frequency.

\section{8-Doppler ultrasound}

Velocities and flow rates of scatterers (such as red blood cells) can be measured non-invasively with ultrasound exploiting the Doppler effect (Hoeks 2005). It is based on the fact that the apparent frequency of a constant frequency source depends on both the motion of the source and that of the receiver (Wells 1969). When ultrasound is transmitted at a frequency $\mathrm{f}_{O}$ and is reflected by moving scatterers whose velocity has magnitude $u$ and is oriented at an angle $\phi$ with respect to the ultrasound beam, it is received with a different frequency $\mathrm{f}_{R}$ (Evans 2000). The difference between the received and transmitted frequencies is called Doppler shift $f_{D}$, and is given by (Cobbold 2007):

$$
f_{D}=f_{R}-f_{0}=-\frac{2 u}{c} f_{0} \cos (\varphi)
$$

The minus sign indicates that the Doppler shift is positive when the scatterers move towards the transducer, and negative when they move away from the transducer.

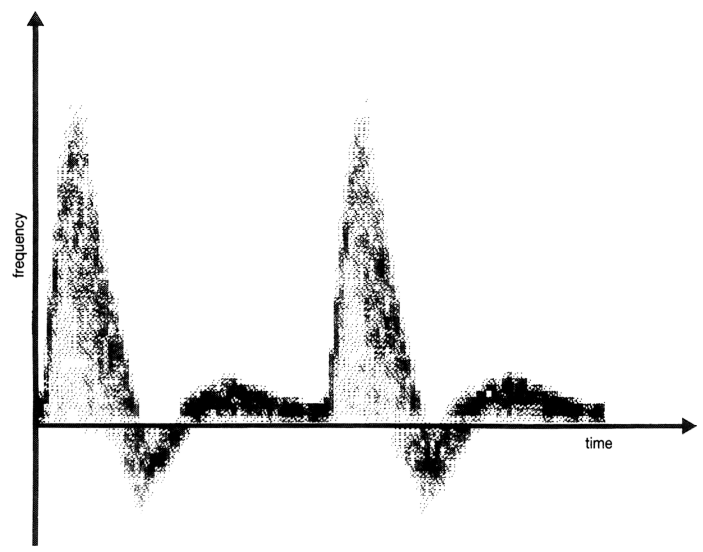

Fig. 2.10 - Sonogram of the Doppler shift from a femoral artery. Flow towards the transducer appears as a positive frequency, whereas flow away from the transducer is associated with a negative shift. Reproduced from Evans (2000). 
Doppler systems are broadly categorized in continuous wave (CW) systems, transmitting and receiving ultrasound waves continuously, and pulsed wave (PW) systems, emitting ultrasound pulses allowing selection of the depth at which movement is observed (Evans 2000). The Doppler signal can be encoded as an audio signal and visualized as a time dependent frequency distribution, i.e. sonogram (Fig. 2.10). Alternatively one may present the detected velocities as color-coded overlay on an ultrasound image.

\section{9 - Ultrasound image processing}

Ultrasound images have a typical grainy appearance due to coherent and random phase interference of the scattered waves within the resolution cell: the speckle pattern (Burckhardt 1978; Wagner 1983). Because speckle reduces resolution, contrast, and sharpness of the edges, it complicates image segmentation for further analysis. Various speckle reduction techniques have been proposed (Bamber 1986; Koo 1991; Loizou 2005; Michailovich 2006; Ali 2008), based on speckle modeling, local RF statistics, histogram equalization, averaging, anisotropic diffusion, wavelets and spatial compounding. Every technique has its own advantages and disadvantages with respect to image quality and computation time. When dealing with automatic boundary delineation algorithms, though, pre-processing the image with a speckle reduction filter can highly affect the morphological outcome of the segmentation. Boundary detection in ultrasound images is commonly based on highpass filters, high-boost filters and derivative filters (Ali 2008). Also dynamic programming techniques are often used, such as snakes (Cheng 2002) and active contours (Tao 2003). Region-based techniques for ultrasound image segmentation can be based on statistical distributions (Destempres 2009; Slabaugh 2009) and on texture analysis (Valckx 1997; Coolen 1999).

\subsection{0 - Arterial diameter tracking}

A common approach to track in time the diameter of an artery in transcutaneous ultrasound scans is based on a wall track algorithm. The latter relies on the analysis of the time-dependent phase behavior of RF signals segments returned by the arterial walls. What is directly estimated is the vessel wall velocity, by means of analytic cross-correlation models applied to specific RF estimation windows, placed manually or automatically on the vessel wall reflections (Brands 1999). Examples of frequency-domain cross-correlation models used are the CCM, a narrowband real model (De Jong 1990), and the C3M, based on complex signals and allowing wideband RF signals (Brands 1997) with lower estimation errors. Once the initial position 
of artery walls (and thus the initial arterial diameter) is manually or automatically established, the temporal change in diameter is assessed by means of cumulative integration of displacement increments of both arterial walls utilizing the phase of the RF signals (equation 2.19). The RF signal windows for cross-correlation are then displaced in time according to the calculated instantaneous wall position (Hoeks 1990).

The wall track algorithm presents a disadvantage: small errors in the wall velocity estimation will influence the diameter estimation in a cumulative way, making the method inherently prone to yield arterial diameter waveforms with a drift. Because of this complication, the wall track program should be either restricted to a recording duration of maximally 5-6 seconds (respiration cycle) or should be reset at each cardiac cycle. In this thesis arterial diameter detection methods based on the direct segmentation of B-mode ultrasound images will be proposed, rather than relying on indirect wall motion estimation. Segmentation-oriented approaches do not suffer from drift as each subsequent image is treated independently.

\section{References}

Ali M, Magee D, Dasgupta U. Signal processing overview of ultrasound systems for medical imaging. Texas Instruments, white paper, 2008.

Bamber JC, Daft C. Adaptive filtering for reduction of speckle in ultrasonic pulse-echo images. Ultrasonics 1986; 24: 41-44.

Brands PJ, Hoeks APG, Ledoux LAF, Reneman RS. A radio frequency domain complex cross-correlation model to estimate blood flow velocity and tissue motion by means of ultrasound. Ultrasound in Medicine \& Biology 1997; 23: 911-920.

Brands PJ, Hoeks APG, Willigers J, Willekes C, Reneman RS. An integrated system for the non-invasive assessment of vessel wall and hemodynamic properties of large arteries by means of ultrasound. European Journal of Ultrasound 1999; 9: 257-266.

Burckhardt CB. Speckle in ultrasound B-Mode scans. IEEE Transactions On Sonics And Ultrasonics 1978; 25:1-6.

Cheng D, Schmidt-Trucksass A, Cheng K, Burkhardt H. Using snakes to detect the intimal and adventitial layers of the common carotid artery wall in sonographic images. Computer Methods and Programs in Biomedicine 2002; 67: 27-37.

Cobbold RSC. Foundations of biomedical ultrasound. Oxford University Press, 2007.

Coolen J, Engelbrecht MR, Thijssen JM. Quantitative analysis of ultrasonic B-mode images. Ultrasonic Imaging 1999; 21: 157-172.

De Jong PGM, Arts T, Hoeks APG, Reneman RS. Determination of tissue motion velocity by correlation interpolation of pulsed ultrasonic echo signals. Ultrasonic Imaging 1990; 12: 84-98. 
Destempres F, Meunier J, Giroux M-F, Soulez G, Cloutier G. Segmentation in ultrasonic B-mode images of healthy carotid arteries using mixtures of Nakagami distributions and stochastic optimization. IEEE Transactions On Medical Imaging 2009; 28: 215-229.

Dutt V, Statistical analysis of ultrasound echo envelope. Mayo Graduate School, Rochester, (1995). PhD Thesis.

Dutt V, Greenleaf JF. Adaptive speckle reduction filter for log-compressed B-Scan images. IEEE Transactions On Medical Imaging 1996; 15: 802-813.

Evans DH, McDicken WN. Doppler ultrasound. Physics, instrumentation and signal processing. Wiley, 2000.

Gabor D. Theory of communication. Journal of The Institution of Electrical Engineers 1946; 93: 429-457.

Hoeks APG, Brands PJ, Smeets FAM, Reneman RS. Assessment of the distensibility of superficial arteries. Ultrasound in Medicine \& Biology 1990; 16: 121-128.

Hoeks APG, Hennerici MG, Reneman RS. Spectral composition of Doppler signals. Ultrasound in Medicine \& Biology 1991; 17: 751-760.

Hoeks APG, Reneman RS, Do Doppler systems color arteries red? In: Blood flow modelling and diagnostics, Polish Academy of Sciences, Warsaw, 2005.

Hoskins PR. Physical properties of tissues relevant to arterial ultrasound imaging and blood velocity measurement. Ultrasound in Medicine \& Biology 2007; 33: 1527-1539.

Humphrey VF. Ultrasound and matter - physical interactions. Progress in Biophysics and Molecular Biology 2007; 93: 195-211.

Jensen JA. Medical ultrasound imaging. Progress in Biophysics and Molecular Biology 2007; 93: 153-165.

Koo Jl, Park SB. Speckle reduction with edge preservation in medical ultrasonic images using an homogeneous region growing mean filter. Ultrasonic Imaging 1991; 13: 211-237.

Ledoux LAF, Willigers JM, Brands PJ, Hoeks APG. Experimental verification of the correlation behavior of analytic ultrasound radiofrequency signals received from moving structures. Ultrasound in Medicine \& Biology 1998; 24: 1383-1396.

Leighton TG. What is ultrasound? Progress in Biophysics and Molecular Biology 2007; 93: 3-83.

Loizou CP, Pattichis CS, Christodoulou Cl, Istepanian RSH, Pantzaris M, Nicolaides A. Comparative evaluation of despeckle filtering in ultrasound imaging of the carotid artery. IEEE Transactions On Ultrasonics, Ferroelectrics and Frequency Control 2005; 52: 1653-1669.

Michailovich OV, Tannenbaum A. Despeckeling of medical ultrasound images. IEEE Transactions On Ultrasonics, Ferroelectrics and Frequency Control 2006; 53: 64-78.

Rabiner LR, Gold B, Theory of discrete-time linear systems. In: Theory and application of digital signal processing, Prentice-Hall, Englewood Cliffs, 1975.

Shanmugan KS, Breipohl AM, Special classes of random processes. In: Random Signals - Detection, estimation and data analysis, John Wiley and Sons, 1988. 
Slabaugh G, Unal G, Wels M, Fang T, Rao B. Statistical region-based segmentation of ultrasound images. Ultrasound in Medicine \& Biology 2009; 35: 781-795.

Tao Z, Jaffe CC, Tagare HD, Tunnelling descent: a new algorithm for active contour segmentation of ultrasound images. In: Information Processing in Medical Imaging, Springer Berlin / Heidelberg, 2003.

Valckx FMJ, Thijssen JM. Characterization of echographic image texture by cooccurrence matrix parameters. Ultrasound in Medicine \& Biology 1997; 23: 559-571.

Verbeek X, Tissue perfusion assessment with ultrasound contrast agents. Universiteit Maastricht, Biophysica, Maastricht, (2000). Phd Thesis.

Wagner RF, Smith SW, Sandrick JM, Lopez H. Statistics of speckle in ultrasound B-scans. IEEE Transactions On Sonics And Ultrasonics 1983; 30: 156-163.

Wells PNT, Fundamental Physics. In: Physical principles of ultrasonic diagnosis, Academic Press, London, 1969. 



\section{CHAPTER 3}

\section{Effects of non-linear processing on common carotid artery diameter estimation}

The content of this chapter is based on the publication: Rossi AC, Brands PJ, Hoeks APG. Nonlinear processing in B-mode ultrasound affects carotid diameter assessment. Ultrasound in Medicine \& Biology 2009; 35: 736-747. 


\section{1 - Abstract}

Non-invasive diameter assessment in the common carotid artery (CCA) by means of ultrasound is a useful technique for estimation of arterial mechanical and dynamic properties, clinical screening and treatment monitoring. Prior to presentation on screen, ultrasound images are subjected to non-linear processing, e.g. logarithmic compression and noise-level thresholding, to improve visualization. In addition, signal saturation may occur, either in the received radio-frequency (RF) signals or in their envelopes. The objective of this study is to evaluate the effect of signal nonlinearities on CCA diameter measurements by means of non-invasive B-mode ultrasound, comparing the performance of two different edge detectors.

In 14 healthy subjects, 3 repeated ultrasonic acquisitions (6 seconds) without saturation were performed. The acquired RF signals were subjected off-line to envelope detection, logarithmic compression and various degrees of saturation applied to the signals prior or after envelope detection. For the purpose of CCA diameter estimation, artery walls were automatically outlined frame by frame. As automatic edge detectors we considered the sustain-attack filter (SAF), based on exponentially decaying reference functions, and a derivative approach (DER), relying on the positions of first derivative maxima. Both methods are applied within a region of interest located on the CCA. No regularization of the detected wall positions by means of pre- or post-processing is presently applied, in order to directly relate the outcome of the edge detectors to the applied non-linear processing.

Diameter values assessed with SAF are unaffected by logarithmic compression, due to the possibility to integrate the compression characteristic of the ultrasound system into the method. The estimated diameters values obtained with DER instead show differences in the order of $10 \%$ due to compression. Saturation affects DER more than SAF; DER exhibits larger intra-recording and intra-subject variations in the estimated diameter values. Therefore SAF gives more precise and robust CCA diameter estimates than DER does, and is more suited for integration in algorithms meant for vascular ultrasound image segmentation. This study demonstrates the relevant effects of non-linearities such as saturation and logarithmic compression on the quality of non-invasive US CCA diameter measurements.

\section{2 - Introduction}

Non-invasive assessment of arterial wall dynamics by means of ultrasound (US) in the common carotid artery (CCA), as well as in other arteries such as femoral and 
brachial artery, involves frame-by-frame identification of the vessel wall positions in order to track the arterial diameter over time (Meinders 2004; Reneman 2005). Ultrasonic diameter tracking is a fast, cost-effective and totally non-invasive procedure that can be used in clinics for assessing CCA distensibility (Mattace-Raso 2006) and brachial flow-mediated dilatation (Sonka 2002), both associated with cardiovascular risk. Reliable measures of CCA diameter are of paramount importance also for the subsequent assessment of carotid intima-media thickness (IMT), which is commonly considered as a surrogate marker for atherosclerosis (De Groot 2004). The age-dependent increases in CCA diameter are in the order of $100 \mu \mathrm{m}$ per decade (Samijo 1998). Therefore, consistent and reliable methodologies are strongly needed for CCA diameter assessment by means of ultrasound, in order to avoid that confounding factors, related to the operational settings of the US acquisitions, affect the outcome of the measurements and thus their clinical relevance.

Diameter estimation is commonly based on real-time or off-line image processing performed on longitudinal B-mode scans. The procedure strongly relies on automatic edge detection in order to consistently locate arterial walls, if necessary preceded by despeckling and followed by spatial and/or temporal post-processing. US B-mode images exhibit sharp transitions in echo amplitude level, indicating interfaces between tissues with varying acoustic impedance. When imaging a healthy CCA in a longitudinal insonation plane, two distinct interfaces can be seen at each wall (Fig. 3.1), one between blood and the "tunica intima", and one between the "tunica media" and the "tunica adventitia". This is because both blood and media are echolucent, whereas intima and adventitia are echogenic. This work focuses on the assessment of the external diameter of the CCA, based on the location of media-adventitia interfaces which have a much higher reflectivity than the lumenintima ones. The choice of the CCA as vessel of interest is governed by the "Mannheim Carotid Intima-Media Thickness Consensus" (Touboul 2007), which indicates the CCA as an optimal location for US acquisition as opposed to carotid bulb and internal carotid artery.

Various image-processing techniques have been proposed to perform semiautomatic segmentation of arterial US images, with highly varying levels of processing complexity. Due the nearly horizontal orientation of the CCA in B-mode frames, it is very common to locate its walls by means of grey-level gradient analysis along echo scan lines (Selzer 1994; Stadler 1997; Graf 1999; Stein 2005; Craiem 2007). Another possibility is given by active contours (Cheng 2002; Loizou 2006; Delsanto 2007), which take into account the two-dimensional gray-level gradient in the form of "external energy" for the contour. 


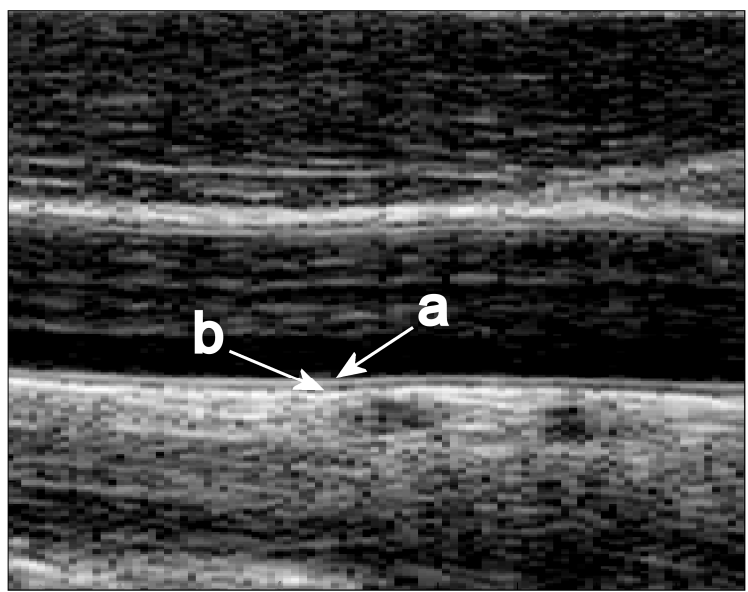

Fig. 3.1 - Longitudinal ultrasound scan of the common carotid artery as acquired using a commercial echograph. The lumen-to-intima (a) and media-to-adventitia (b) transitions can be clearly identified.

Regardless of the algorithm used to extract arterial boundaries from B-mode frames, clinical image analysis is usually performed on a video image acquired by connecting a video grabber to the US scanner or via a DICOM interface. This implies that edge detection is performed on US frames that have already been preprocessed for visual optimization.

Generally the sequence of steps in a US scanner is as follows: the probe provides radio-frequency (RF) echo signals that are digitized with an analog-to-digital converter ( $A D C)$, and are subsequently subjected to envelope detection in order to encode echo intensity into a gray-level scale. Depending on the US device used, envelopes can pass through a series of smoothing stages in order to regularize their appearance (e.g. despeckling). A common form of non-linear signal processing, employed in commercial ultrasound scanners prior to image presentation, involves logarithmic compression of the envelopes. US systems apply compression to adapt the dynamic range of the echo signals to the smaller dynamic range of display screens, emphasizing thereby relatively weak echo levels (Dutt 1996). Compression is usually accompanied by noise level tresholding in order to regularize unwanted grey level variations in zones with low echogenicity (such as blood). Logarithmic compression alters the grey level statistics (Dutt 1995), changing the appearance and length of echo edges (Hennerici 1994), thus potentially complicating the edge identification task. Manual tracing of the arterial contours on video-grabbed images is known to be dependent on the US system characteristic in terms of thresholding, gain settings and logarithmic compression (Hoeks 1997). Moreover, a US video im- 
age contains redundant pixels due to scan conversion, i.e. interpolation occurring prior to video presentation, and possible re-sampling performed by the videograbbing device. It should be stressed that the image resolution is by no means related to the pixel distance in a video frame. Instead, factors affecting the resolution of a US image are the bandwidths of emitter, transducer and receiver, determining the shape of the RF pulse (axial resolution), in addition to the probe pitch (lateral resolution) and focusing (lateral and azimuthal resolution).

Apart from the mentioned processing steps, also saturation may introduce nonlinear effects in US imaging. When the gain settings are such that the incoming RF signal peaks exceed the dynamic range of the $A D C$, the digital RF signals will be clipped. Even if RF signals fit properly within the ADC dynamic range, brightness/contrast adjustments might yield saturated video images. If such saturation occurs for echoes coming from CCA walls (as is quite likely), edge detection and subsequently diameter assessment might be affected.

Because of the aforementioned non-linear effects, it is reasonable to imagine that the very same arterial edges would appear differently in images/signals coming from different US scanners, or even from the same scanner using different settings. This study evaluates quantitatively the effects of non-linear processing on the precision of in vivo CCA diameter measurements. With the term "non-linear processing" we will refer to logarithmic compression, noise-level thresholding and saturation. The effect of gain settings on ultrasonic diameter assessment has been recently shown (Potter 2008) for arterial tissue mimicking phantoms submerged in water, along with the effect of variations in dynamic range and probe positioning.

This study compares two basic methods for edge detection used to estimate the external (adventitia-to-adventitia) diameter of the CCA, namely the sustain-attack filter (Hoeks 1995; Meinders 2001), which utilizes exponentially decaying reference functions, and the derivative approach, using the positions of maxima in the first derivative of CCA wall echoes. Both methods are tested for precision as function of saturation and logarithmic compression of echo signals from the CCA of a group of healthy volunteers. The proposed edge detectors are implemented without any preor post-processing to regularize the diameter overlay on the US vascular frames. The exclusion of additional processing emphasizes the direct relationship between non-linear effects and ultrasonic edge detection. Both edge detectors should not be considered as complete diameter assessment algorithms, but rather as "methodological cores" to be integrated in more complex procedures involving different preand post-processing stages. The aim of this study is to test them in their stand-alone 
capability to track edges in US frames, and especially to assess the effect of saturation and compression on their outcome.

\section{3 - Materials and methods}

\subsection{1 - Acquisitions}

The common carotid arteries of 14 healthy subjects (age ranging from 15 to 40, 9 males and 5 females) were scanned in B-mode. The study was approved by the joint ethical committee of Maastricht University and Academic Hospital Maastricht. All subjects gave informed consent prior to enrolment.

A software-based radio frequency US acquisition system, called ART.LAB (ESAOTE EUROPE BV, Maastricht, The Netherlands), was used to perform B-mode scans (Brands 1999). Arteries were scanned in longitudinal sections with perpendicular incidence, and the transmit focus was set at a depth of $2 \mathrm{~cm}$, which corresponded to the whereabouts of the CCA lumen for each subject. Three subsequent acquisitions were recorded for each subject, each one covering a time interval of 6 seconds and therefore about 4-6 heart beats, depending on the heart rate of the subject. Only one acquisition had to be discarded due to an inconsistent presence of the CCA in the frames, resulting in a total of 41 recordings.

Prior to the acquisitions in supine position, each subject was given a rest period of 10 minutes. The subjects were asked to hold their breath for the brief duration of each measurement to eliminate possible variability in CCA diameter due to the respiratory cycle. Particular attention was paid to the gain settings in the ART.LAB system to avoid consistently signal saturation. This was accomplished using the saturation indicator present on the ART.LAB user interface, and also by visually checking in real-time the appearance of RF signals within a region of interest (ROI). Each acquisition resulted in storage of digitized RF echo signals. The stored file contains 180 matrixes (frame rate $30 \mathrm{fps}$, acquisition time $6 \mathrm{~s}$ ), each composed of 128 RF lines of sight, covering $40 \mathrm{~mm}$ in width and $35 \mathrm{~mm}$ in depth. The center frequency of the linear array was $7.5 \mathrm{MHz}$, while the transducer pitch was $0.315 \mathrm{~mm}$. The RF signals were sampled at a frequency of 33.3 MHz (inter-sample distance 23.1 $\mu \mathrm{m}$ ) with a dynamic range of $72 \mathrm{~dB}$ (12 bits). The 12 bits representing each (signed) value of an RF signal are stored as the most significant bits of a 16-bits word. This yields the value of 32767 as dynamic range limit. The files were subjected to off-line CCA diameter estimation using MATLAB (MathWorks, Natik, MA). The ROI within each acquired RF frame covered 64 centre lines $(20 \mathrm{~mm})$ over a depth range of 16 $\mathrm{mm}$ (i.e. 693 samples) around an initial vertical (depth) position manually set within 
the CCA lumen in the first considered frame. Over subsequent frames the ROI position was automatically updated according to the vertical displacement of the CCA as outcome of the vessel wall contour delineation process. For each RF segment in the ROI, the DC component was removed. Then, each RF matrix underwent different processing steps prior to diameter estimation, involving simulated saturation, Hilbert transformation to compute the envelope, logarithmic compression and noise level tresholding.

\subsection{2 - Envelopes}

The envelope of each RF signal within the ROI was computed by means of time domain Hilbert transformation. To generate complex analytic signals the RF signals were convolved with an impulse response having 7 non-zero samples on both the positive and negative side in order to minimize amplitude and phase errors (Brands 1997). The instantaneous envelope of the RF signal is the absolute value of the analytic signal. Fig. 3.2a presents a segment of an RF signal with a superimposed envelope.

\subsection{3 - Saturation simulation}

All US frames were acquired without saturation. Each ROI is pre-amplified to scale its absolute maximum to the dynamic range limit. Subsequently, the ROI is subjected to a second scaling process to achieve a prescribed percentage of saturation for either RF or envelope signals. Fig. 3.2d and 3.2g show an RF signal and its envelope at $50 \%$ saturation, respectively.

\subsection{4 - Logarithmic compression and noise level thresholding}

US systems normally apply logarithmic compression on echo envelopes in order to improve visualization. This decreases the relative amplitude of bright strong echoes with respect to weakly backscattering structures, allowing the latter ones to be properly visualized on screen. 

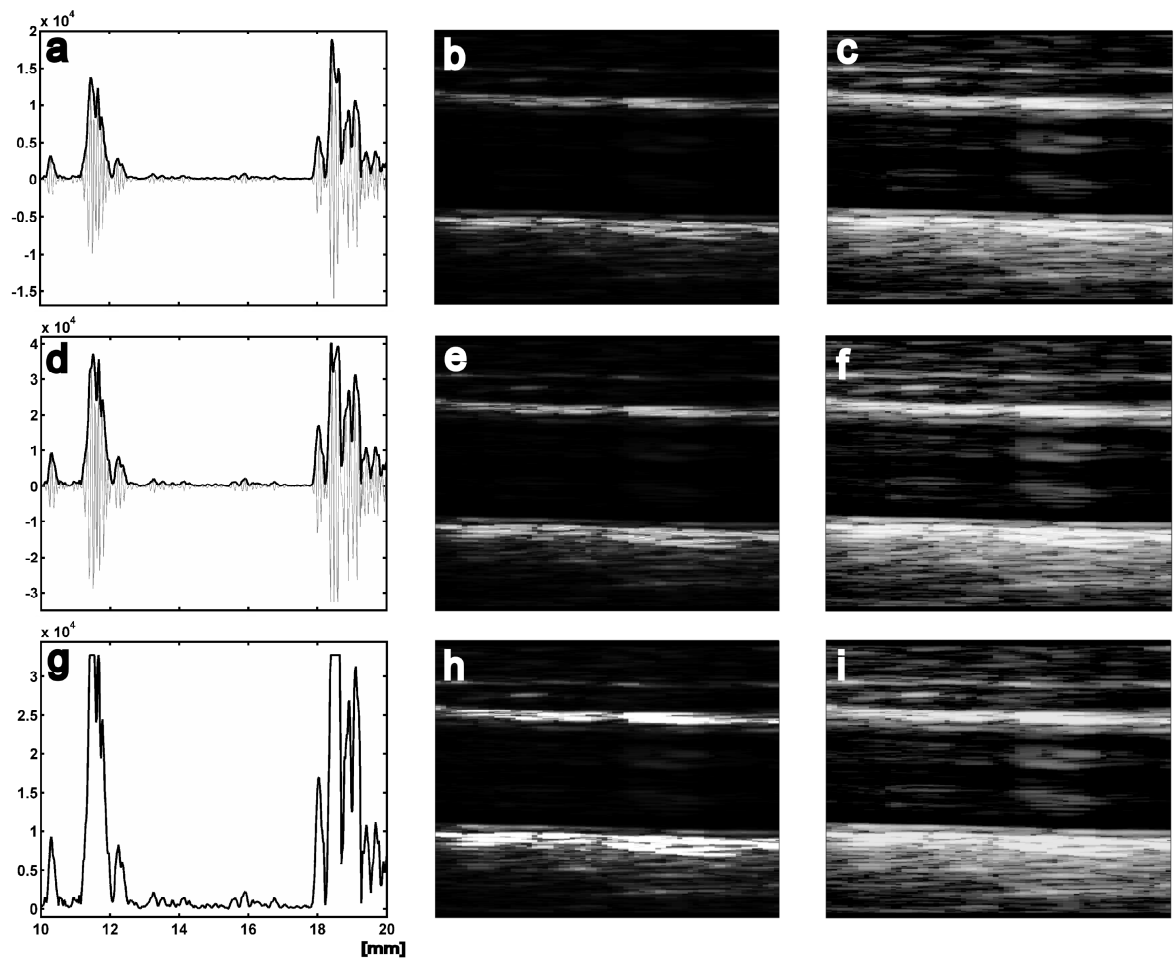

Fig. 3.2 - Effects of saturation and logarithmic compression. Top row (a, b, c): unsaturated signals and ROI, consisting of 64 lines of sight $(20 \mathrm{~mm})$ centered on the carotid lumen and covering a depth of $16 \mathrm{~mm}$. Center (d, e, f) and bottom (g, h, i) row: 50\% RF and 50\% envelope saturation, respectively, for signals and ROI. All frames are normalized to the maximum signal level within the ROI. The center column shows linear images, the right column log-compressed ones.

Also noise level thresholding is performed to eliminate spurious grey-level variations in zones having low echogenicity, such as blood. In this study, logarithmic compression and thresholding were achieved with:

$$
\left\{\begin{array}{c}
E_{\mathrm{LC}}=T h+20 \log _{10}\left(\frac{E}{E_{\mathrm{Max}}}+10^{-5}\right) \\
\text { if } \mathrm{E}_{\mathrm{LC}}<0, \mathrm{E}_{\mathrm{LC}}=0
\end{array}\right.
$$

where $E_{L C}$ is the logarithmically compressed and thresholded envelope, Th the noise level threshold expressed in $\mathrm{dB}, E$ the unprocessed envelope signal and $E_{\text {Max }}$ is the 
maximum value of the unprocessed envelopes in the ROI. The $10^{-5}$ term is added to ensure a non-zero argument of the logarithm. Thresholding is performed after logarithmic compression by forcing all negative values of $E_{L C}$ to zero. In this study the value of Th was set to $40 \mathrm{~dB}$. For notational convenience, the original envelopes (and images) will be referred to as "linear", whereas the logarithmically compressed (and noise-level thresholded) ones will be called "log-compressed". Fig. 3.2 shows linear $(b, e, h)$ and log-compressed $(c, f, i)$ images in various saturation conditions.

\subsection{5 - Automatic edge detector \#1: sustain-attack filter}

The CCA diameter was independently assessed for every echo line within the ROI. In each signal the near and far wall positions (media-adventitia interfaces) were localized using the so-called "sustain-attack" low pass filter (Hoeks 1995; Meinders 2001), providing signal thresholds related to the amplitude of the nearby arterial wall echoes. The sustain-attack filter is based on the generation of a reference signal, SA(d), which decays exponentially as function of depth $d$ (expressed in sample points). Whenever the $S A(d)$ falls below the local envelope, it is reset to the instantaneous amplitude of the echo signal (Fig. 3.3a).

$$
\left\{\begin{aligned}
\text { if } S A(d)>E(d), S A(d)=\left(1-\frac{c}{2 \cdot f_{S} \cdot d_{E X P}}\right) \cdot S A(d-1) \\
\text { if } S A(d) \leq E(d), S A(d)=E(d)
\end{aligned}\right.
$$

In equation (3.2), $c$ is the speed of sound in tissue $(1540 \mathrm{~m} / \mathrm{s})$ and $f_{S}$ the sampling frequency in $\mathrm{Hz} . d_{E X P}$ is the decay parameter, related to the depth-dependent variations in echo amplitude. For peripheral applications, a value of $7 * 10^{-3} \mathrm{~m}$ is appropriate for the decay parameter (Hoeks 1995). The reference signal is always related to the last dominant echo while it follows gradual changes in echo level along the line of sight. From the reference signal, a dynamic threshold level DTR $\mathrm{L}_{\text {LIN }}$ is generated as function of depth for linear envelopes:

$$
\operatorname{DTR}_{\mathrm{LIN}}(\mathrm{d})=\alpha \cdot \mathrm{SA}(\mathrm{d})
$$


where $\alpha$ is a constant between 0 and 1. For this study, $\alpha$ is fixed at 0.5 (Hoeks 1995). The anterior adventitial wall position is assigned to the first point from the

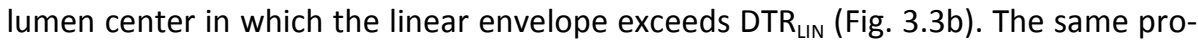
cedure is followed for the posterior wall but with the reference signal and DTR generated in a backward direction. The dynamic threshold level adapts to the nearby signal level, working similarly for both bright and weak echoes. This is because $S A(d)$ is inherently tied to the peak amplitudes nearby the lumen. In this way, the SAF edge detection outcome is not sensitive to variations in gain settings by different sonographers.

For a log-compressed envelope $E_{L C}$ (Fig. 3.4a and 3.4b), the logarithmically compressed dynamic threshold level $\mathrm{DTR}_{\mathrm{LOG}}$ is derived from $\mathrm{SA}(\mathrm{d})$ as:

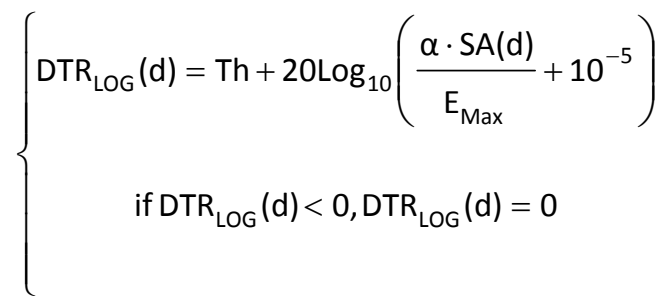

where $E_{\text {Max }}$ is the maximum value of the unprocessed envelopes in the ROI.

DTR $_{\text {LOG }}$ is also generated in both forward and backward directions (Fig. 3.4b) for each line within the ROI (for near and far walls). The adventitial wall positions are assigned to the first points from the lumen center in which the log-compressed envelope is higher than $\mathrm{DTR}_{\mathrm{LOG}}$.

\subsection{6 - Automatic edge detector \#2: derivative maximum}

Both for the linear and log-compressed case, the outcome of the sustain-attack filter was compared to the results obtained with derivative analysis of the envelope signals. Starting from the absolute peak of the envelope at each arterial wall (i.e. the points at which SA(d) starts its exponential decay towards the lumen center), segments of the envelope signal reaching $1.5 \mathrm{~mm}$ towards the lumen were analyzed (Fig. 3.3c and 3.4c). For each segment, the point with the maximum derivative (derivative direction from lumen to wall) was associated with the adventitial wall position (Fig. 3.3d and 3.4d). 

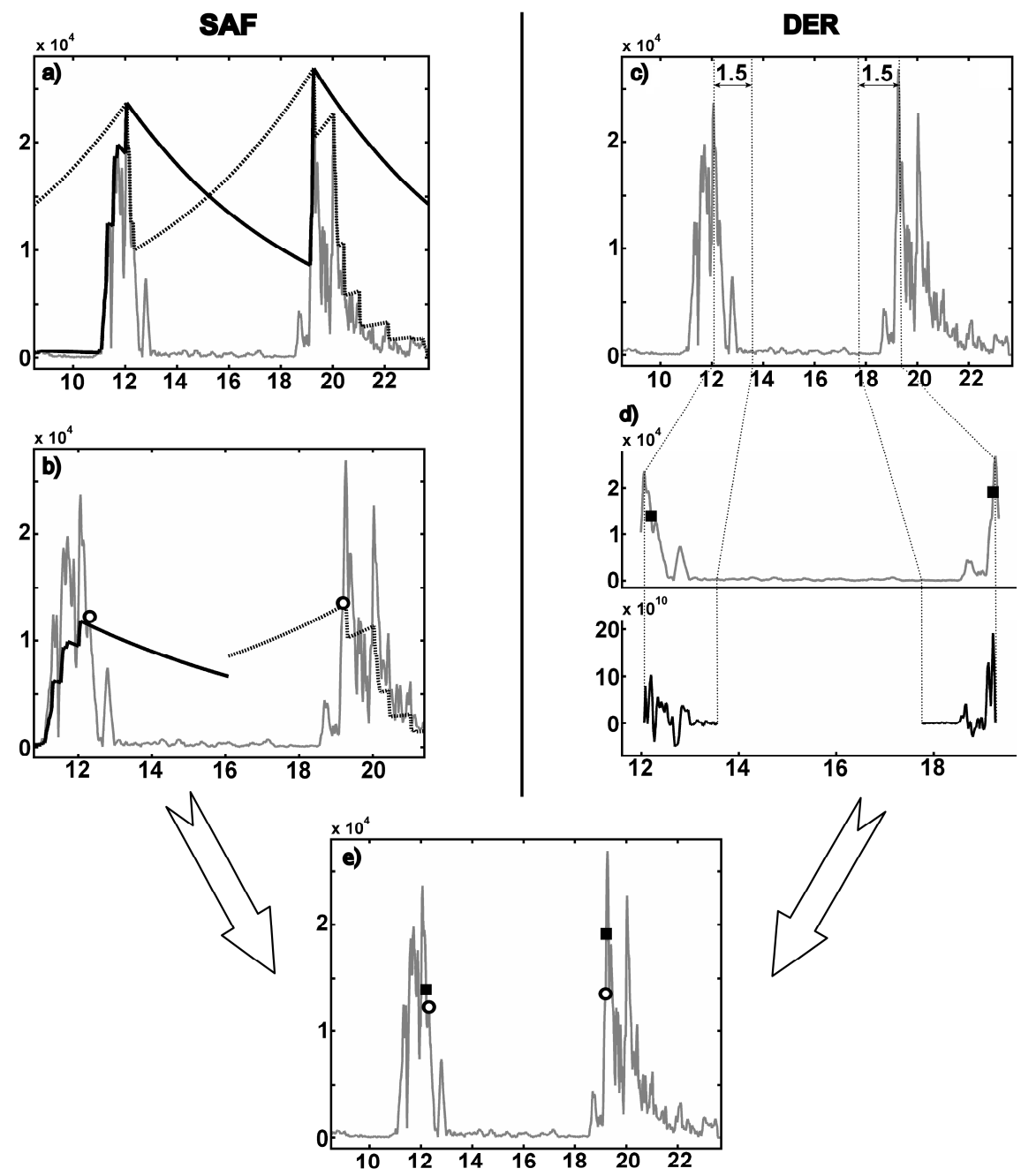

Fig. 3.3 - Sustain-attack filter (SAF) versus derivative approach (DER) for diameter estimation on linear US envelopes. a) Near wall (solid line) and far-wall (dotted line) SAF reference functions SA(d). b) Near wall (solid line) and far wall (dotted line) DTR LIN tracks used to estimate adventitial wall positions (white circles). c) The envelope segments corresponding to the first $1.5 \mathrm{~mm}$ towards the lumen from the adventitial peaks are processed for DER. d) Top panel: enlarged segment of CCA lumen; bottom panel: directional (from lumen center outwards) derivative tracks; the black squares in the top panel of figure d) correspond to the positions of the derivative peaks. e) Comparison between adventitial wall positions as estimated with SAF (white circles) and DER (black squares) for a linear envelope. 

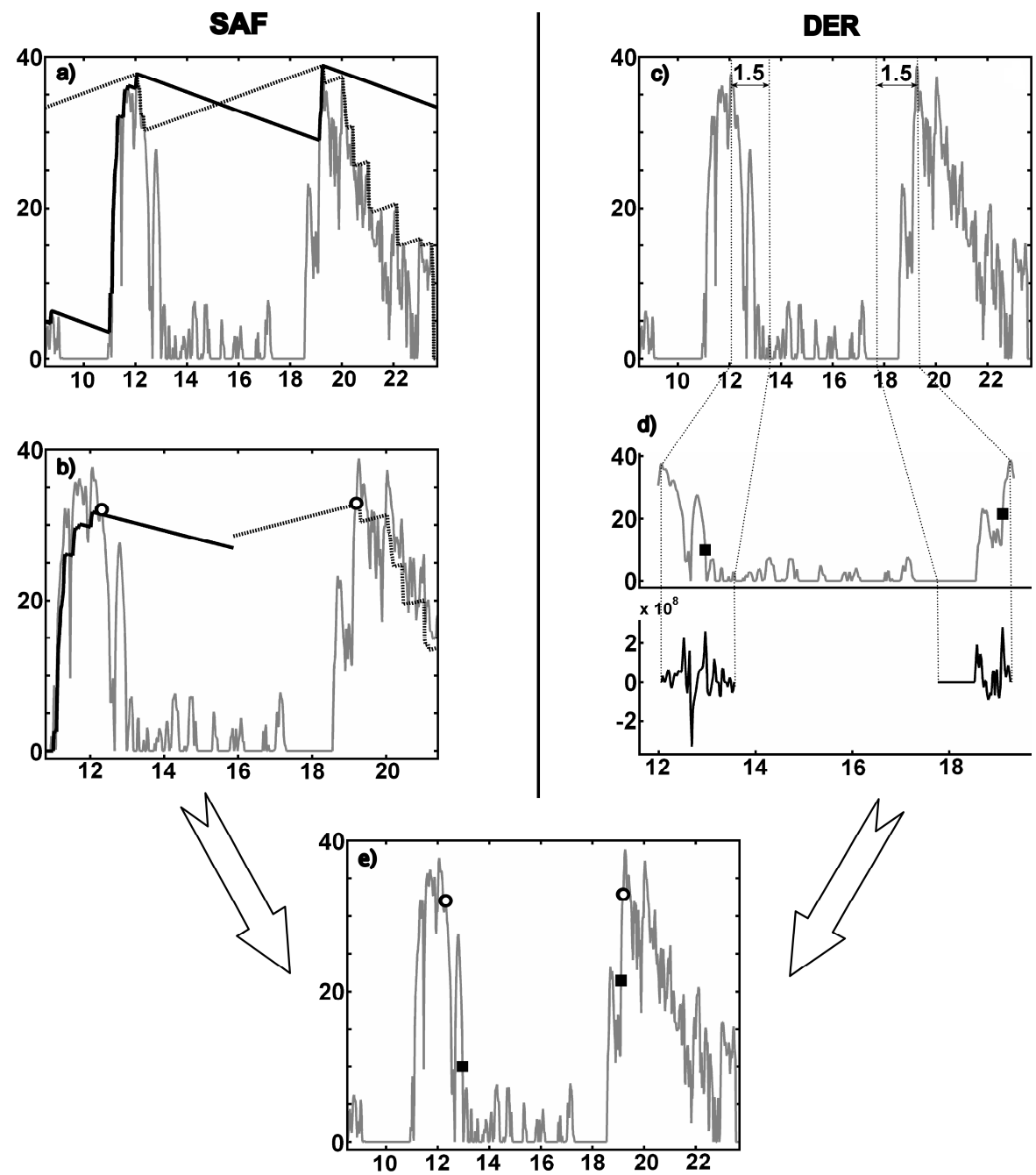

Fig. 3.4 - Sustain-attack filter (SAF) versus derivative approach (DER) to estimate wall positions for log-compressed US envelopes. The analysis is similar as described in Fig. 3.3 for linear signals, but in this case the threshold functions are computed taking into account the logcompression characteristic. Panel a) shows the log-compressed version of SA(d) for each wall,

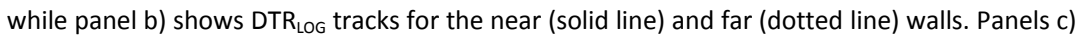
and d) illustrate the derivative approach. Panel e) compares adventitial wall positions as estimated with SAF (white circles) and DER (black squares) for a log-compressed envelope.

\subsection{7 - CCA diameter measurements}

The instantaneous adventitia-to-adventitia diameter follows directly from the distance between the identified adventitia-media transitions. For both diameter estimators, the analysis is carried out without any form of post-processing (e.g. smooth- 
ing) on the CCA wall positions obtained at each line of sight within the ROI (Fig. 3.5). In this way the differences in diameter estimates and precision can directly be related to the applied non-linear processing simulations.
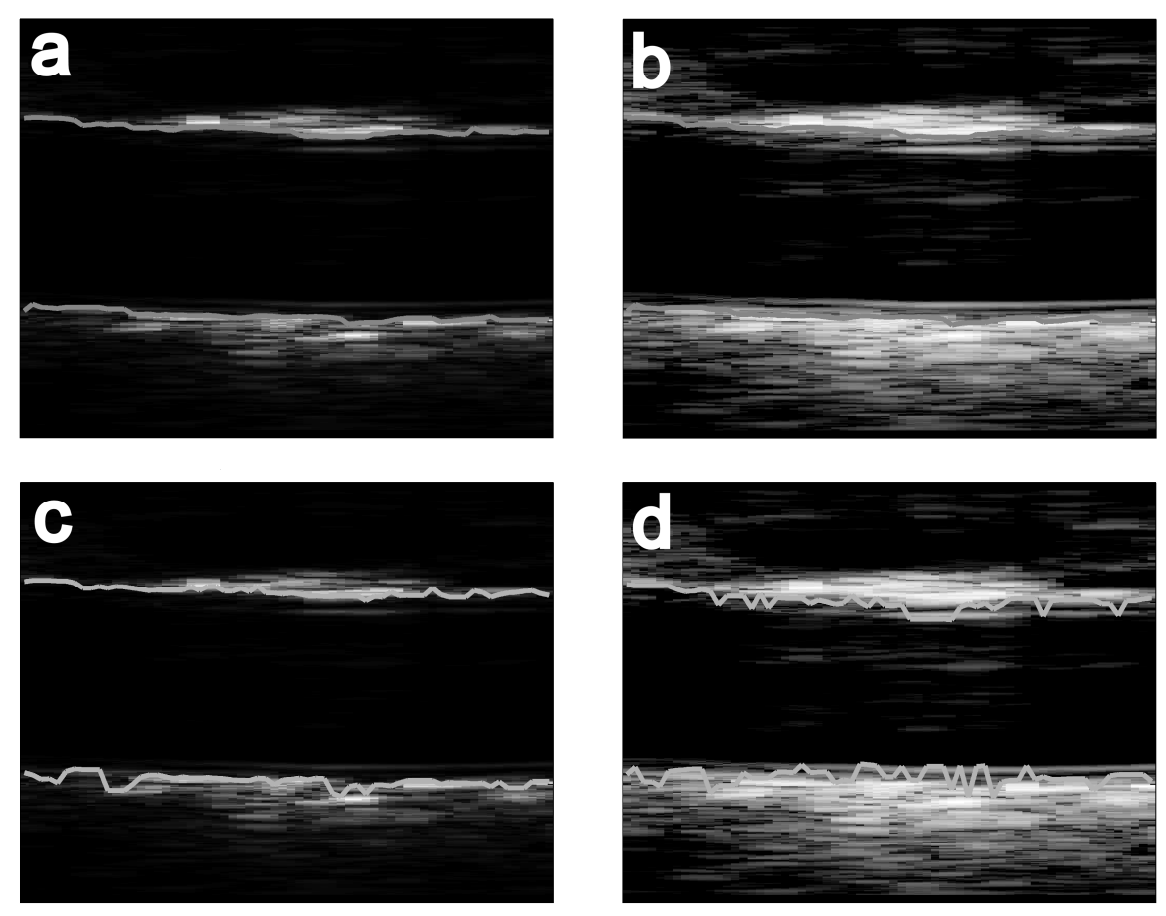

Fig. 3.5 (color version on page 149) - a) and b) Sustain-attack filter applied to a linear and a log-compressed image, respectively. c) and d) Derivative approach applied to a linear and a log-compressed image, respectively. All images are unsaturated, and refer to a ROI consisting of 64 lines of sight, centered on the carotid lumen and covering a depth of $16 \mathrm{~mm}$. No postprocessing was performed on the results, in order to properly assess the effect of different pre-processing conditions on the outcome of the two basic edge detectors.

Table 3.1 presents the different saturation cases considered, each one listing the processing steps in order of occurrence, starting from the RF signal. NS refers to the case with no saturation. S25RF and S50RF refer to RF signal saturation, simulating an overexposure of the signal prior to analog-to-digital conversion. S25E and S50E refer to envelope saturation, simulating overexposure of the displayed image (gain and brightness controls).

Diameter estimation was performed with both the described basic edge detectors. Each analysis was carried out for both linear and log-compressed envelopes. For the sustain-attack filter, the latter also implies compression of the threshold function 
DTR (equation 3.4). Each processing case results in a diameter waveform for both edge detectors (Fig. 3.6).

Table 3.1 - Saturation simulations

\begin{tabular}{ll}
\hline \hline Processing case & Processing steps \\
\hline NS & envelope computation, CCA diameter estimation \\
S25RF & saturation $25 \%$, envelope computation, diameter estimation \\
S25E & envelope computation, saturation $25 \%$, diameter estimation \\
S50RF & saturation $50 \%$, envelope computation, diameter estimation \\
S50E & envelope computation, saturation $50 \%$, diameter estimation \\
\hline \hline
\end{tabular}

Each saturation case acronym is associated with a specific sequence of processing steps.
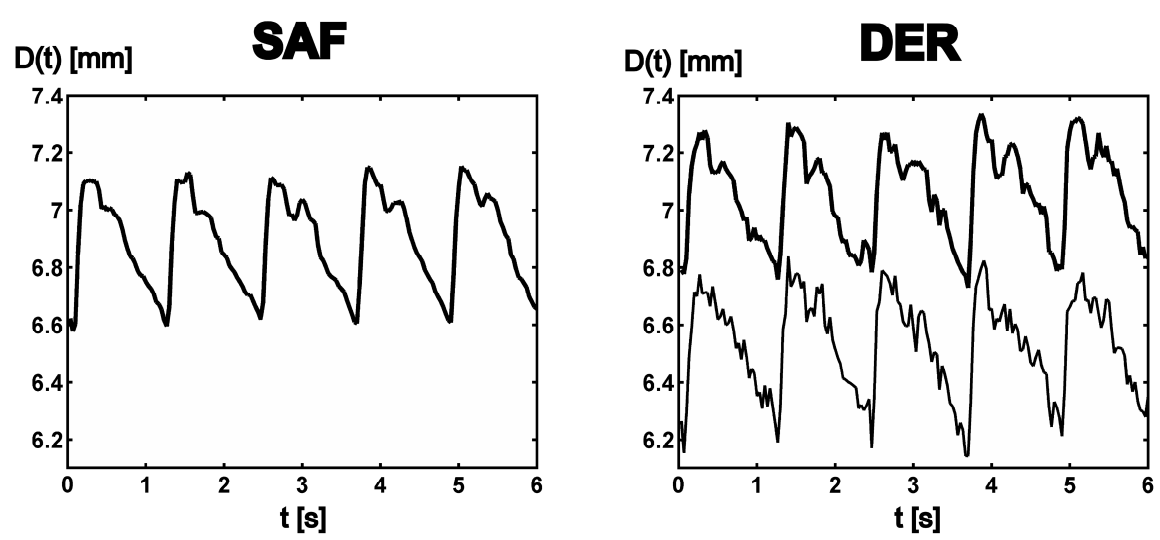

Fig. 3.6 - Diameter waveforms $(30 \mathrm{~Hz})$ obtained from unsaturated signals for the sustainattack filter (left panel, SAF) and derivative approach (right panel, DER) for the same US recording. Even though no pre- or post-processing is used, the sustain-attack filter provides smoother and more realistic waveforms than the derivative approach. The linear (thick line) and logarithmically compressed (thin line) cases totally overlap for the sustain-attack filter, while the derivative approach is clearly affected by logarithmic compression, inducing a 600 $\mu \mathrm{m}$ shift of the diameter waveform as well as variations in its profile.

Each sample of the waveform is calculated as the average of the 64 diameter values within the ROI at a specific time instant. The diameter sampling frequency was thus $30 \mathrm{~Hz}$, the same as the B-mode frame rate. The detection of diastolic and systolic diameter points was performed semi-automatically, and the values were stored in a separate database. The total number of recordings $\mathrm{Nr}$ was 41 . Each one of them contained a variable number of beats $\mathrm{Br}$. The total number of recorded beats $\mathrm{Nb}$ equaled 222. Intra-recording average values were calculated for diastolic $\left(\bar{D}_{D}\right)$, 
systolic $\left(\bar{D}_{s}\right)$ and pulse $\left(\bar{D}_{p}\right)$ diameter. The intra-recording variability, defined as the root mean square (RMS) deviation from the recording average, was calculated as:

$$
\sigma_{R, X}=\sqrt{\frac{1}{N r} \sum_{k=1}^{N r} \frac{1}{B r(k)-1} \sum_{j=1}^{B r(k)}\left[D_{x}(j, k)-\overline{D x}_{x}(k)\right]^{2}}
$$

where $X$ can stand for diastolic (D), systolic (S) or pulse (P) diameter.

Intra-subject values for diameter and root-mean-square error were also calculated as averages over $N s=14$ subjects. The number of recordings per subject was $\mathrm{R}=3$ except for one, where $\mathrm{R}=\mathbf{2}$. Intra-subject means for diastolic, systolic and pulse diameter were obtained by averaging the intra-recording means over the recordings pertaining to a specific subject. The intra-subject variability was then computed as:

$$
\sigma_{B, X}=\sqrt{\frac{1}{N s} \sum_{i=1}^{N s} \frac{1}{R(i)-1} \sum_{k=1}^{R(i)}\left[\bar{D}_{x}(k)-\frac{1}{R(i)} \sum_{a=1}^{R(i)} \bar{D}_{x}(a)\right]^{2}}
$$

where $X$ stands for $D, S$ or $P$.

\section{4-Results}

Fig. 3.7 shows the results for the intra-recording variability for diastolic $\left(\sigma_{R, D}\right)$, systolic $\left(\sigma_{R, S}\right)$ and pulse $\left(\sigma_{R, P}\right)$ diameter, plotted as function of saturation conditions and log-compression. The sustain-attack filter has a better precision for all cases, with an intrarecording variability that is on average about $40 \mu \mathrm{m}$ less than the one obtained using the derivative approach. In all processing cases the variability of diastolic values is higher than that of systolic diameters. Logarithmic compression and noise level thresholding do not affect intra-recording variability for the sustain-attack filter. On the other hand, intra-recording variations in diastolic and pulse diameter obtained with the derivative approach increase markedly for the log-compressed case compared to the linear situation. Increasing the saturation level diminishes the difference in intra-recording variability between sustain-attack filter and derivative analysis, even though the former is consistently less variable than the latter. 

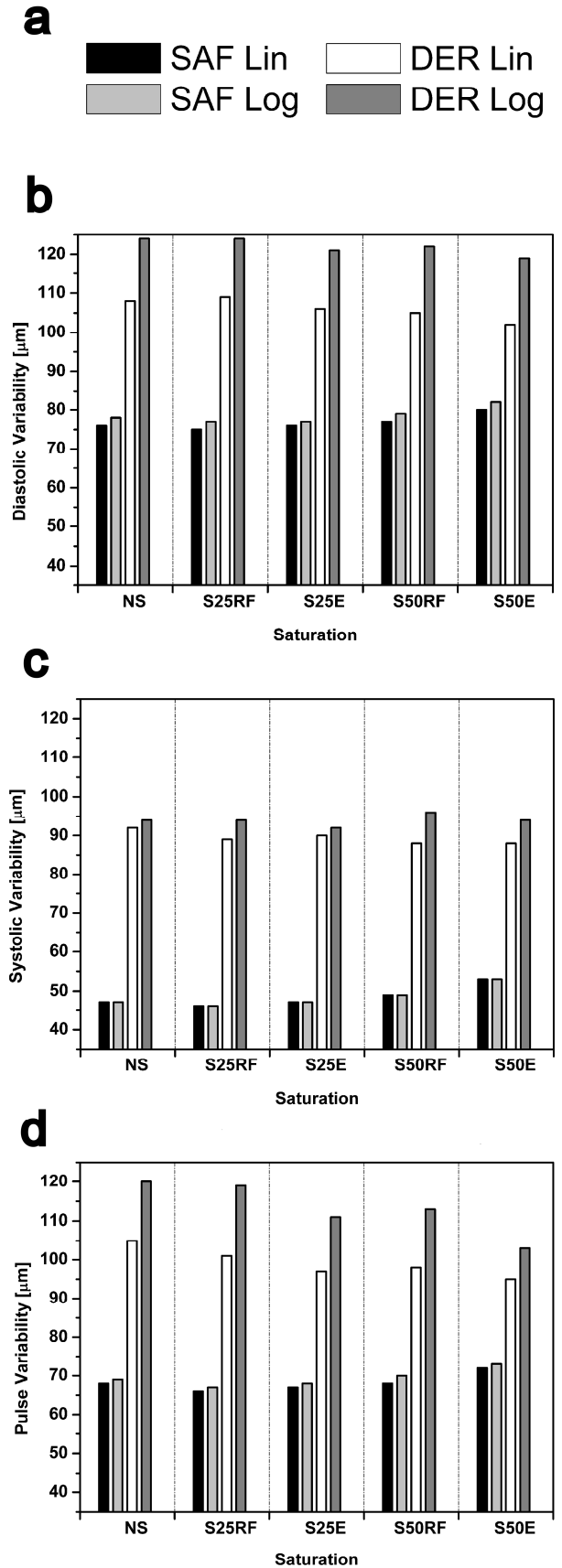

Fig. 3.7 - Intra-recording variability of estimated CCA diameter based on 41 recordings, covering 222 beats. a) Color legend. b) Diastolic intra-recording variability $\sigma_{R, D}$. c) Systolic intrarecording variability $\sigma_{R, S}$. d) Pulse intra-recording variability $\sigma_{R, P}$. 
a

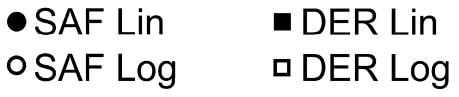

b
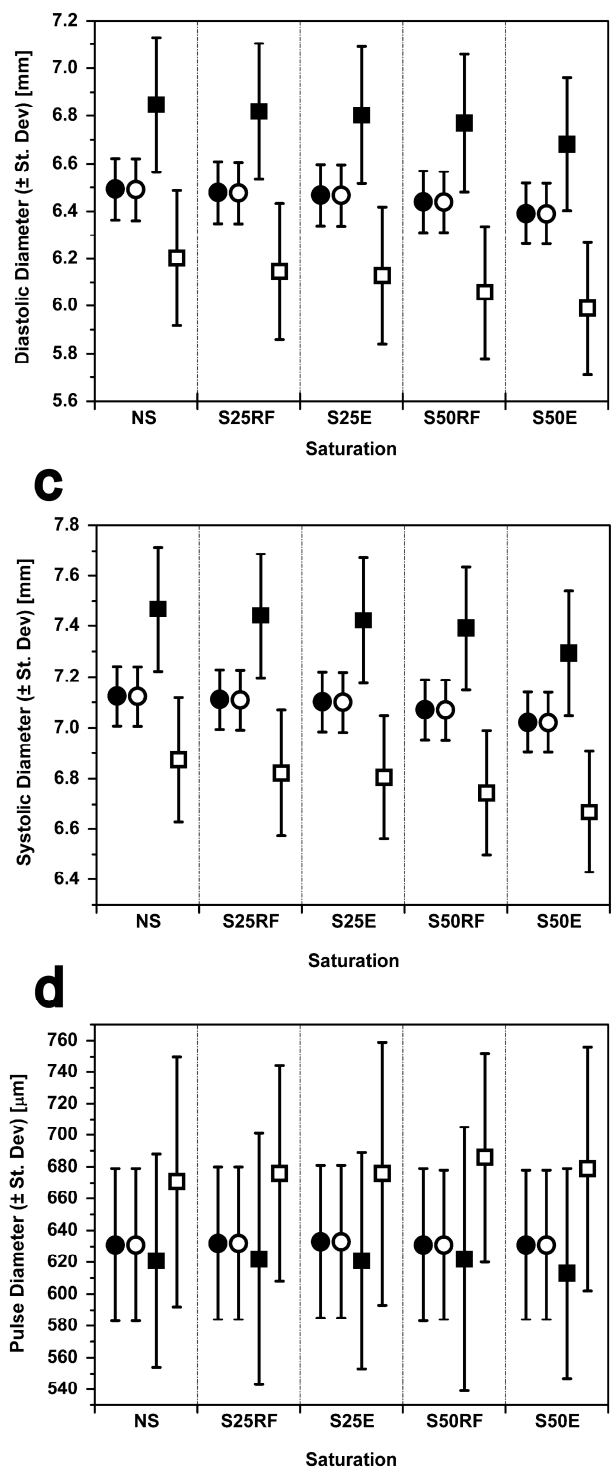

Fig. 3.8 - Intra-subject analysis of CCA diameter for 14 subjects. a) Symbol legend. b) Diastolic diameter. c) Systolic diameter. d) Pulse diameter. Whiskers represent the intra-subject variabilites $\sigma_{\mathrm{B}, \mathrm{D}}, \sigma_{\mathrm{B}, \mathrm{S}}$ and $\sigma_{\mathrm{B}, \mathrm{P}}$. 
Fig. 3.8 shows the intra-subject mean diameter values for diastolic, systolic and pulse diameter, plotted as function of saturation conditions and log-compression. The whiskers represent the intra-subject standard deviation obtained for the diastolic $\left(\sigma_{B, D}\right)$, systolic $\left(\sigma_{B, S}\right)$ and pulse $\left(\sigma_{B, P}\right)$ values. Logarithmic compression does not affect diameter values obtained with the sustain-attack filter. The derivative approach is rather sensitive to it: in the linear cases diastolic and systolic diameters are higher than with the sustain-attack filter, while in the log-compressed cases they are lower. This can also be observed in Figs. 3.3e, 3.4e, 3.5 and 3.6. Using the derivative edge detector, the average difference in estimated diameter between linear and log-compressed images is about $600 \mu \mathrm{m}$ for both diastolic and systolic phase. This means for the derivative approach a variation of about $10 \%$ in estimated external diameter values, depending on the pre-processing conditions of the image. The pulse diameter is higher in the log-compressed case and smaller in the linear case when computed using the derivative approach, again with a $10 \%$ difference. These drawbacks are not present when using the sustain-attack filter, that yields consistent values for diastolic, systolic and pulse diameter for both linear and-log compressed images.

Diastolic and systolic diameter values obtained with the sustain-attack filter decrease on average $100 \mu \mathrm{m}$ when passing from the unsaturated case to the simulated maximum saturation. For the derivative approach this decrease is about 200 $\mu \mathrm{m}$. Since the observed decreases for systolic and diastolic diameter are similar, the pulse diameter is hardly affected by saturation.

The derivative approach exhibits a higher intra-subject variability than the sustainattack filter, implying a better repeatability for the latter. Saturation and logcompression have no relevant effect on intra-subject variability for both edge detectors.

\section{5 - Discussion}

Automatic delineation of the common carotid artery walls based on edge detection in B-mode ultrasound is sensitive for saturation and logarithmic compression. The sustain-attack filter, compared to the derivative approach, provides results that are more robust, consistent and less affected by non-linearities.

Logarithmic compression and saturation can have an important effect on noninvasive CCA diameter assessment when using approaches based on the grey level 
gradient. CCA diameter overlays on images (Fig. 3.5) and diameter waveforms (Fig. 3.6) acquired with the sustain-attack filter are much smoother and realistic than with the derivative approach. It should be noted that the comparison between both methods is made for diameter waveforms without any smoothing to improve consistency. Intra-recording and intra-subject variations, together with the assessed diameter values, show that the sustain-attack filter is more stable and precise than the derivative approach and yields more reproducible estimates. Logarithmic compression does not affect the variability of the sustain-attack filter, while it does for the derivative approach. Moreover, logarithmic compression also induces an average deviation of about $600 \mu \mathrm{m}$ (i.e. 10\%) in the estimated diameter values when using derivative analysis. This does not happen for the sustain-attack filter due to its inherent possibility to integrate the compression characteristic of the system in the formulation of the reference functions. The fact that for the derivative approach log-compression decreases the assessed diameter is explainable because compression shifts the maximum-slope points towards the lumen (Figs. 3.3 and 3.4). In the present study, the utilized logarithmic compression and noise-level tresholding function (equation 3.1) was exactly known. In a clinical environment the logcompression characteristics of the US system can be derived a posteriori from the acquired images (Dutt 1996).

It is worth noting that the sustain-attack filter only requires placing of a seed point in the lumen. The derivative approach required the selection of two $1.5 \mathrm{~mm}$ ranges near the wall-lumen interfaces to avoid possible intra-luminal reverberations affecting its outcome.

The position of the media-adventitia interface is of importance not only for the estimation of diameter, but also for non-invasive assessment of intima-media thickness (Van Bortel 2001; Touboul 2007). Therefore, a consistent estimation of external diameter independent of log-compression and saturation conditions, as provided by the sustain-attack filter, is also of high interest for algorithms aiming at intima-media thickness estimation. From this point of view, also small diameter deviations (100 to $200 \mu \mathrm{m}$ ) introduced by saturation are relevant, because such small variations in the estimated adventitia-to-adventitia distance, though not relevant for diameter estimation, can affect the assessment of intima-media thickness.

Our study focuses on the discrepancies between the two edge detection methods in vivo, and on the effect that non-linear processing has on their precision. No conclusions are drawn about the accuracy of both methods in vivo, since the real CCA diameter value cannot be estimated. In order to have some insight on the accuracy of the two algorithms, we performed some additional measurements on a phantom composed of two nylon wires (diameter $80 \mu \mathrm{m}$ each), spaced at $5 \mathrm{~mm}$ and sub- 
merged in distilled water (speed of sound $1490 \mathrm{~m} / \mathrm{s}$ ). The "lumen" between the two wires was centered at $2 \mathrm{~cm}$ from the probe surface, and so was the transmit focus. The resulting distance between the trailing edge of the upper echo and the leading edge of the lower echo in the US phantom image is $4.6 \mathrm{~mm}$, accounting for the thicknesses of the two nylon wires and for the resolution of the US system (in the order of $330 \mu \mathrm{m}$ at $50 \%$ of the peak amplitude). For linear signals, the SAF and derivative approach present similar accuracy, SAF being slightly better $(4.46 \mathrm{~mm}$ for SAF and $4.43 \mathrm{~mm}$ for DER). The bias (about $150 \mu \mathrm{m}$ ) in the linear case is well below the US axial resolution. Saturation did not introduce relevant variations in the assessed diameter values (the maximum saturation-induced variation was in the order of $30 \mu \mathrm{m}$ for both methods). Logarithmic compression did not affect the SAF outcome at all, while it induced a significant decrease in the outcome of DER $(4.3 \mathrm{~mm}$ on average). Thus, the sustain-attack filter is insensitive to log compression not only in terms of precision, but also in terms of accuracy, while the derivative approach is affected by such non-linear effect. However, accuracy measures in a phantom setup give only a vague idea of how the accuracy will be in vivo: nylon wires in water produce strong specular reflections with a very small amount of speckle noise, whereas living tissues reflect sound in a much more distributed and irregular way, making it difficult to quantify the extent of trailing edges. Due to the impracticability of accuracy estimates in vivo, the precision is clinically the most important index, representing the reliability and repeatability of the measurements.

In conclusion, this study demonstrates that the sustain-attack filter as edge detector provides non invasive CCA US image segmentation which is insensitive to logarithmic compression and less influenced by saturation. It also emphasizes the importance of knowing the logarithmic compression characteristics of the ultrasound system and of avoiding overexposure of signals and images during US acquisitions. Since many of the current methods for automatic segmentation of US frames still rely on processing of video images, the results of this study are of relevance for the clinical validity and reliability of US-based CCA image analysis. The authors believe that methods utilizing RF echo signals are more suited in order to univocally correlate the measured features to the underlying vascular structure, since they can bypass the signal processing necessary for visual optimization.

\section{References}

Brands PJ, Hoeks APG, Ledoux LAF, Reneman RS. A radio frequency domain complex cross-correlation model to estimate blood flow velocity and tissue motion by means of ultrasound. Ultrasound in Medicine \& Biology 1997; 23: 911-920. 
Brands PJ, Hoeks APG, Willigers J, Willekes C, Reneman RS. An integrated system for the non-invasive assessment of vessel wall and hemodynamic properties of large arteries by means of ultrasound. European Journal of Ultrasound 1999; 9: 257-266.

Cheng D, Schmidt-Trucksass A, Cheng K, Burkhardt H. Using snakes to detect the intimal and adventitial layers of the common carotid artery wall in sonographic images. Computer Methods and Programs in Biomedicine 2002; 67: 27-37.

Craiem D, Chironi G, Gariepy G, Miranda-Lacet J, Levenson J, Simon A. New monitoring software for larger application of brachial artery flow-mediated vasodilatation measurements. Journal of Hypertension 2007; 25: 133-140.

De Groot E, Hovingh K, Wiegman A, Duriez P, Smit AJ, Fruchart JC, Kastelein JP. Measurement of arterial wall thickness as a surrogate marker for atherosclerosis. Circulation 2004; 109: 33-38.

Delsanto S, Molinari F, Giusetto P, Liboni W, Badalamenti S, Suri JS. Characterization of a completely user-independent algorithm for carotid artery segmentation in 2-D ultrasound images. IEEE Transactions On Instrumentation And Measurement 2007; 56: 1265-1274.

Dutt V, Statistical analysis of ultrasound echo envelope. Mayo Graduate School, Rochester, (1995). PhD Thesis.

Dutt V, Greenleaf JF. Adaptive speckle reduction filter for log-compressed B-Scan images. IEEE Transactions On Medical Imaging 1996; 15: 802-813.

Graf S, Gariepy J, Massoneau M, Armentano RJ, Mansour S, Barra JG, Simon A, Levenson J. Experimental and clinical validation of arterial diameter waveform and intimal media thickness obtained from B-mode ultrasound image processing. Ultrasound in Medicine \& Biology 1999; 25: 1353-1363.

Hennerici MG, Steinke W. Accuracy of high-resolution ultrasound imaging for quantitative assessment of early carotid atherosclerosis. Cerebrovascular Diseases 1994; 4: 109-113.

Hoeks APG, Di X, Brands PJ, Reneman RS. An effective algorithm for measuring diastolic artery diameter. Archives Of Acoustics 1995; 20: 65-76.

Hoeks APG, Willekes C, Boutouyrie P, Brands PJ, Willigers JM, Reneman RS. Automated detection of local artery wall thickness based on $\mathrm{m}$-line signal processing. Ultrasound in Medicine \& Biology 1997; 23: 10171023.

Loizou CP, Pattichis CS, Pantziaris M, Tyllis T, Nicolaides A. Quality evaluation of ultrasound imaging in the carotid artery based on normalization and speckle reduction filtering. Medical and Biological Engineering and Computing 2006; 44: 414-426.

Mattace-Raso FUS, Van Der Cammen TJM, Hoffman A, Van Popele NM, Bos ML, Schalekamp MADH, Asmar R, Reneman RS, Hoeks APG, Breteler MMB, Witteman JCM. Arterial stiffness and risk of coronary heart disease and stroke - The Rotterdam study. Circulation 2006; 113: 656-663.

Meinders JM, Brands PJ, Willigers JM, Kornet L, Hoeks APG. Assessment of the spatial homogeneity of artery dimension parameters with high frame rate 2-D B-mode. Ultrasound in Medicine \& Biology 2001; 27: 785-794.

Meinders JM, Hoeks APG. Simultaneous assessment of diameter and pressure waveforms in the carotid artery. Ultrasound in Medicine \& Biology 2004; 30: 147-154.

Potter K, Reed CJ, Green DJ, Hankey GJ, Arnolda LF. Ultrasound settings significantly alter lumen and wall thickness measurements. Cardiovascular Ultrasound 2008; 6: DOI: 10.1186/1476-7120-6-6. 
Reneman RS, Meinders JM, Hoeks APG. Non-invasive ultrasound in arterial wall dynamics in humans: what we have learned and what remains to be solved. European Heart Journal 2005; 26: 960-966.

Samijo SK, Willingers JM, Barkhuysen R, Kitslaar PJEHM, Reneman RS, Brands PJ, Hoeks APG. Wall shear stress in the human common carotid artery as function of age and gender. Cardiovascular Research 1998; 39: 515-522.

Selzer RH, Hodis HN, Kwong-Fu H, Mack WJ, Lee PL, Liu CR, Liu CH. Evaluation of computerized edge tracking for quantifying intima-media thickness of the common carotid artery from B-mode ultrasound images. Atherosclerosis 1994; 111: 1-11.

Sonka M, Liang W, Lauer RM. Automated analysis of brachial ultrasound image sequences: early detection of cardiovascular disease via surrogates of endothelial function. IEEE Transactions On Medical Imaging 2002; 21: 1271-1279.

Stadler RW, Taylor JA, Lees RS. Comparison of B-mode, M-mode and echo-tracking methods for measurement of the arterial distension waveform. Ultrasound in Medicine \& Biology 1997; 23: 879-887.

Stein JH, Korkarz CE, Mays ME, Douglas PS, Palta M, Zhang H, LeCaire T, Paine D, Gustafson D, Fan L. A semiautomated ultrasound border detection program that facilitates clinical measurement of ultrasound carotid intima-media thickness. Journal of the American Society of Echocardiography 2005; 18: 244-251.

Touboul P-J, Hennerici MG, Meairs S, Adams H, Amarenco P, Bornstein N, Csiba L, Desvarieux M, Ebrahim S, Fatar M, Hernandez Hernandez R, Jaff M, Kowantor S, Prati P, Rundek T, Sitzer M, Schminke U, Cardif JC, Taylor A, Vicaut E, Woo KS, Zannad F, Zureik M. Mannheim carotid intima-media thickness consensus (2004-2006). Cerebrovascular Diseases 2007; 23: 75-80.

Van Bortel L, Vanmolkot FH, Van der Heijden-Spek JJ, Bergu M, Staessen JA, Hoeks APG. Does B-mode common carotid artery intima-media thickness differ from m-mode? Ultrasound in Medicine \& Biology 2001; 27: 1333-1336. 


\section{CHAPTER 4}

\section{A new algorithm for the automatic recognition of the common carotid artery in longitudinal ultrasound scans}

The content of this chapter is based on the publication: Rossi AC, Brands PJ, Hoeks APG. Automatic recognition of the common carotid artery in longitudinal ultrasound B-mode scans. Medical Image Analysis 2008; 12: 653-665. 


\section{1-Abstract}

Many morphological and dynamic properties of the common carotid artery (CCA), e.g. lumen diameter, distension and wall thickness, can be measured non-invasively with ultrasound (US) techniques. As common to other medical image segmentation processes, this requires as a preliminary step the manual recognition of the artery of interest within the ultrasound image. In real-time US imaging, such manual initialization procedure interferes with the difficult task of the sonographer to select and maintain a proper image scan plane. Even for off-line US segmentation the requirement for human supervision and interaction precludes full automation. To eliminate user interference and to speed up processing for both real-time and off-line applications, we developed an algorithm for the automatic artery recognition in longitudinal US scans of the CCA. It acts directly on the envelopes of received radio frequency echo signals, eventually composing the ultrasound image. In order to properly exploit the information content of the arterial structure the envelopes are decimated, according to the 2-dimensional resolution characteristics of the echo system, thereby substantially decreasing computational load. Subsequently, based upon the expected diameter range and a priori knowledge of the typical pattern in the echo envelope of the arterial wall-lumen complex, parametrical template matching is performed, resulting in the location of the lumen position along each echo line considered. Finally, in order to reject incorrect estimates, a spatial and temporal clustering method is applied. Adequate values for the parameters involved in the processing are obtained via off-line testing of the proposed algorithm on 128 echo data recordings from 45 subjects. Using those robust parameter values, correct and fast recognition of the artery is achieved in more than 98\% of the 6185 processed frames. Since these results are obtained via rigorous data decimation and using a cascade of rather simple steps, the proposed automatic algorithm is suitable for real-time recognition of the CCA.

\section{2 - Introduction}

Vascular non-invasive ultrasound (US) allows the estimation of morphological and dynamic parameters of arteries, such as diameter and distension (Reneman 2005) or intima-media thickness (IMT) (Van Bortel 2001). To perform these measurements quickly, accurately and reliably with minimal inter- and intra-user variability, the segmentation of the underlying ultrasound image should be computerized.

Image segmentation may be thought as consisting of two related processes (Udupa 2006): recognition, i.e. the high level task of determining roughly where a specific structure is located, and delineation, i.e. the low level task of determining the pre- 
cise spatial extent of such structure. The recognition task is better performed by the human eye rather than by any automatic algorithm, due to the capability of the human eye-brain system to integrate previous knowledge about objects during the recognition process ( $\mathrm{Wu}$ 2005). That is why in medical image segmentation the operator usually performs the recognition of the structure of interest manually, by placing seed points or an initialized dynamic curve in the targeted organ, or by cropping a region of interest (ROI) around it. After this a computer algorithm can perform the delineation automatically (Baillard 2000), being more accurate and precise than human observation.

When considering segmentation of ultrasound images, the automation of the whole process is complicated by a particular kind of image noise, i.e. the speckle pattern. Such noise originates from phase interference of ultrasound reflections by structures spaced at distances smaller than the resolution of the system in either direction (Burckhardt 1978). Speckle is responsible for two typical characteristics of ultrasound images. First of all, the grey level histogram in an ultrasound scan follows a statistic that, though being differently modelled in different acquiring conditions (Wagner 1983; Dutt 1996; Tao 2006), has as main features an asymmetric distribution and a correlation between its mean and variance. This makes the application of region-based clustering algorithms, such as the K-means, difficult for US segmentation purposes (Ashton 1995). Secondly, ultrasonic images have a typical grainy appearance where the aspect of continuous edges is ragged, low contrasted and blurred. Thus edge-based segmentation methods, such as Canny edge detection or Prewitt operators, are not suited to find boundaries between tissues (Davignon 2005). The presence of speckle in ultrasound images also causes conventional active contour models to fail, due to various local minima present in gradient-based energy functions, making it necessary to specifically modify the methods (Tao 2003). Both recognition and delineation processes are therefore not trivial in ultrasound image analysis.

In vascular ultrasound, various methods of contour detection are proposed in order to delineate the internal or external layers of arteries and to track their position over time. For non-invasive ultrasound, these measurements can be performed in M-Mode (Hoeks 1997), conventional B-Mode (Fan L 2001; Schmidt-Trucksass 2001; Cheng 2002; Sonka 2002) or high frame-rate B-Mode (Meinders 2001a). Vessel delineation methods are also developed for intravascular ultrasound (IVUS) images (Zhang 1998), in which the obvious advantages from an image-processing point of view are the a priori knowledge about the position of the arterial lumen (i.e. no need for recognition) and the high resolution of IVUS catheterized systems (which yields higher accuracy and precision for the delineation process). However, noninvasive imaging techniques are necessary in order to bring US-based vascular mor- 
phology assessment to common clinical screening practice. At the present state of the art, these methods require manual recognition of an artery prior to automatic contour delineation. This is the "bottleneck", limiting the possibilities to have totally automated US measurements of vascular properties in real-time, considering that US imaging provides a real-time image stream as a feedback to the user. An on-line manual recognition procedure forces the operator to concentrate both on lumen localization (in order to track the arterial movements) and on steady probe positioning (in order to optimize the plane of observation and angle of incidence between US beam and arterial walls). Fully automated artery recognition prior to contour delineation would limit user-induced artifacts and would ease the measurement procedure. Even off-line automatic segmentation of vascular US images would be attractive since it speeds up the procedure and offers the possibility to automatically analyze huge amounts of US frames without the need of human supervision.

To our knowledge, few attempts were made so far in order to automatically recognize the position of an artery in B-Mode scans. Fan (2000) proposed, without providing specific results, a method for the automatic recognition of the brachial artery as initialization for a flow mediated vasodilatation measurement procedure. The initial assumption is that the artery should be horizontally oriented in the image and should occupy the whole frame width. The algorithm is based on variance analysis of the transversal projection of the ultrasound image. The recognition assumes, as main property of the arterial lumen, a low variance in grey level with respect to that of surrounding regions, associating it to low echogenicity. This is true when considering pixel neighbourhoods in an unprocessed US image, given its typical grey-level statistic. However, the performed horizontal projection has the disadvantage of smoothing the variance characteristic, but also the echo level distribution if the assumptions about the orientation and extent of the artery fail. Another method was proposed (Delsanto 2007) in the context of a user-independent arterial segmentation procedure. Such automatic ROI identification is based on low-pass filtering, thresholding to find the far adventitial wall, and localization of the lumen based on grey level intensity and variance analysis for each single pixel column. This method proofed to be effective for 116 US frames, but was misleaded by arterial calcifications and not suited for real-time implementation.

In this chapter we introduce a novel algorithm for the automatic recognition of the common carotid artery (CCA) in B-Mode longitudinal US scans. We follow a rigorous multi-step approach in order to optimally exploit US echo signals and therefore further facilitate real-time applications. The algorithm operates intermittently, i.e. it processes subsequent frames coming from the US data stream, but at a decimated frame rate. The analysis of a single US frame is limited to a subset of pixel columns, each one representing the envelope of an acquired echo signal. The processing of 
each selected envelope involves 2 subsequent steps, i.e. decimation and template matching. Each step utilizes specific processing parameters, some of which are related to the characteristics of the ultrasound system, while others stem from a priori anatomical knowledge about the carotid artery. Detected CCA positions in subsequent frame pairs are then appraised using parametric clustering to reject outliers. The algorithm is validated with an off-line performance evaluation, in a direct comparison with manually obtained results for a large number of US data recordings, acquired by various operators from 45 subjects having different clinical conditions. The evaluation provides an optimal set of parameters for the segmentation of the common carotid artery in adult humans. The application of the algorithm is therefore totally user-independent, without the need for the sonographer to tune the parameters to the recording. We focus on the CCA because it is likely to be only slightly bended or angulated, as opposed to sites such as the carotid bifurcation, internal carotid artery, carotid bulb or external carotid artery, which can present more irregular shapes in the US scans. Our choice is also governed by the "Mannheim Carotid Intima-Media Thickness Consensus" (Touboul 2007) which, among the possible sites for the measurement of IMT on the carotid artery (CCA, bulb and internal carotid artery), indicates the CCA as the most advantageous one in terms of accessibility, differentiation between IMT and plaques, and higher reproducibility due to less vessel tortuosity. The method described in this chapter is meant for US frames with a longitudinal section of a single blood vessel, e.g. the CCA. In fact, it is common clinical knowledge that the presence of a vein in front of an artery is very likely to produce mirroring artifacts (Kurtz 2008) that can affect US-based arterial diagnoses.

\section{3 - Materials and methods}

\subsection{1 - Echo scanner and data sets}

All the analyzed images contain longitudinal scans of the CCA, and are acquired using a commercial software-based US device for the real-time measurement of vessel wall properties (ART.LAB, Esaote Europe BV, Maastricht, The Netherlands). Such system is based on a tracking method (Brands 1997; Brands 1999), and is composed of a PC connected to the front-end of an echo scanner (ESAOTE Picus), equipped with a 128 elements $7.5 \mathrm{MHz}$ linear array probe (actual received center frequency equal to $6.2 \mathrm{MHz}$ ). The B-Mode frame rate is $30 \mathrm{fps}$, each frame covering a $35 \mathrm{~mm}$ deep and $40 \mathrm{~mm}$ wide region (Fig. 4.1). The acquisition results in data sets containing the digitized versions of both the radio frequency (RF) signals and their envelopes as produced by the US scanner. The ART.LAB system stores B-Mode frames as a sequence of matrices. Each matrix consists of 128 echo lines (i.e. enve- 
lope signals) oriented column-wise as a function of depth, spaced at $315 \mu \mathrm{m}$ in the horizontal direction. The digitized envelopes are as they appear in the scanner just before scan conversion, and therefore they already passed through noise level thresholding and logarithmic compression. They are sampled at a frequency of 16.667 $\mathrm{MHz}$, in order to allow the ART.LAB system to perform accurate diameter estimation after manual ROI positioning. Assuming a constant sound velocity in tissue of $1540 \mathrm{~m} / \mathrm{s}$, the sample distance along the depth axis (vertical direction) is 46.2 micrometers $(\mu \mathrm{m})$. The digitization of the compressed envelope is performed with a dynamic range of 8 bits. For the development and performance evaluation of the algorithm, a set of 128 ART.LAB scan files with a recording time of 6-8 seconds each is used, for a total of 6185 processed frames. These recordings are from 45 subjects with a wide spectrum of ages and clinical conditions, from healthy to poststroke, and came from different clinical and research centers. Various sonographers performed the acquisitions, with gain settings varying correspondingly.

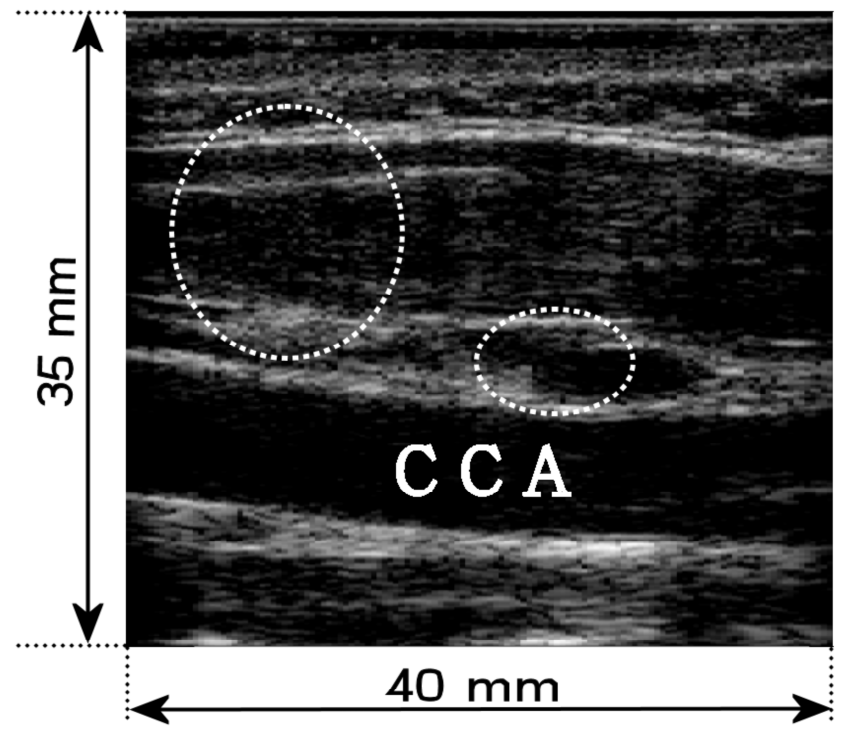

Fig. 4.1 - Ultrasound B-Mode image of the common carotid artery (CCA). In this frame, white ellipses indicate structures that locally mimic the pattern of a vessel. 


\subsection{2 - General scheme of the algorithm}

The general scheme with the overall structure of the algorithm is shown in Fig. 4.2. The main concept is that, for every processed frame, a finite subset of $\mathrm{N}$ equally spaced envelope signals is considered and resampled at a lower frequency (data decimation in both spatial directions). This concentrates the analysis on the information content of the artery, discarding particulars that are of no use for its localization. For each selected signal a lumen center position is estimated based on signal processing. The results for 2 subsequently analyzed frames are merged and subjected to a clustering method to discard outliers. Further data decimation is achieved by considering frames at a rate substantially lower than the acquisition and display frame rate. The algorithm is implemented and evaluated using MATLAB (Mathworks, Natick, MA).

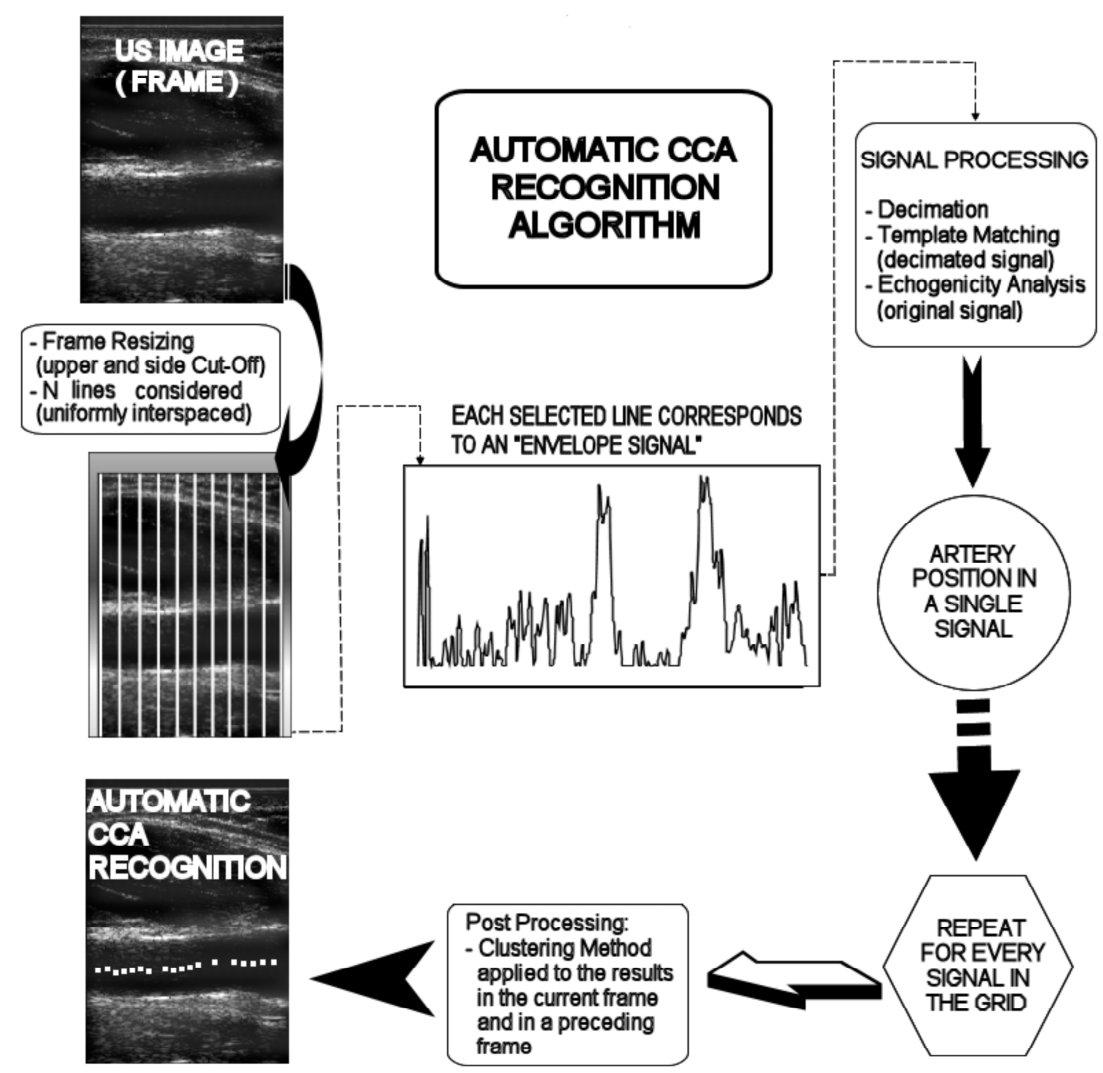

Fig. 4.2 - General scheme of the automatic CCA recognition algorithm, involving selection of envelope signals, signal processing and clustering. 


\subsection{3 - Selection of signals in the frames}

In US longitudinal scans, the CCA appears as a structure oriented in horizontal direction, often covering the entire frame width (Fig. 4.1). In the vertical dimension of the US frame, the artery is usually located at depths between 10 and $30 \mathrm{~mm}$ with a diameter between 4 and $9 \mathrm{~mm}$, depending on age and sex of the subject. The artery structure appears as a region having very low echogenicity (the lumen) surrounded by two bright bands (the arterial walls). The lumen center position may vary over the frame, consistent with a curved or inclined artery or with local narrowings of the lumen due to a stenosis. A vascular US image may contain many patterns mimicking the appearance of an artery of interest, e.g. actual tissue structures, artifacts, reverberations and other vessels (Fig. 4.1). The upper part of each frame (the first 2 $3 \mathrm{~mm}$ ) contains particulars that are of no interest for the lumen localization task (the gel-skin interface for instance). Moreover, users often tend to have the region of interest in the center of the image. Therefore, we ignore the information contained in the upper $3 \mathrm{~mm}$ and in the lateral $4 \mathrm{~mm}$ on each side $(10 \%$ of the image width). Within the resized frame, $\mathrm{N}$ equally spaced envelope signals are selected for further processing. For $\mathrm{N}=10$ the lateral interspacing between the selected envelope signals is about $3.5 \mathrm{~mm}$ while the lateral resolution of the US system ranges between 1.5 and $2 \mathrm{~mm}$. Hence, the frame decimation in the lateral direction is at a period of about twice the lateral resolution (Fig. 4.3a).

a)

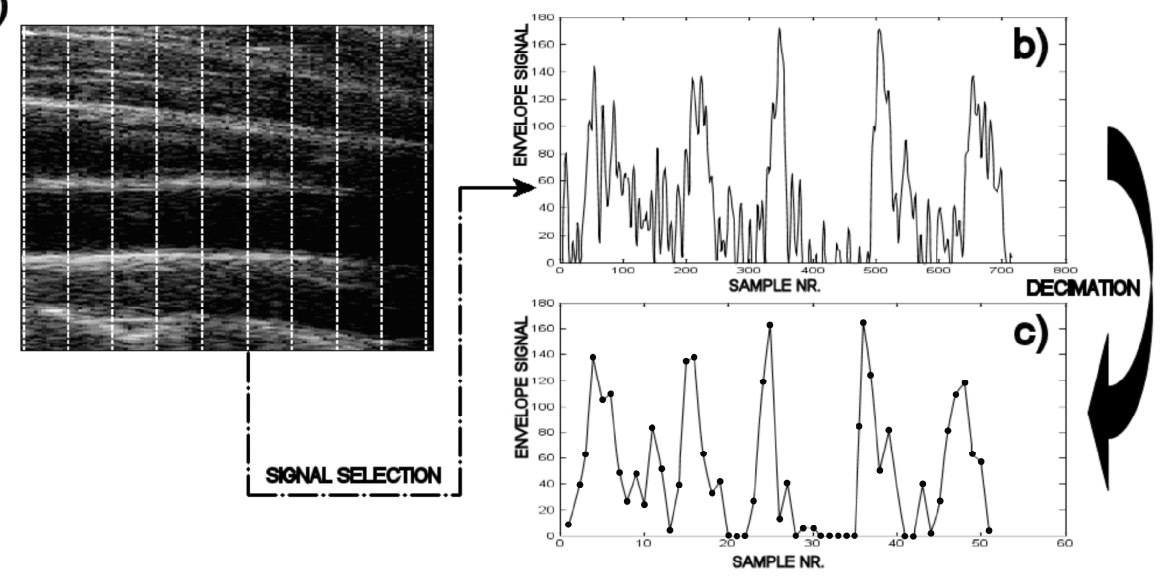

Fig. $4.3-a)$ in the B-mode US frame, the dashed white grid lines represent the selected envelope signals for further processing; b) the sampling frequency of an envelope signal is 16.667 $\mathrm{MHz}$ (intersample distance of $46.2 \mu \mathrm{m}$ ), and the number of samples (after the upper cut-off in the frame) equals $714 ; c$ ) the envelope signal after decimation: in this example the decimation period is $660 \mu \mathrm{m}$, i.e. twice the axial resolution of the US scanner used, resulting in a considerable reduction of the amount of samples. 


\subsection{4 - Signal decimation}

Fig. 4.3b shows an envelope signal, sampled at $16.667 \mathrm{MHz}$, corresponding to the $6^{\text {th }}$ selected line from the left in Fig. 4.3a. The artery structure appears as a large peak (near wall) approximately at sample number 350 , followed by a low amplitude region (blood) and a second large peak (far wall). The echo of a single reflector, as observed with ART.LAB system, has a duration of about 2.6 periods. For a center frequency of $6.2 \mathrm{MHz}$ (a wavelength of $248 \mu \mathrm{m}$ ), this converts to a system resolution of $330 \mu \mathrm{m}$. Depending on gain settings and tissue echogenicity, the width of the peaks in the envelope signal due to artery walls ranges from 0.5 to $1 \mathrm{~mm}$. Hence, the envelope signal can be safely decimated to a sampling distance of 1 or 2 times the resolution. This retains the information useful for the artery recognition purpose and considerably diminishes the amount of data to be processed. Fig. $4.3 \mathrm{c}$ shows an example of a decimated signal with a sample distance of $660 \mu \mathrm{m}$, i.e. twice the resolution of the used US scanner. Even after strong decimation the walllumen-wall pattern remains well recognizable.

\subsection{5 - Template matching}

The lumen features are its steep descent and ascent, separated by a segment with relatively low echo level. In the decimated envelope signal $\left(E_{D}\right)$ the edges can be approximated as rectilinear. Two separate templates are therefore generated, representing the left $\left(T_{L}\right)$ and right $\left(T_{R}\right)$ side of the artery structure, as indicated in Fig. 4.4. The parameters describing these templates are the widths of both their steep parts and their flat parts, which are once again expressed in units of depth resolution. The templates are scaled on the maximum of the decimated signal considered. The coarse nature of the templates matches the coarseness of the decimated signals, making it unnecessary to perform low pass filtering prior to template matching. Each template is swept over the decimated signal to calculate a depthdependent cost function CF1, defined as the sum of the squared differences between template and the underlying signal. Denoting the length of either template with $L t$ and its depth position with $d$, we have:

$$
C F 1(d)=\sum_{k=1}^{L t}\left[T_{X}(k)-E_{D}(d-1+k)\right]^{2}
$$

In (1), $T_{X}$ can mean either $T_{L}$ or $T_{R}$. For both $T_{L}$ and $T_{R}$, the positions of the four lowest values of CF1 are stored (Fig. 4.5). This is in order for each template to be able to detect the arterial wall also in the cases in which it is not associated to the absolute 


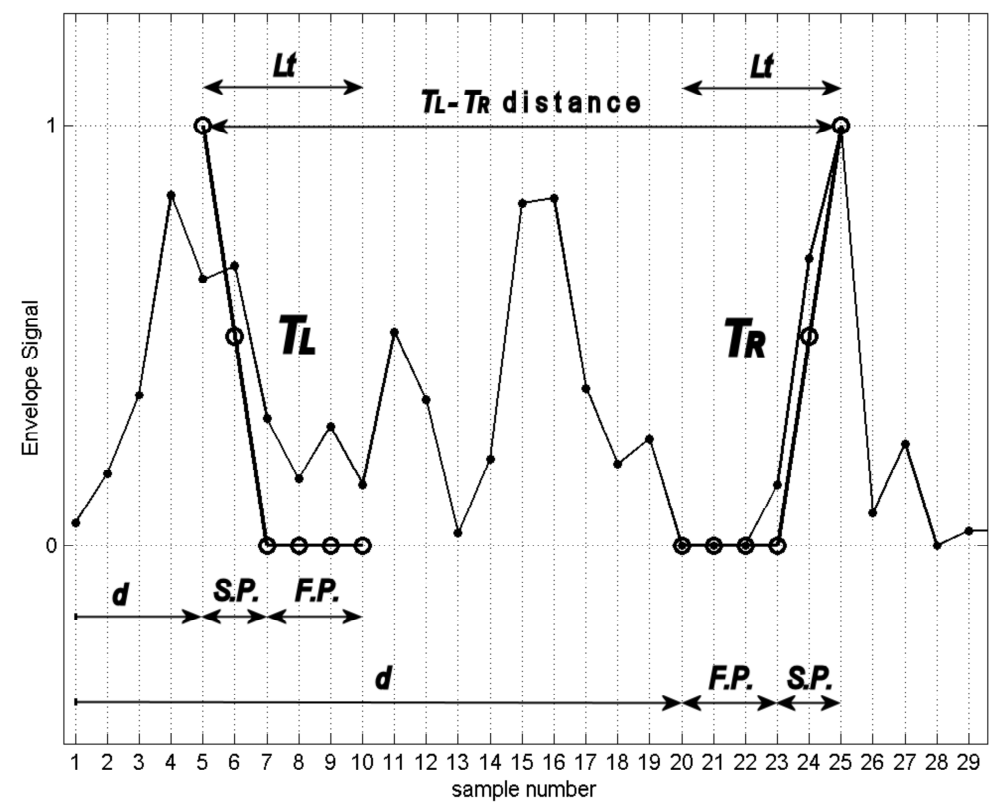

Fig. 4.4 - An example of the left (TL) and right template (TR) applied on a segment of the decimated signal. Each one of them consists of a steep (S.P.) and a flat part (F.P.). Template lengths in number of samples are denoted with $L t$, whereas depth position is denoted with $d$.

minimum of CF1. A first selection of possible lumen positions is made applying the constraint that the distance between the left and right arterial walls, identified by $T_{L}$ and $T_{R}$, must be in the typical CCA diameter range of adults. Such range is defined with two preset parameters, maximum and minimum allowed diameter. Subsequently every $T_{L}-T_{R}$ couple satisfying the geometrical constraint is re-mapped in the original envelope signal (Fig. 4.5) and a second cost function (CF2) is computed for every local envelope segment $\left(E_{L}\right)$ between both remapped templates. CF2 is based on the average of the squared derivatives with respect to depth $d$ and serves as an index of echogenicity because the envelopes of US echoes generally follow a distribution in which the average value and variation of the signal are positively correlated (Burckhardt 1978).

$$
C F 2(n)=\frac{1}{N L} \sum_{k=1}^{N L}\left[\frac{\partial\left(E_{L, n}\right)}{\partial d}(k)\right]^{2} n=1, \ldots, N T
$$

In equation (4.2), NT is the number of potential lumens identified in the signal according to the applied geometrical constraints. Each one of them relates to a differ- 
ent $\mathrm{E}_{\mathrm{L}}$ segment (identified by the variable $n$ ) with a different number $N L$ of samples. The middle point of the $\mathrm{E}_{\mathrm{L}}$ segment with the lowest CF2 is considered the center lumen position (Fig. 4.5).
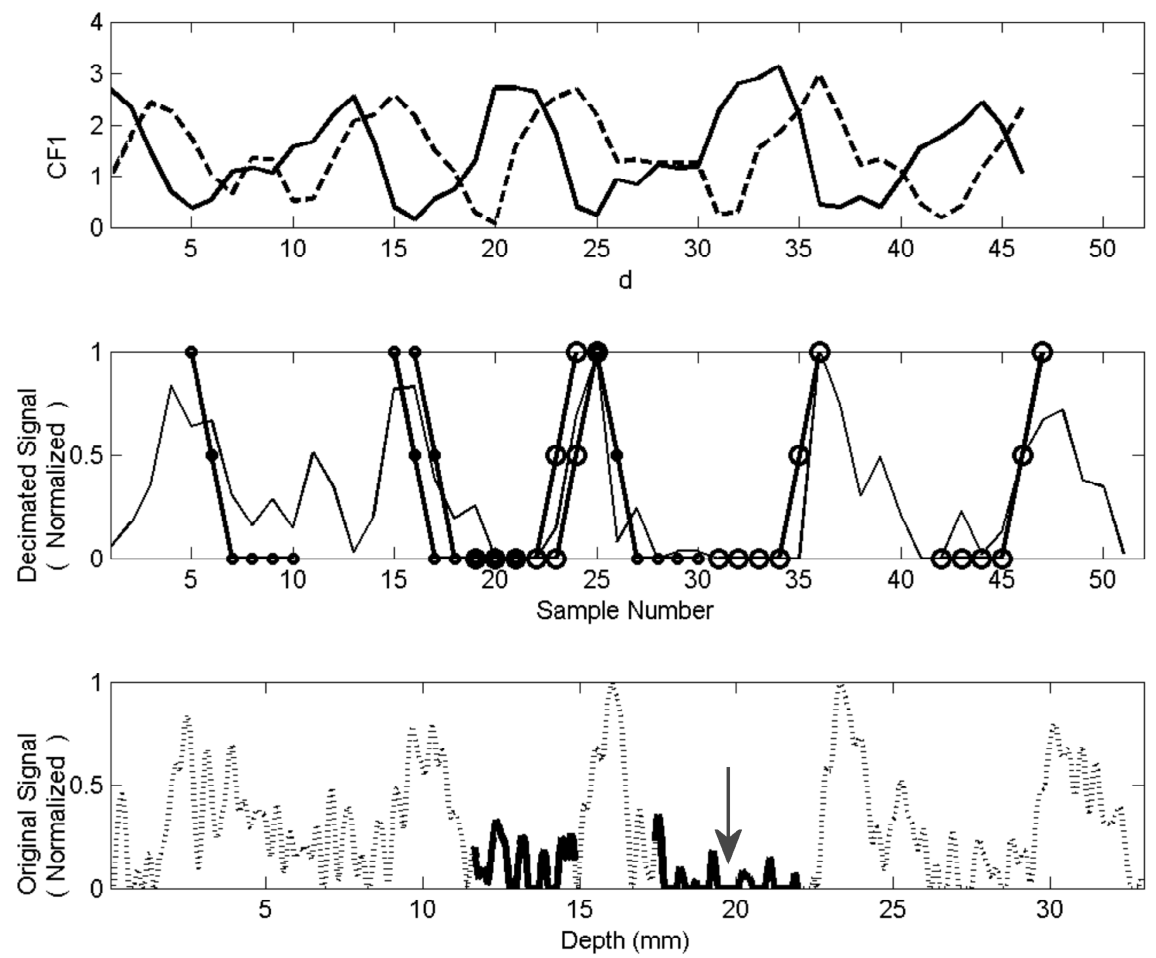

Fig. 4.5 - The upper graph represents the calculated cost function (CF1) as function of depth for the left (solid line) and right (dashed line) template. In the middle graph the template positions corresponding to the four lowest values of each CF1 track are shown. In the bottom graph the solid envelope segments represent possible lumen positions, resulting from the application of the geometrical constraints to the templates. Finally, application of a second cost function (CF2) yields the artery lumen center (black arrow) in the current signal.

\subsection{6 - Extending the algorithm in width and time: clustering}

As described so far, the algorithm exploits only the information regarding depth, but not width and time. After template matching, the success rate is between $60 \%$ and $80 \%$, depending on the settings (Table 4.4). This could be due either to artifacts in some signals or to the impossibility to find template matches meeting the imposed geometrical constraints. The CCA is a structure that can be slightly inclined and curved within a frame, implying variations in the lumen center position over the frame width. However, it may be assumed that these variations in position are small 
compared to the whole frame depth, as well as the local variations in lumen diameter.

Moreover, the overall displacement of the CCA in one cardiac cycle, caused by movements due to blood pressure variation or to slow hand motion, is low compared to the arterial diameter. The low variations in dimension and position within echo frames facilitate clustering of individual signal-based lumen center estimates, using the following steps:

1) Consider US frames at a rate lower than the acquisition frame rate, e.g. $6 \mathrm{fps}$.

2) Shift the signal grid in subsequently processed frames over half the grid line spacing, resulting in alternating odd and even numbers of gridlines per frame.

3) Merge detections from two subsequently analyzed frames (running mode) and sorting them in a depth-wise fashion. This reduces the effective lateral decimation period to one lateral resolution unit (in case $\mathrm{N}=10$ ).

4) Discriminate true lumen estimates from outliers based on consistency in the relative depth of lumen center positions.
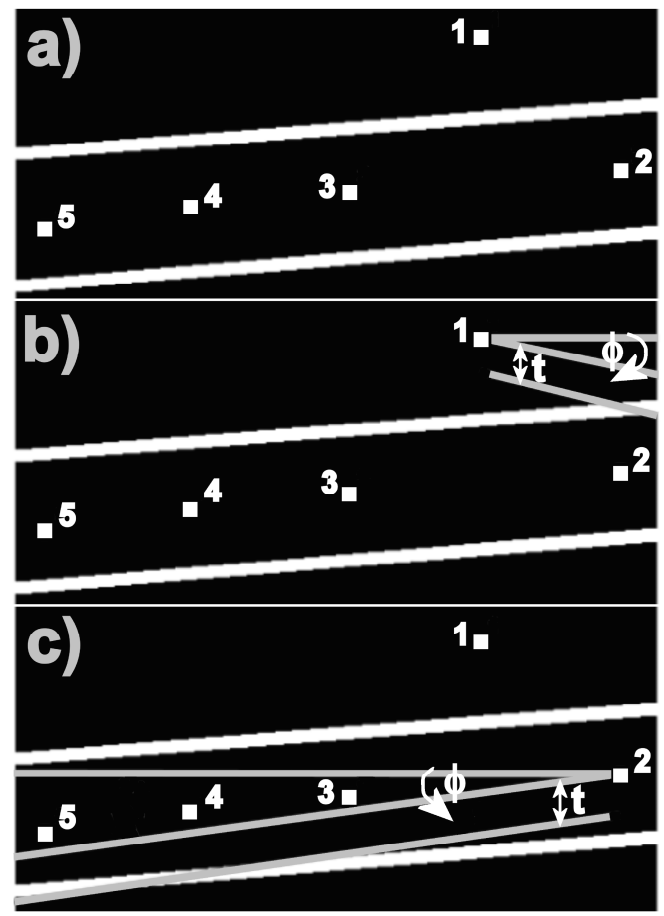

Fig. 4.6 - Schematic representation of the clustering procedure. Position \# 2 is not in the same cluster as position \# 1, but is in the same cluster as position \# 3 . At the end of the analysis, the largest cluster (in this example containing positions \# 2, 3, 4 and 5) refers to the arterial lumen. 
a)

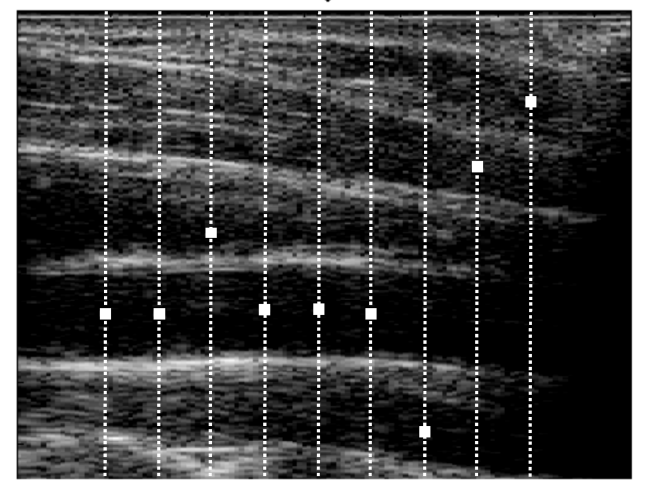

b)

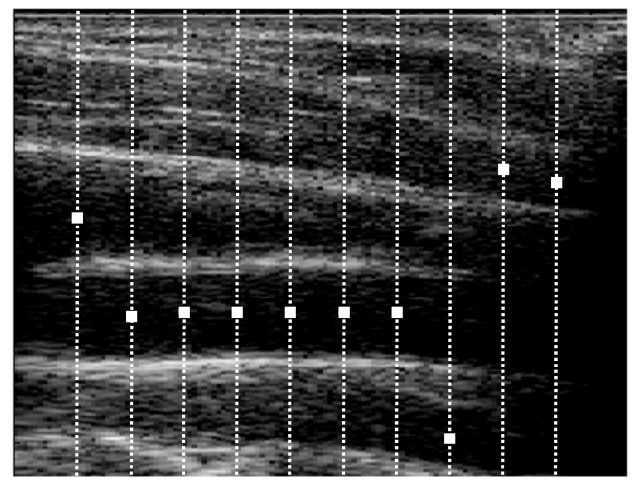

c)

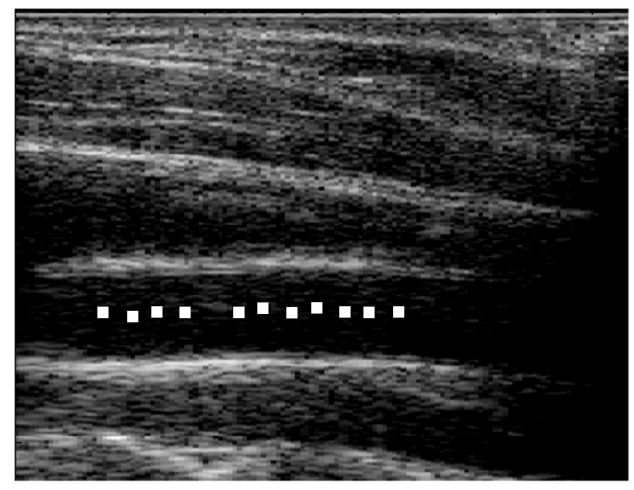

Fig. 4.7 - With an acquisition frame rate of $30 \mathrm{fps}$ and a processing rate of $6 \mathrm{fps}$ the skip factor between subsequently analyzed frames is 5 ; a) results in frame \#1 after template matching, with a shifted grid; b) results in frame \#6 after template matching; c) final result after clustering. Note that the algorithm correctly removes detection points in the right part of the frame, where an extensive black zone is present. The results shown are obtained with the optimal parameter set proposed in section 4.5. 
Fig. 4.6 presents a schematic explanation of the clustering procedure. The white inclined bands represent the vessel walls while the white dots are the identified lumen positions for a merged grid of 5 signals and numbered in ascending order of depth. Two independent parameters rule the clustering of subsequent lumen positions in the sorted list: an inclination tolerance $\varphi$, expressed in degrees, and an additional depth tolerance $t$ in units of resolution. The clustering mechanism starts with the first detected (most superficial) position in the list and checks if the depth of the next one complies with both clustering constraints. If the check is positive, position \#2 will be accepted in the same cluster as position \#1, otherwise position \#2 will be assigned to a new cluster (as in fact happens in this example). The same analysis is then repeated for every subsequent position in the sorted list. The final step of the clustering procedure is to retain the cluster with the highest number of positions. In this example the merged grid contains only 5 points. Figs. $4.7 \mathrm{a}$ and $4.7 \mathrm{~b}$ show the results of signal processing (decimation, CF1, CF2) on a total of 19 interlaced grid lines over two frames, while Fig. 4.7c shows the final CCA recognition obtained by merging and clustering the results.

\section{4 - Performance evaluation}

Table 4.1 - Constants in the evaluation

\begin{tabular}{ll}
\hline \hline Parameter & Value \\
\hline Upper cut-off & $3 \mathrm{~mm}$ (i.e. 65 samples) \\
Side cut-off & $4 \mathrm{~mm}$ (i.e. 13 echo lines at each side) \\
Max CCA diameter & $10.5 \mathrm{~mm}$ \\
Min CCA diameter & $5 \mathrm{~mm}$ \\
Frame-processing rate & $6 \mathrm{fps}$ \\
\hline \hline
\end{tabular}

Parameters kept constant in the performance evaluation of the algorithm. Upper and side cutoffs pertain to frame resizing, whereas maximum and minimum CCA diameters identify the typical range of diameter values in adults (adventitia - adventitia diameter).

In order to validate the algorithm, and also to arrive at an optimal set of values for the parameters involved in each step, a performance evaluation was carried out by comparing the results of the automatic CCA recognition with semi-automatically retrieved lumen center positions (reference position) for each processed frame in 128 echo recordings of a few seconds. The semi-automatic retrieval was performed with the ART.LAB system, in which the user first identifies the raw lumen position manually and then the automatic wall contour detection of the ART.LAB system results in a delineation of the arterial centerline. The automatic recognition performed by our proposed algorithm was considered correct if it did not deviate more 
than $2 \mathrm{~mm}$ from the reference position. A $2 \mathrm{~mm}$ acceptance range is consistent with the concept of recognition: the algorithm is designed to find the whereabouts of the CCA in the frame. Therefore such a wide range is adequate to evaluate automatically placed seed points for subsequent contour delineation algorithms. Values for the parameters that were kept constant throughout the evaluation, and are part of the optimal set we propose, are listed in Table 4.1. The total number of processed frames was 6185 .

The permitted CCA diameter range ( $5-10.5 \mathrm{~mm}$, see Table 4.1), used as geometrical constraint for the template matching during the evaluation, refers to the distance between the outermost samples in the templates (Fig. 4.4), representing peaks in the adventitial reflections, and thus to the external diameter of the artery. This means that the permitted inner lumen diameter can approximately range between 4 and $9.5 \mathrm{~mm}$, i.e. values that are well covering the CCA diameter range in adults. Such wide fixed range allows the template matching to cope with local stenoses or enlargements in the CCA. The preselected diameter range is the same for all results provided in this chapter. Parameters related to the clustering procedure were varied as listed in Table 4.2 (one depth resolution unit corresponds to $330 \mu \mathrm{m})$.

Table 4.2 - Clustering settings

\begin{tabular}{|c|c|c|c|c|}
\hline Color & Label & $\varphi$ (degrees) & t (resolution units) & Grid Lines $\mathrm{Nr}$. \\
\hline & A & 3 & 1 & 9 \\
\hline & B & 3 & 1 & 19 \\
\hline & C & 3 & 2 & 9 \\
\hline & D & 3 & 2 & 19 \\
\hline & $\mathrm{E}$ & 9 & 1 & 9 \\
\hline$\|$ & $\mathrm{F}$ & 9 & 1 & 19 \\
\hline \#曲 & G & 9 & 2 & 9 \\
\hline 事 & $\mathrm{H}$ & 9 & 2 & 19 \\
\hline
\end{tabular}

Parameter combinations for clustering in the performance evaluation. The last column gives the total number of grid lines over two subsequently processed frames considered for clustering. Colors refer to box-whiskers plots in Fig. 4.8.

For each combination listed in Table 4.2, parameters related to decimation and template dimensions were varied as shown in Table 4.3. The decimation period varies between one and two units of resolution, whereas there are two size options for the templates, type $\mathrm{N}$ (narrow) and type $\mathrm{W}$ (wide). It is worth noting that tem- 
plate type $\mathrm{W}$ in the case of decimation to 1 resolution unit has the same dimensions as template type $\mathrm{N}$ for a decimation to 2 resolution units. For each possible parameter combination, four performance evaluators were considered to rate the results of the automatic CCA recognition. The Initial Hit Rate (Table 4.4) indicates the average success percentage for all considered frames before applying clustering. As already mentioned, the clustering process will retain the most populated cluster: the number of detected points in such cluster is evaluated for each merged frame as the percentage of the total number of grid lines considered. Its average over all processed frames is indicated by Clustered Positions (Table 4.4). The Final Hit Rate (Fig. 4.8) expresses the percentage of correct lumen estimates within the accepted cluster, averaged over all merged frames.

Table 4.3 - Template definitions

\begin{tabular}{l}
\hline Template type: $\mathbf{N}$ \\
$\begin{array}{l}\text { Decimation: } 1 \text { resolution unit. } \\
\text { Intersample distance }=330 \mu \mathrm{m}\end{array}$
\end{tabular}

Decimation: 2 resolution units. Intersample distance $=660 \mu \mathrm{m}$
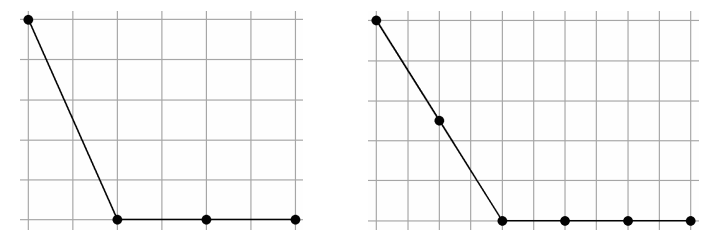

Parameter combinations for signal decimation and template dimensions in units of depth resolution. For each of the two decimation rates used, a narrow $(\mathrm{N})$ and a wide $(\mathrm{W})$ template are considered.

The average Frame Coverage (Table 4.4) is expressed by the ratio of the distance between the outermost echo signals with a correctly identified lumen position and the resized frame width. Clustered Positions and Frame Coverage show how the algorithm is able to properly cover the artery over the frame width.

The most significant output parameter is the Final Hit Rate because it is directly related to the amount of correct recognitions. The results are shown in boxwhiskers plots (Fig. 4.8). The first order statistics (average and standard deviation) of the other performance evaluators are shown in Table 4.4. Fig. 4.9 shows some automatic lumen recognition examples. Table 4.5 lists the average values of the 
Final Hit Rate both for the default case of logarithmically compressed signals (columns denoted by " $\mathrm{C}$ ", showing the values of the black filled circles in Fig. 4.8) and also, to show the effect of compression, for non-compressed signals (columns denoted with "NC").

On a general purpose PC (Intel Celeron $3 \mathrm{GHz}, 448 \mathrm{MB}$ of RAM), the average time required in order to process one frame, including data loading, is in the order of 200 milliseconds (MATLAB implementation). This demonstrates that the algorithm is suited for real-time intermittent recognition of the CCA.

Table 4.4 - Preliminary performance evaluation

\begin{tabular}{|c|c|c|c|c|c|c|c|c|}
\hline \multirow{4}{*}{$\begin{array}{l}\text { INITIAL } \\
\text { HIT RATE [\%] } \\
\begin{array}{c}\text { CLUSTERING } \\
\text { SETTINGS } \\
\text { GROUP }\end{array}\end{array}$} & \multicolumn{8}{|c|}{ DECIMATION AND TEMPLATE MATCHING PARAMETERS } \\
\hline & \multicolumn{4}{|c|}{ Decimation: 1 Resolution Unit } & \multicolumn{4}{|c|}{ Decimation: 2 Resolution Units } \\
\hline & \multicolumn{2}{|c|}{ Template $\mathrm{N}$} & \multicolumn{2}{|c|}{ Template W } & \multicolumn{2}{|c|}{ Template $\mathrm{N}$} & \multicolumn{2}{|c|}{ Template W } \\
\hline & AVERAGE & SD & AVERAGE & SD & AVERAGE & SD & AVERAGE & SD \\
\hline $\mathrm{A}$ & 61.0 & 15.3 & 74.0 & 16.5 & 72.6 & 15.3 & 74.4 & 16.3 \\
\hline B & 65.2 & 14.3 & 78.9 & 14.3 & 78.0 & 13.8 & 79.9 & 14.4 \\
\hline $\mathrm{C}$ & 65.8 & 14.8 & 80.1 & 14.3 & 78.9 & 13.2 & 80.6 & 14.4 \\
\hline D & 69.0 & 13.6 & 83.1 & 12.7 & 81.7 & 12.6 & 83.6 & 13.1 \\
\hline $\mathrm{E}$ & 67.3 & 14.4 & 81.6 & 13.5 & 79.7 & 12.5 & 81.4 & 13.9 \\
\hline $\mathrm{F}$ & 69.1 & 13.6 & 83.1 & 12.4 & 80.9 & 12.7 & 82.9 & 13.0 \\
\hline G & 68.0 & 14.5 & 82.6 & 13.2 & 81.1 & 12.4 & 82.9 & 13.7 \\
\hline H & 69.9 & 13.4 & 83.8 & 12.2 & 82.5 & 12.1 & 84.2 & 12.7 \\
\hline \multirow{3}{*}{$\begin{array}{l}\text { CLUSTERED } \\
\text { POSITIONS [\%] }\end{array}$} & \multicolumn{8}{|c|}{ DECIMATION AND TEMPLATE MATCHING PARAMETERS } \\
\hline & \multicolumn{4}{|c|}{ Decimation: 1 Resolution Unit } & \multicolumn{4}{|c|}{ Decimation: 2 Resolution Units } \\
\hline & \multicolumn{2}{|c|}{ Template $\mathrm{N}$} & \multicolumn{2}{|c|}{ Template W } & \multicolumn{2}{|c|}{ Template $\mathrm{N}$} & \multicolumn{2}{|c|}{ Template W } \\
\hline $\begin{array}{l}\text { CLUSTERING } \\
\text { SETTINGS } \\
\text { GROUP }\end{array}$ & AVERAGE & SD & AVERAGE & SD & AVERAGE & SD & AVERAGE & SD \\
\hline $\mathrm{A}$ & 63.0 & 12.7 & 74.9 & 14.8 & 73.4 & 14.3 & 75.4 & 14.8 \\
\hline B & 66.2 & 12.8 & 79.4 & 13.3 & 78.6 & 12.6 & 80.6 & 13.2 \\
\hline $\mathrm{C}$ & 68.1 & 12.0 & 80.9 & 12.6 & 79.7 & 12.1 & 81.7 & 12.5 \\
\hline D & 70.2 & 12.2 & 83.8 & 11.7 & 82.7 & 11.0 & 84.6 & 11.7 \\
\hline $\mathrm{E}$ & 70.6 & 11.1 & 82.9 & 11.6 & 81.2 & 11.1 & 82.9 & 11.6 \\
\hline $\mathrm{F}$ & 71.0 & 11.7 & 84.1 & 11.2 & 82.2 & 10.8 & 84.1 & 11.4 \\
\hline G & 71.8 & 11.1 & 84.1 & 11.2 & 82.9 & 10.8 & 84.8 & 11.1 \\
\hline H & 72.1 & 11.3 & 85.0 & 11.0 & 84.2 & 10.1 & 85.8 & 11.0 \\
\hline \multirow{3}{*}{$\begin{array}{l}\text { FRAME } \\
\text { COVERAGE [\%] }\end{array}$} & \multicolumn{8}{|c|}{ DECIMATION AND TEMPLATE MATCHING PARAMETERS } \\
\hline & \multicolumn{4}{|c|}{ Decimation: 1 Resolution Unit } & \multicolumn{4}{|c|}{ Decimation: 2 Resolution Units } \\
\hline & \multicolumn{2}{|c|}{ Template N } & \multicolumn{2}{|c|}{ Template W } & \multicolumn{2}{|c|}{ Template $\mathrm{N}$} & \multicolumn{2}{|c|}{ Template W } \\
\hline $\begin{array}{l}\text { CLUSTERING } \\
\text { SETTINGS } \\
\text { GROUP }\end{array}$ & AVERAGE & SD & AVERAGE & SD & AVERAGE & SD & AVERAGE & SD \\
\hline A & 71.8 & 17.0 & 78.8 & 17.6 & 79.8 & 15.3 & 80.4 & 16.3 \\
\hline B & 83.0 & 12.7 & 87.3 & 11.7 & 88.1 & 11.2 & 88.6 & 11.4 \\
\hline $\mathrm{C}$ & 77.1 & 15.4 & 85.6 & 14.0 & 86.1 & 12.0 & 86.5 & 13.6 \\
\hline D & 87.1 & 10.5 & 91.6 & 9.1 & 91.5 & 9.5 & 92.0 & 9.5 \\
\hline $\mathrm{E}$ & 79.3 & 14.1 & 87.7 & 12.5 & 87.3 & 11.1 & 87.6 & 12.8 \\
\hline $\mathrm{F}$ & 87.5 & 10.1 & 91.8 & 8.5 & 91.0 & 9.5 & 91.6 & 9.3 \\
\hline G & 80.0 & 14.2 & 88.8 & 12.1 & 88.6 & 11.0 & 89.1 & 12.7 \\
\hline H & 88.3 & 9.7 & 92.4 & 8.4 & 92.3 & 9.0 & 92.6 & 9.2 \\
\hline
\end{tabular}

Averages and standard deviations (SD) refer to the analysis of 128 echo data sets. 

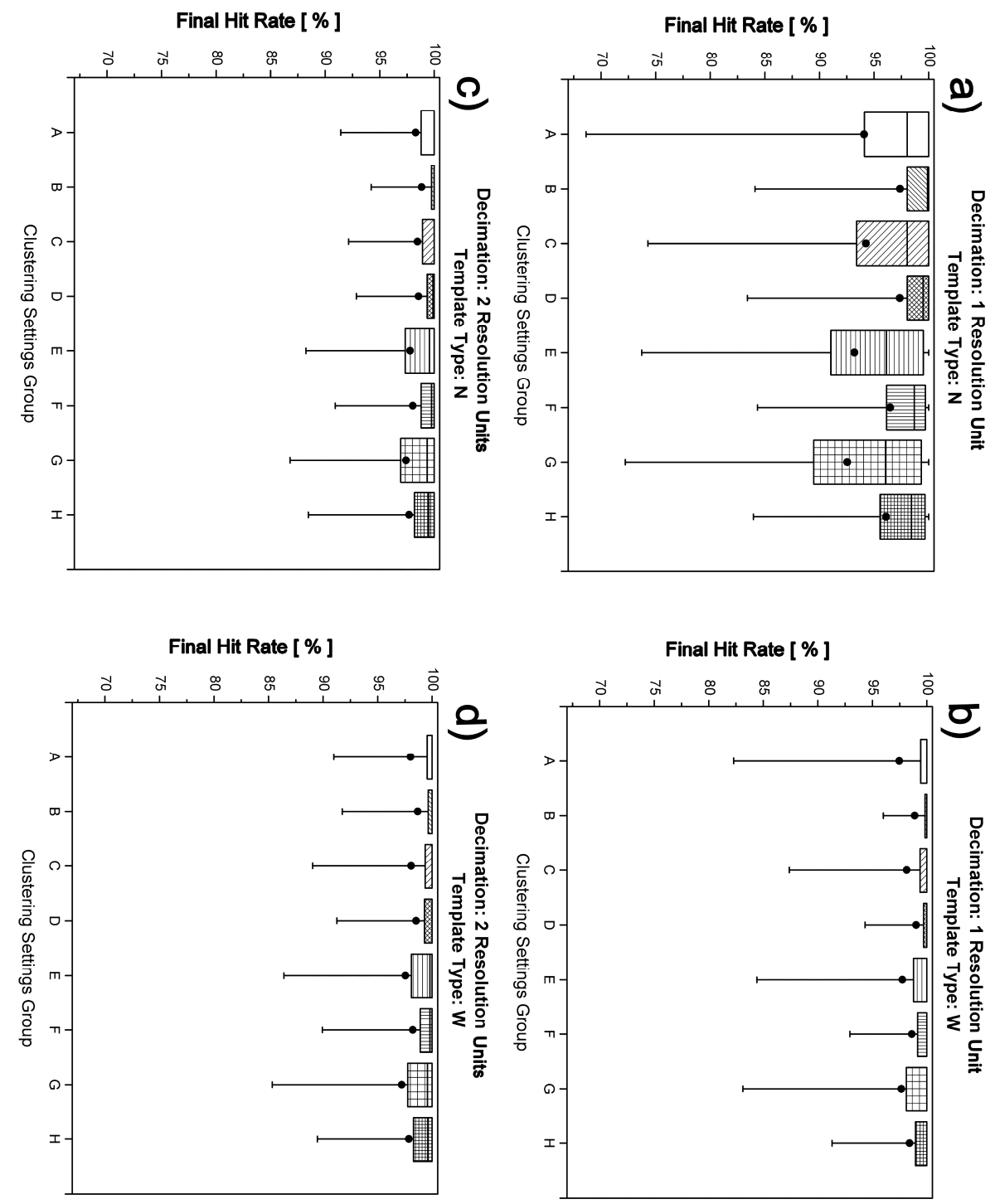

Fig. 4.8 - Box and whiskers plot showing distributions of the Final Hit Rate (over 128 US data sets) for all parameter combinations used in the performance evaluation. The box shows the 25th, 50th and 75th percentiles, while the whiskers cover the 5th and 95th percentile range. Black filled circles represent averages. 
a)

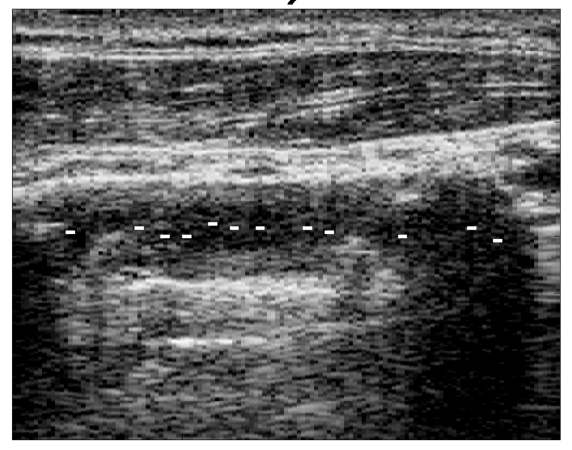

b)

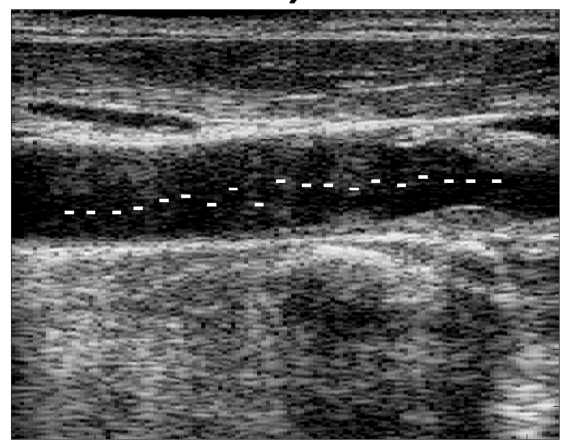

C)

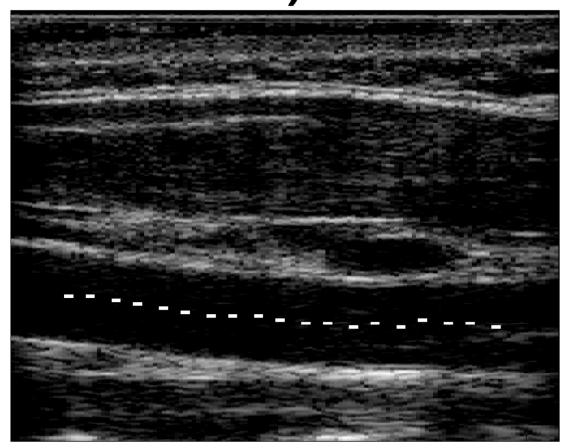

d)

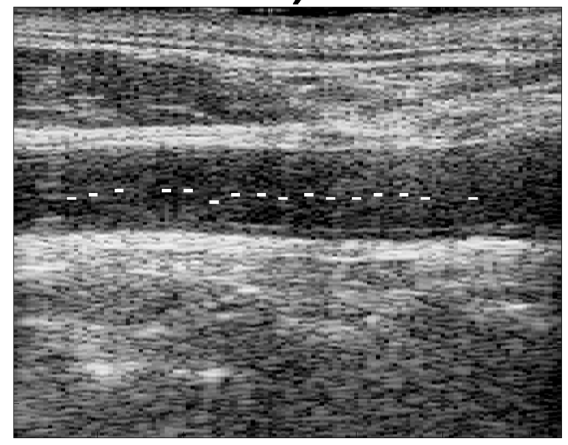

e)

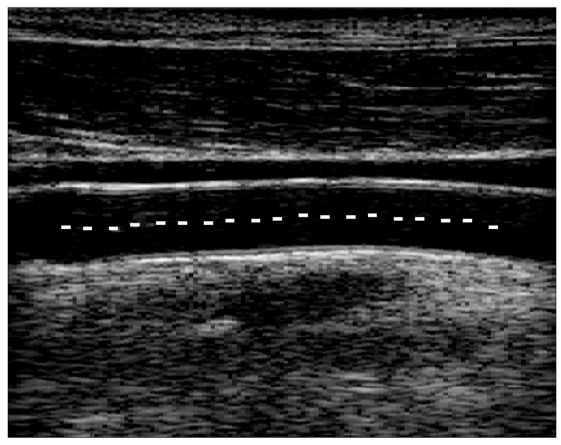

f)

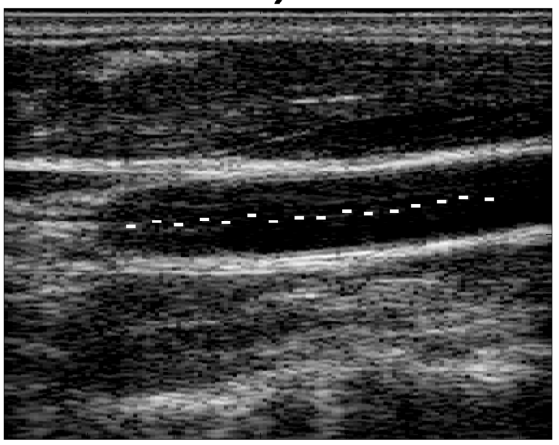

Fig. 4.9 - Examples of the proposed automatic CCA recognition algorithm; a), b) and d) show that the algorithm is effective also in the presence of atherosclerotic plaques and/or bad quality of the echo image; in c) and e) the artery is properly identified despite other structures mimicking an arterial pattern; f) presents a case in which the lumen is identified only in the part of the frame in which it is present. This set of images illustrates that the algorithm works properly for different inclinations, bending patterns, average depth of the CCA and average grey level, indicating that the method is robust and insensitive to US gain settings. All results shown are obtained using the same parameter set as proposed in section 4.5 , without the need to specifically tune the processing parameters. 
Table 4.5 - Final Hit Rate

\begin{tabular}{|c|c|c|c|c|c|c|c|c|}
\hline \multirow[b]{3}{*}{ Clustering } & \multicolumn{4}{|c|}{ Narrow Template } & \multicolumn{4}{|c|}{ Wide Template } \\
\hline & \multicolumn{2}{|l|}{$1 \mathrm{RU}$} & \multicolumn{2}{|l|}{$2 \mathrm{RU}$} & \multicolumn{2}{|l|}{$1 \mathrm{RU}$} & \multicolumn{2}{|l|}{$2 \mathrm{RU}$} \\
\hline & C & NC & C & NC & C & NC & C & NC \\
\hline A & 94.1 & 83.7 & 98.3 & 89.6 & 97.4 & 86.9 & 98.0 & 90.3 \\
\hline B & 97.4 & 89.4 & 98.8 & 93.9 & 98.9 & 91.6 & 98.7 & 94.4 \\
\hline C & 94.2 & 84.3 & 98.5 & 90.1 & 98.1 & 88.0 & 98.0 & 90.8 \\
\hline D & 97.4 & 89.1 & 98.5 & 93.3 & 99.0 & 91.5 & 98.5 & 94.2 \\
\hline $\mathbf{E}$ & 93.2 & 82.9 & 97.8 & 88.8 & 97.7 & 87.5 & 97.5 & 89.9 \\
\hline $\mathbf{F}$ & 96.5 & 88.3 & 98.0 & 92.5 & 98.6 & 91.0 & 98.2 & 93.6 \\
\hline G & 92.5 & 82.5 & 97.4 & 88.2 & 97.6 & 87.2 & 97.2 & 89.4 \\
\hline H & 96.1 & 87.6 & 97.7 & 91.8 & 98.4 & 90.4 & 97.9 & 93.0 \\
\hline
\end{tabular}

Average Final Hit Rate [\%] for both logarithmically compressed (C) and uncompressed (NC) envelopes for every considered parameter combination. The letters on the left refer to the clustering cases of Table 4.2. RU stands for "resolution units". The hit rate for uncompressed images is on average $7.8 \%$ lower than that of compressed ones.

\section{5 - Discussion}

In this chapter we introduce an algorithm for the automatic recognition of the common carotid artery in longitudinal ultrasound B-mode scans, based on a series of morphological operations. The strength of the algorithm lies in the combination (template matching, grey level variability assessment, clustering) rather than in the single techniques. The performance evaluation yields a fixed set of processing parameters adequate for automatic CCA recognition without any user interference. Moreover, the extensive data decimation performed allows the method to optimally exploit the information content of the artery and facilitates real-time operation.

In general our proposed automatic CCA recognition algorithm performs very well: in up to $98-99 \%$ (Table 4.5) of the considered images the artery position is correctly identified. An important step in the recognition process is the final clustering of the lumen estimates. Without clustering, the success rate did not exceed the $85 \%$ level (Table 4.4) although signal-processing parameters (decimation, template size and shape) were varied over a wide range. As expected, the Final Hit Rate distributions (Fig. 4.8) are very asymmetric with a mean value very close to the $25^{\text {th }}$ percentile, and the $75^{\text {th }}$ percentile almost always approaching the $100 \%$ value. Because the $25^{\text {th }}$ 
percentile is never lower than $88 \%$, it indicates a very good performance of the algorithm. As Fig. 4.8 and Table 4.5 show, Final Hit Rate averages are systematically higher for the case in which the clustering acts on 19 (horizontal decimation to 1 resolution) rather than on 9 grid points; thus a proper grid line spacing supports the clustering method to exploit relative consistencies across the image. It is interesting how the box-and-whiskers plots are on average narrower in the decimation to 2 resolution units rather than 1 resolution unit (Fig. $4.8 \mathrm{c}$ and $4.8 \mathrm{~d}$ ). This illustrates that a coarse approach using strong decimation locates the artery more efficiently. For the 2 resolution units decimation case, results are hardly different between template $\mathrm{N}$ and $\mathrm{W}$. This favors the choice of the $\mathrm{N}$ type, which involves a smaller number of samples per template and thus diminishes computational load. The values considered for the inclination angle $\varphi$ ( 3 or 9 degrees) may seem very small, but their possible drawback is just wrongful rejection of correct estimates (false negatives). On the other hand, larger values would result in a larger number of false positives. The fact that $\varphi$ and $t$ (depth tolerance) are independent from each other makes the clustering procedure very flexible. Fig. 4.8 shows that for CCA recognition an inclination angle $\varphi$ of 3 degrees gives generally better results in terms of Final Hit Rate distributions than a 9 degrees angle. Depth tolerance $t$ does not seem instead to influence the results once $\varphi$ is set. A consequence of small values for $\varphi$ and $t$ is that arteries with high inclination angles can be difficult to recognize. However, this is rarely the case for the CCA, for which the parameter set is designed.

Table 4.6 - Optimal parameter set for automatic CCA recognition

\begin{tabular}{ll}
\hline \hline Parameter & Optimal value for CCA \\
Max CCA diameter & $10.5 \mathrm{~mm}$ \\
Min CCA diameter & $5 \mathrm{~mm}$ \\
Frame-processing rate & $6 \mathrm{fps}$ \\
Decimation & 2 resolution units \\
Template type & $\mathrm{N}$ (Narrow) \\
$\varphi$ & 3 degrees \\
$t$ & 1 resolution unit \\
\hline \hline
\end{tabular}

Set of parameter values for the automatic recognition of the CCA in adults.

The above-mentioned considerations result in a very robust parameter set for the automatic CCA recognition (Table 4.6). For clarity the diameter range and the frame-processing rate are also included, even though they were kept constant in the evaluation. 
The Final Hit Rate distribution for the selected parameter set is very narrow with the $5^{\text {th }}$ percentile at about $94 \%$, the $25^{\text {th }}$ percentile above $99 \%$ (column B of Fig. 4.8c) and an average value of $98.8 \%$ (Table 4.5). The examples of Fig. 4.7 and Fig. 4.9 were obtained with these settings, demonstrating the high robustness of the proposed parameter set. Fig. 4.9 illustrates that proper recognition of the CCA is achieved despite poor image quality, irregular luminal diameter due to atherosclerotic plaques, or artery inclination and curvature. The detection capability is quite immune to variations in luminal grey level or in sharpness of edges representing the arterial walls. The algorithm adapts itself to the actual extension of the artery lumen within the frame, meaning that zones without an artery do not get recognition points (see Fig. 4.7). This is a very useful feature of the algorithm since it motivates sonographers to get scan planes with a better section of the CCA. In some cases the points within the lumen are quite sparse and not uniformly distributed over the frame width, but even then they univocally identify a ROI including the artery, which is the main purpose of the algorithm.

The algorithm exploits the fact that the lumen has low amplitude with respect to its environment, a known width range and a horizontal or slightly inclined orientation. With the selected parameter set, analysis of 128 data sets yielded only 6 data sets with results below $95 \%$. 4 of these were between $90 \%$ and $95 \%$ while 2 were below $90 \%$. The corresponding images showed that the lower performance was due to either a low signal to noise ratio in the lumen caused by reverberations, or to the presence of the jugular vein within the image. In the former case, a highly echogenic lumen misleads the CF1-based analysis, preventing the templates to find their proper positions across wall-lumen interfaces. On the other hand the simultaneous presence of the jugular vein, exhibiting similar characteristics to the CCA, can cause failure of the algorithm in two different ways. If the apparent thickness of the near wall of the CCA becomes too low due to the neighbouring jugular, the template can fail finding it. If the templates properly identify all the walls, CF2 and clustering may still favour the vein rather than the artery, although in most cases the algorithm gives preference to an artery because venous walls are less echogenic than arterial ones (CF1), the venous lumen exhibits a higher amplitude variation than the arterial lumen due to a sub-optimal scan plane (CF2), and the venous diameter is usually not constant across the frame due to the incident echo section or unintended compression. The algorithm has been designed for longitudinal US scans showing exclusively the CCA. From a technical point of view the extension of the recognizing capabilities to images with both CCA and jugular is an interesting future development. However, the total or partial presence of the jugular vein in front of the CCA considerably 
increases the risk of mirroring artifacts within the CCA (Kurtz 2008). This is undesirable from a clinical point of view, because such artifacts may lead to a false diagnosis, e.g. presence of carotid dissections, or to wrong estimates for CCA diameter and IMT.

In the present study, the evaluation was performed on image sequences with logarithmic amplitude compression, as is conventional in commercial US scanners to adapt the dynamic range for visualization purposes. In order to further explore the robustness of the proposed algorithm, the analysis was repeated for uncompressed envelopes of the same recordings. For this purpose, the RF echo signals rather than their (compressed) envelopes were captured at a sampling frequency of $33.3 \mathrm{MHz}$ and with an acquisition dynamic range of 12 bits. The off-line software computation of the envelopes from the RF signals involved band pass filtering (bandwidth of 3.1 $\mathrm{MHz}$ centered at $6.2 \mathrm{MHz}$ ), followed by time-domain discrete Hilbert transform and decimation by a factor of 2 . At this stage the envelope of the Hilbert pair has the same sample rate and is subsequently subjected to the same processing steps as applied to the logarithmic compressed signal. The Final Hit Rate for uncompressed envelopes had wider distributions and appeared to be on average $7.8 \%$ lower than for compressed signals (Table 4.5). The deviating behaviour could be due to the fact that for uncompressed envelopes differences in amplitude between black zones outside and inside the arterial lumen are less evident than for compressed ones. However, despite different preprocessing, the selected parameter set (Table 4.6) gives results in the highest range also in case of no logarithmic compression.

Processing all pixels within a B-Mode frame is not a well-suited approach for automatic CCA recognition, because it implies the analysis of a redundant amount of data far beyond the information content of the wall-lumen-wall structure in the US frame. Relying on signal processing of a finite subset of decimated echo lines, as in our case, removes redundancy and guarantees a higher efficiency, effectiveness and real-time suitability of the automatic CCA recognition procedure. Our results also suggest that the algorithm is very robust for different clinical conditions, different operators, different gain settings and different pre-processing of the envelope signals. This suggests that the method could be incorporated at any point of the US signal-processing chain, i.e. right after focusing, after logarithmic compression or even after scan conversion. Moreover, having decimation periods, template dimensions and depth tolerance expressed in units of axial and lateral resolution makes the algorithm portable to US systems with a different image structure, carrier frequency and bandwidth. 


\section{6- Conclusion}

We proposed an algorithm for the automatic recognition of the position of the common carotid artery in sequences of ultrasound images, based on signal decimation, template matching and clustering. The performance evaluation yields a robust parameter set that provides very reliable estimates, regardless of the preprocessing of the considered envelopes. The high performance (average success rate of $98.8 \%$ for logarithmic compressed signals) is independent of gain settings, sonographers, or clinical condition of subjects. Decimation of the US matrices according to the resolution of the ultrasound system and to the dynamic image characteristics (artery motion, transducer adjustments) reduces considerably the computational load, and will eventually allow real-time application of the algorithm, as demonstrated by the low amount of time required to process one frame. The algorithm is also quite insensitive to the effects of dynamic range compression.

The implementation of the proposed automatic arterial recognition in US systems would fully automate the segmentation of longitudinal B-Mode scans of the common carotid artery. This would enforce non-invasive arterial US analysis in clinical practice for screening, treatment monitoring, and in non-invasive estimation of arterial mechanical properties (Meinders 2001b; Pannier 2002; Meinders 2004; Reneman 2006; Paini 2007). Moreover, due to the parametric nature of the recognition process, the algorithm could be extended to brachial and femoral arteries, jugular veins, and other organs both in US and in other imaging modalities, by means of tuning the permitted diameter range or other parameters. This would bring medical image segmentation closer to full automation.

\section{References}

Ashton EA, Parker KJ. Multiple resolution Bayesian segmentation of ultrasound images. Ultrasonic Imaging 1995; 17: 291-304.

Baillard C, Barillot C, Robust 3D segmentation of anatomical structures with level sets. In: Medical Image Computing and Computer-Assisted Intervention - MICCAI 2000, Springer Berlin / Heideberg, 2000.

Brands PJ, Hoeks APG, Ledoux LAF, Reneman RS. A radio frequency domain complex cross-correlation model to estimate blood flow velocity and tissue motion by means of ultrasound. Ultrasound in Medicine \& Biology 1997; 23: 911-920.

Brands PJ, Hoeks APG, Willigers J, Willekes C, Reneman RS. An integrated system for the non-invasive assessment of vessel wall and hemodynamic properties of large arteries by means of ultrasound. European Journal of Ultrasound 1999; 9: 257-266. 
Burckhardt CB. Speckle in ultrasound B-Mode scans. IEEE Transactions On Sonics And Ultrasonics 1978; 25: 1-6.

Cheng D, Schmidt-Trucksass A, Cheng K, Burkhardt H. Using snakes to detect the intimal and adventitial layers of the common carotid artery wall in sonographic images. Computer Methods and Programs in Biomedicine 2002; 67: 27-37.

Davignon F, Deprez J-F, Basset O. A parametric imaging approach for the segmentation of ultrasound data. Ultrasonics 2005; 43: 789-801.

Delsanto S, Molinari F, Giusetto P, Liboni W, Badalamenti S, Suri JS. Characterization of a completely user-independent algorithm for carotid artery segmentation in 2-D ultrasound images. IEEE Transactions On Instrumentation And Measurement 2007; 56: 1265-1274.

Dutt V, Greenleaf JF. Adaptive speckle reduction filter for log-compressed B-Scan images. IEEE Transactions On Medical Imaging 1996; 15: 802-813.

Fan L, Santiago P, Jiang H, Herrington DM. Ultrasound measurement of brachial flow-mediated vasodilator response. IEEE Transactions On Medical Imaging 2000; 19: 621-631.

Fan L, Santiago P, Riley W, Herrington D. An adaptive template-matching method and its application to the boundary detection of brachial artery ultrasound scans. Ultrasound in Medicine \& Biology 2001; 27: $399-408$.

Hoeks APG, Willekes C, Boutouyrie P, Brands PJ, Willigers JM, Reneman RS. Automated detection of local artery wall thickness based on m-line signal processing. Ultrasound in Medicine \& Biology 1997; 23: 10171023.

Kurtz A. Mirror-image artifact mimicking a carotid artery dissection. Journal of Diagnostic Medical Sonograohy 2008; 24: 26-29.

Meinders JM, Brands PJ, Willigers JM, Kornet L, Hoeks APG. Assessment of the spatial homogeneity of artery dimension parameters with high frame rate 2-D B-mode. Ultrasound in Medicine \& Biology 2001a; 27: 785-794.

Meinders JM, Kornet L, Brands PJ, Hoeks APG. Assessment of local pulse wave velocity in arteries using 2D distension waveforms. Ultrasonic Imaging 2001b; 23: 199-215.

Meinders JM, Hoeks APG. Simultaneous assessment of diameter and pressure waveforms in the carotid artery. Ultrasound in Medicine \& Biology 2004; 30: 147-154.

Paini A, Boutouyrie P, Calvet D, Zidi M, Agbiti-Rosei E, Laurent S. Multiaxial mechanical characteristics of carotid plaque. Analysis by multiarray echotracking system. Stroke 2007; 38: 117-123.

Pannier BM, Avolio AP, Hoeks APG, Mancia G, Takazawa K. Methods and devices for measuring arterial compliance in humans. American Journal of Hypertension 2002; 15: 743-753.

Reneman RS, Meinders JM, Hoeks APG. Non-invasive ultrasound in arterial wall dynamics in humans: what we have learned and what remains to be solved. European Heart Journal 2005; 26: 960-966.

Reneman RS, Arts T, Hoeks APG. Wall shear stress - an important determinant of endothelial cell function and structure - in the arterial system in vivo. Journal of Vascular Research 2006; 43: 251-269. 
Schmidt-Trucksass A, Cheng D, Sandcrock M, Sculte-Monting J, Rauramaa R, Huonker M, Burkhardt H. Computerized analyzing system using the active contour in ultrasound measurement of carotid artery intima-media thickness. Clinical Physiology 2001; 21: 561-569.

Sonka M, Liang W, Lauer RM. Automated analysis of brachial ultrasound image sequences: early detection of cardiovascular disease via surrogates of endothelial function. IEEE Transactions On Medical Imaging 2002; 21: 1271-1279.

Tao Z, Jaffe CC, Tagare HD, Tunnelling descent: a new algorithm for active contour segmentation of ultrasound images. In: Information Processing in Medical Imaging, Springer Berlin / Heidelberg, 2003.

Tao Z, Tagare HD, Beaty JD. Evaluation of four probability distribution models for speckle in clinical cardiac ultrasound images. IEEE Transactions On Medical Imaging 2006; 25: 1483-1491.

Touboul P-J, Hennerici MG, Meairs S, Adams H, Amarenco P, Bornstein N, Csiba L, Desvarieux M, Ebrahim S, Fatar M, Hernandez Hernandez R, Jaff M, Kowantor S, Prati P, Rundek T, Sitzer M, Schminke U, Cardif JC, Taylor A, Vicaut E, Woo KS, Zannad F, Zureik M. Mannheim carotid intima-media thickness consensus (2004-2006). Cerebrovascular Diseases 2007; 23: 75-80.

Udupa JK, LeBlanc VR, Zhuge Z, Imieliska C, Schmidt H, Currie LM, Hirsch BE, Woodburn J. A framework for evaluating image segmentation algorithms. Computerized Medical Imaging And Graphics 2006; 30: 75-87.

Van Bortel L, Vanmolkot FH, Van der Heijden-Spek JJ, Bergu M, Staessen JA, Hoeks APG. Does B-mode common carotid artery intima-media thickness differ from $\mathrm{m}$-mode? Ultrasound in Medicine \& Biology 2001; 27: 1333-1336.

Wagner RF, Smith SW, Sandrick JM, Lopez H. Statistics of speckle in ultrasound B-scans. IEEE Transactions On Sonics And Ultrasonics 1983; 30: 156-163.

Wu A, Xu D, Yang X, Zheng J, Generic solution for image object recognition based on vision cognition theory. In: Fuzzy Systems and Knowledge Discovery, Springer Berlin / Heideberg, 2005.

Zhang X, McKay CR, Sonka M. Tissue characterization in intravascular ultrasound images. IEEE Transactions On Medical Imaging 1998; 17: 889-899. 


\section{CHAPTER 5}

\section{Automatic estimation of common}

carotid artery diameter, orientation and curvature

The content of this chapter is based on the publication: Rossi AC, Brands PJ, Hoeks APG. Fully automatic assessment of carotid artery curvature and diameter with noninvasive ultrasound. 2008. IFMBE Proceedings, 4th European Conference of the International Federation for Medical and Biological Engineering, (Antwerp, Belgium, 23-27 November 2008); 22: 588-591. 


\section{1-Abstract}

An algorithm for performing fully automatic morphological analysis of the common carotid artery (CCA) in longitudinal ultrasound (US) B-mode scans is introduced. Arterial position, curvature and diameter are automatically estimated offline without human supervision. The CCA is automatically recognized by applying a method based on decimation, template matching and clustering to the first pair of US frames. This allows automatic placement of seed points in the CCA lumen. Next a sustain-attack filter (SAF) is applied in order to get raw estimates for CCA wall positions, used to deduct the CCA centerline in a region of interest (ROI). Arterial orientation and curvature are estimated by fitting a second order polynomial through the centerline. Subsequently the lines of sight in the ROI are vertically re-aligned based on the centerline so that the arterial walls shift to an approximately horizontal position, allowing subsequent lateral smoothing with horizontal kernels. This directional pre-processing attenuates speckle modulation without blurring perpendicularly to CCA walls. The SAF is then re-applied, yielding smooth delineation of both near and far wall. CCA diameter, orientation and curvature radius are calculated within the ROI. Automatic tracking of CCA movements is performed frame-by-frame. The method is tested on 30 US recordings (duration 6 seconds each) from 10 healthy volunteers. The algorithm successfully detects, without any user interference, CCA position, curvature and diameter for every subject considered. Comparison of results with and without curvature-compensated lateral smoothing shows significant decreases in the standard deviation of wall position estimates when the proposed pre-processing is applied. Correcting for local orientation at each line of sight yields the real CCA diameter, eliminating the error due to a non-perpendicular US beam orientation.

\section{2 - Introduction}

Many morphological and dynamic properties of the common carotid artery (CCA), such as diameter, distension and intima-media thickness, can be estimated noninvasively by means of transcutaneous B-mode ultrasound (US) (Reneman 2005). Since the CCA often appears slightly tilted and/or curved in US images due to its non-parallel orientation with respect to the transducer surface, information on local orientation and curvature is also needed. Usually CCA diameter and intima-media thickness estimation are measured along the US beam (Graf 1999), assuming it to be exactly perpendicular to the artery. Knowing the local orientation would give the possibility to abandon this assumption. Also Doppler analysis (Hoeks 1981) would benefit from such angle knowledge. Moreover, current vascular US segmentation 
methods are semi-automatic, since they require manual recognition of the CCA prior to the analysis.

In this chapter an algorithm performing fully automatic assessment of curvature and diameter in B-mode scans of the CCA is presented. The method automatically crops a region of interest ( $\mathrm{ROI}$ ) around the CCA, without any user interference, and then proceeds to estimate diameter and curvature on a frame-by-frame basis. The local curvature of the vessel centerline is exploited to perform a "directional smoothing" of the arterial walls, in order to make edge detection less sensitive to speckle without losing resolution along the beam (in depth).

The artery is properly recognized, and its adventitial walls and centerline properly delineated, in each frame of 30 US recordings (duration 6 seconds each) from 10 healthy subjects. Diameter, orientation and curvature are estimated both along the ROI width and in time. For each subject precision consistently improves by applying directional smoothing as a pre-processing step on the images. Angle information is used to correct the diameter values measured along the US beams, yielding the real CCA diameter.

\section{3 - Materials and methods}

\subsection{1 - Ultrasound acquisitions}

Common carotid arteries of 10 healthy volunteers (age ranging from 15 to 40, 6 males and 4 females) were imaged with ultrasound. The study was approved by the joint ethical committee of Maastricht University and Academic Hospital Maastricht. All subjects gave informed consent prior to enrolment. B-mode scans were performed using ART.LAB (ESAOTE EUROPE BV, Maastricht, The Netherlands), a software-based radio frequency US acquisition system (Brands 1999). For each subject three subsequent acquisitions were recorded, each one covering a time interval of 6 seconds. Each acquisition resulted in storage of both digitized RF echo signals and image grey levels for each US frame (frame rate $30 \mathrm{fps}, 180$ frames per acquisition). A frame consists of 128 lines of sight, covering $40 \mathrm{~mm}$ in width and $35 \mathrm{~mm}$ in depth. The center frequency of the linear array used was $7.5 \mathrm{MHz}$, and its pitch was 315 $\mu \mathrm{m}$. RF echo signals were sampled at a frequency of $33 \mathrm{MHz}$ (sample distance 23 $\mu \mathrm{m}$ ) with a dynamic range of $72 \mathrm{~dB}$. B-mode image columns were also captured, after logarithmic compression and thresholding, at $16 \mathrm{MHz}$ sampling rate with 8 bits 
encoding. All the processing was then performed offline, using Matlab (MathWorks, Natik, MA).

\subsection{2 - Automatic CCA recognition}

The first two B-mode frames of a recorded sequence are sufficient to automatically recognize the CCA without any user interaction. The authors previously introduced the algorithm for automatic CCA recognition (Rossi 2008) in Chapter 4. A subset of the echo amplitude signals in each frame is decimated according to the US system resolution, and is then subjected to parametrical template matching exploiting the typical diameter range of the CCA in adults. Results of template matching in the two frames are gathered and clustered to reject outliers. A linear fit is performed over the automatically placed seed points (Fig. 5.1) to calculate the average lumen center position. A $20 \mathrm{~mm}$ wide and $16 \mathrm{~mm}$ deep ROI is subsequently centered on the artery. From frame nr. 2 onwards the processing for orientation, curvature and diameter estimation involves echo amplitudes derived from the stored RF signals, thereby avoiding the influence of the logarithmic compression performed by the US scanner on B-mode images. Automatic tracking of CCA movements in time is achieved by displacing the ROI according to the results of artery wall delineation for each frame.

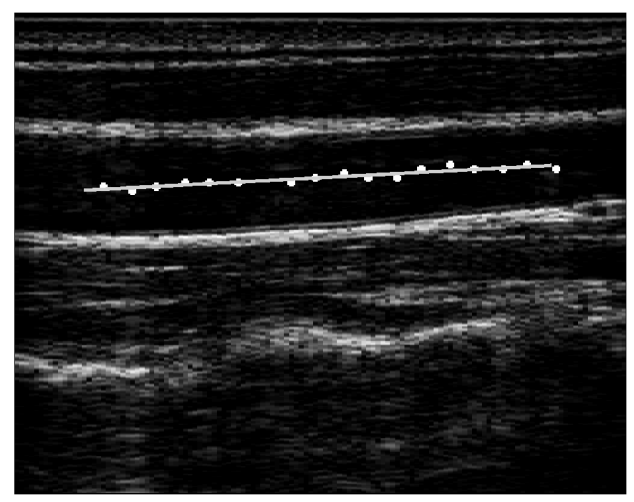

Fig. 5.1 - The algorithm automatically identifies the CCA. Automatically placed seed points (white circles) are subsequently concatenated by a linear fit (grey line).

\subsection{3 - Sustain-attack filter and centerline}

Envelopes of the 64 RF signal segments in the ROI are computed by means of timedomain Hilbert transform (Brands 1997). The edge detector used to locate the adventitial walls is the so called sustain-attack filter (SAF) (Meinders 2001), applied along depth both in forward and backward direction. The SAF, described in Chapter 
3 , is based on exponentially decaying threshold functions, always related to the last dominant echo while following gradual changes in echo level along the line of sight. Given the lumen position, the SAF directly supplies a fist guess for the location of the CCA media-adventitia interfaces for each envelope signal in the ROI. Second order polynomial fits are performed on both anterior (near) and posterior (far) wall tracings. To reject outliers, the regions in which the deviation between the SAF tracing and the fit are higher than the average deviation are smoothed by setting them to the polynomial fit values. The CCA centerline is obtained with a second order polynomial fit through the middle points between adventitial positions for each line of sight (Fig. 5.2a). The smooth vessel centerline thus obtained for each frame is used to estimate local orientation and curvature of the vessel.
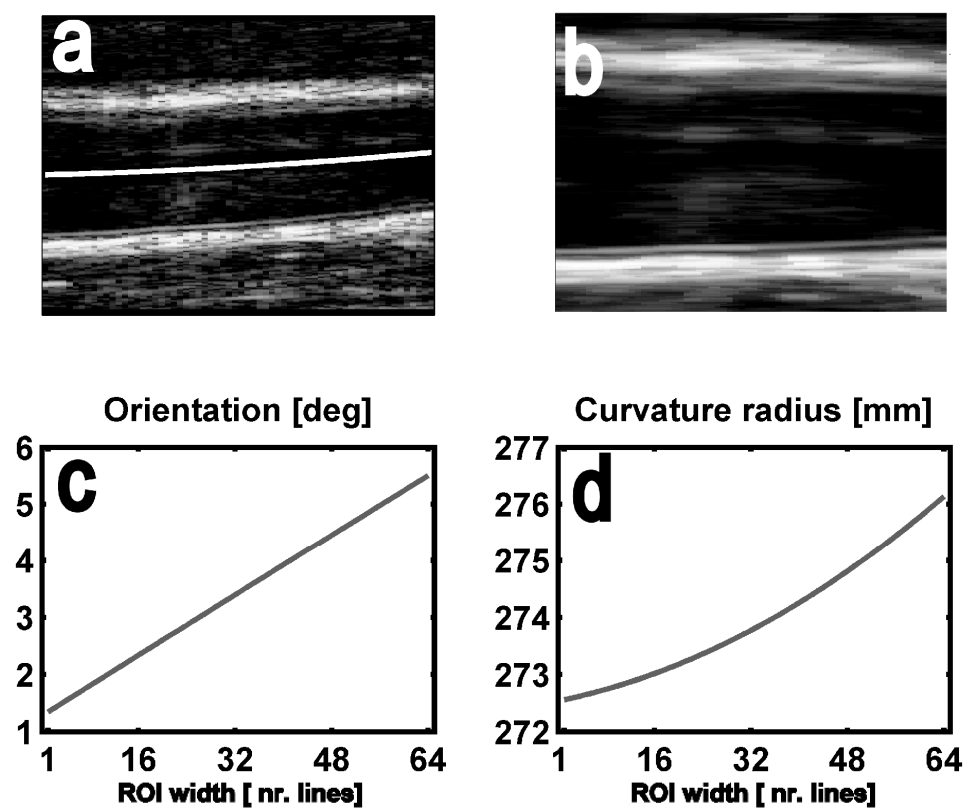

Fig. 5.2 - a) ROI with overlaid CCA centerline; b) effect of realignment and directional smoothing; c) orientation and d) curvature radius in the ROI.

Denoting ROI width with $w$ and the centerline depth as $F c(w)$, the local orientation (Fig. 5.2c) is

$$
A(w)=\arctan [d F c(w) / d w]
$$

while the local curvature (Fig. 5.2d) is calculated as 


$$
K(w)=\frac{d^{2} F c(w) / d w^{2}}{\left[1+[d F c(w) / d w]^{2}\right]^{3 / 2}}
$$

The radius of curvature is computed as $\rho(w)=1 /|K(w)|$.

\subsection{4 - Directional smoothing}

All lines of sight in the ROI are re-aligned, based on the centerline, to shift arterial walls to an approximately horizontal orientation. At this point, a lateral median filtering is applied with a kernel 1 sample deep and 9 samples (i.e. $2.5 \mathrm{~mm}$ ) wide. This will despeckle the US data without blurring perpendicularly to the arterial walls (Fig. 5.2b), exploiting echo amplitude consistency in the direction parallel to the CCA centerline.

\subsection{5 - CCA diameter estimation}

The SAF is re-applied on each envelope signal in the directionally filtered ROI, yielding a smooth delineation of the adventitial borders of the CCA for every frame (Fig. 5.5). In order to compute the average CCA diameter in the ROI, the local diameters measured along the ultrasonic beam are corrected for the observed local orientation of the artery, and then averaged. The procedure provides the real diameter of the CCA, which will be generally smaller than the observed diameter. CCA diameter waveform, average CCA orientation and average CCA curvature radius are obtained as function of time (Fig. 5.3).

\section{4 - Results}

The proposed method performed correct automatic CCA recognition, centerline tracking, and estimation of arterial diameter, orientation and curvature for each one of the 30 US acquisitions analyzed, without the need of human supervision during offline processing. Standard deviations of both adventitial wall tracings within frames were evaluated with and without directional smoothing, and then averaged over the 3 repeated acquisitions (Fig. 5.4). Differences in diameter values were evaluated between the cases with and without orientation compensation. 

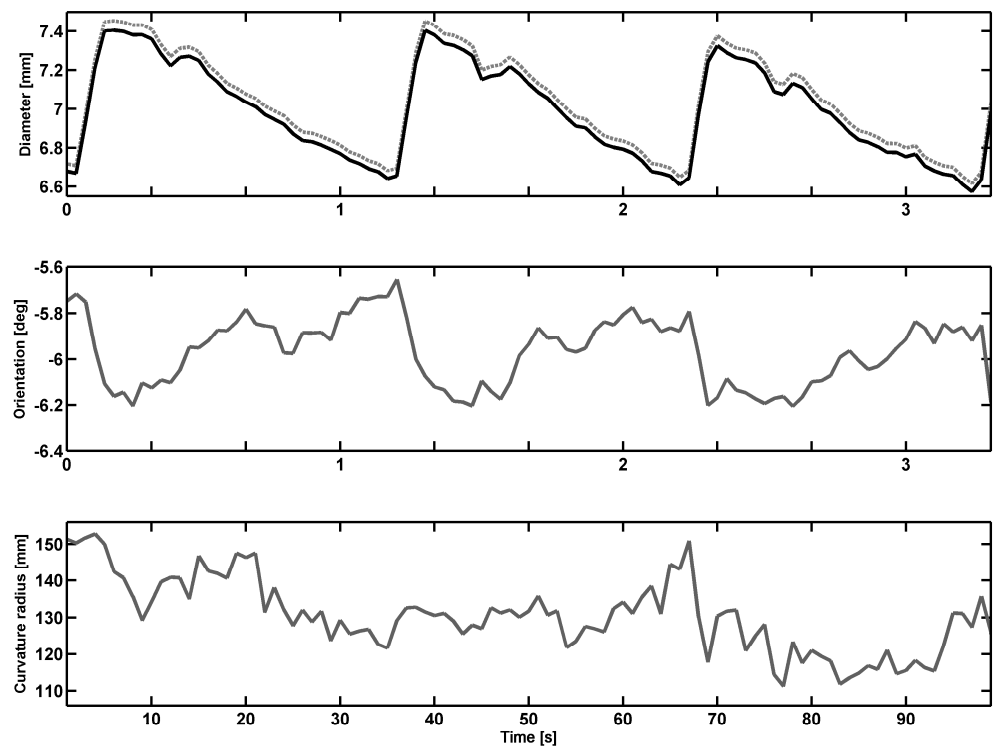

Fig. 5.3 - Top: example of CCA diameter waveform with (black solid line) and without (grey dashed line) orientation compensation; center: average CCA orientation; bottom: average CCA curvature radius.

The proposed method performed correct automatic CCA recognition, centerline tracking, and estimation of arterial diameter, orientation and curvature for each one of the 30 US acquisitions analyzed, without the need of human supervision during offline processing. The standard deviations of both adventitial wall tracings within frames were evaluated with and without directional smoothing, and then averaged over the 3 repeated acquisitions (Fig. 5.4). Differences in diameter values were evaluated between the cases with and without orientation compensation. The obtained wall and centerline tracings (Fig. 5.5) were remapped for a number of frames, in order to visually inspect the results of the segmentation.

\section{5 - Discussion}

The precision of adventitia-adventitia CCA diameter assessment improves significantly by exploiting the vessel centerline profile in order to directionally despeckle US envelopes. Fig. 5.4 shows that this happens for both CCA walls, with up to $70 \mu \mathrm{m}$ decrease in the standard deviation of wall position estimates. The variability of the 
near wall position is always slightly higher than that of the far wall because for the anterior wall the SAF algorithm considers the trailing echo edges.
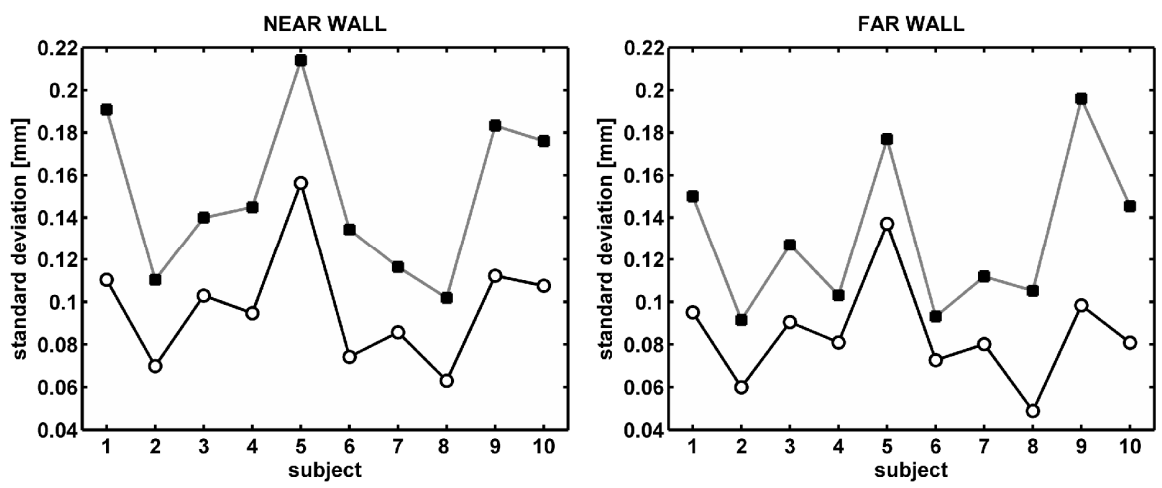

Fig. 5.4 - Standard deviations of wall position estimation for the CCA near (left) and far wall (right). Precision is consistently improved by using directional smoothing (white circles).

Fig. 5.5 illustrates the effect of directional smoothing on wall delineation. CCA adventitial boundaries are more realistic and smooth when the proposed despeckling procedure is applied.
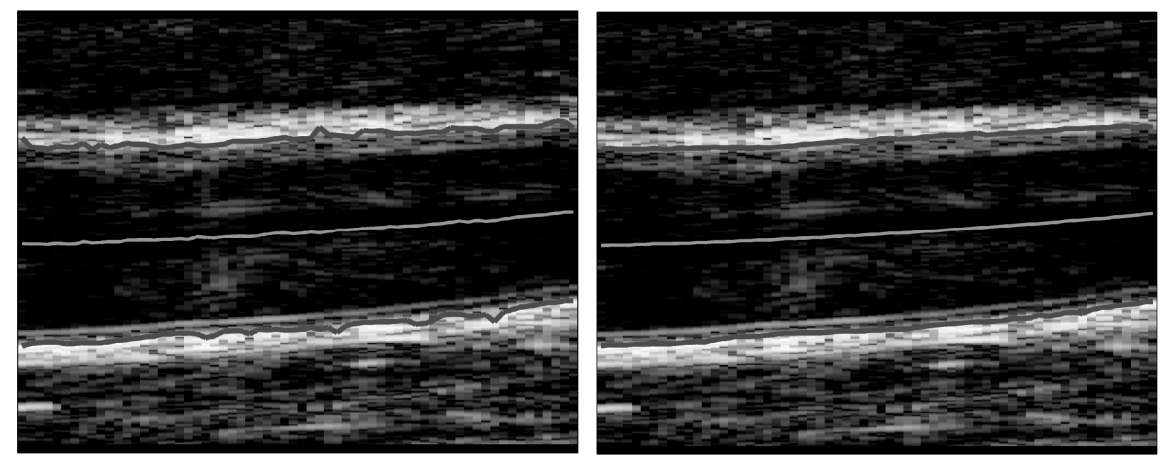

Fig. 5.5 (color version on page 150) - Left: no directional smoothing; right: results obtained with directional smoothing, remapped on the original ROI. The contours are obtained with the sustain-attack filter. The processing occurs on linear envelopes, but here images are shown with logarithmic compression for visual convenience.

Since the processing occurred on RF signals having an intersample distance of 23 $\mu \mathrm{m}$, this guarantees a higher definition in wall delineation with respect to direct analysis of B-mode images. Sampling a $16 \mathrm{~mm}$ deep ROI of a digitized B-mode frame with 500 pixels in depth would still yield an intersample distance of $32 \mu \mathrm{m}$, which is a factor 1.4 larger. A small intersample distance would be advantageous also in 
cases in which smoothing in both directions would be necessary, since it guarantees that speckle noise fluctuations cover more than two samples.

The knowledge of CCA orientation and curvature within the ROI allows compensation for vessel angulation when measuring the diameter. Fig. 5.3 (top) shows the difference between compensated and uncompensated diameter waveforms for a subject having an average CCA angulation of about -6 degrees. Average differences in diameter with and without angle correction ranged between 10 to $50 \mu \mathrm{m}$ across subjects. Since the study evaluated only US acquisitions from healthy volunteers, the imaged CCA segments were rarely tilted more than 10 degrees with respect to the probe surface. However, when dealing with patients, the CCA can be much more irregularly oriented due to pathologies, aging, and plaques, and this can introduce larger differences between the diameter measured locally along the US beam and the real one.

The average CCA orientation in the ROI evolves in phase with the diameter waveform (Fig. 5.3, center). This indicates that the pulse pressure induces a slight periodical tilting of the artery along with its dilation. Curvature radius fluctuations are instead more random (Fig. 5.3, bottom). Assessing the time evolution of CCA orientation and curvature together with CCA diameter gives the possibility to ensure that the US B-mode scan plane is properly positioned on the vessel, for example by checking that the fluctuations in angle and curvature radius are not exceeding some predefined limits. If implemented in real-time, this would help sonographers to maintain a proper scan plane throughout the measurement.

\section{6-Conclusion}

In this chapter we introduce a method to perform fully automatic assessment of CCA adventitia-adventitia diameter, orientation and curvature without user interaction (i.e. without manually placed seed points or manual ROI cropping prior to delineation). The method is robust and reliable, also due to directional despeckling taking advantage of the CCA assessed centerline for each US frame, which improves the overall precision. Further developments will extend the same technique to CCA intima-media thickness estimation. The algorithm could also be applied to other vascular districts of interest in US imaging, such as brachial and femoral arteries. In Doppler systems the line-by-line estimation of arterial orientation would facilitate proper conversions of measured Doppler frequencies to velocities corrected for the actual angle between local blood flow and ultrasonic beam direction. In the present implementation such correction is applied to the measured CCA diameter, thus 
providing measurements perpendicularly to the CCA centerline regardless of its orientation with respect to the probe.

\section{References}

Brands PJ, Hoeks APG, Ledoux LAF, Reneman RS. A radio frequency domain complex cross-correlation model to estimate blood flow velocity and tissue motion by means of ultrasound. Ultrasound in Medicine \& Biology 1997; 23: 911-920.

Brands PJ, Hoeks APG, Willigers J, Willekes C, Reneman RS. An integrated system for the non-invasive assessment of vessel wall and hemodynamic properties of large arteries by means of ultrasound. European Journal of Ultrasound 1999; 9: 257-266.

Graf S, Gariepy J, Massoneau M, Armentano RJ, Mansour S, Barra JG, Simon A, Levenson J. Experimental and clinical validation of arterial diameter waveform and intimal media thickness obtained from B-mode ultrasound image processing. Ultrasound in Medicine \& Biology 1999; 25: 1353-1363.

Hoeks APG, Reneman RS. A multigate Doppler system with serial data processing. IEEE Transactions On Sonics And Ultrasonics 1981; SU-28: 242-247.

Meinders JM, Brands PJ, Willigers JM, Kornet L, Hoeks APG. Assessment of the spatial homogeneity of artery dimension parameters with high frame rate 2-D B-mode. Ultrasound in Medicine \& Biology 2001; 27: 785-794.

Reneman RS, Meinders JM, Hoeks APG. Non-invasive ultrasound in arterial wall dynamics in humans: what we have learned and what remains to be solved. European Heart Journal 2005; 26: 960-966.

Rossi AC, Brands PJ, Hoeks APG. Automatic recognition of the common carotid artery in longitudinal ultrasound B-mode scans. Medical Image Analysis 2008; 12: 653-665. 


\section{CHAPTER 6}

\section{Localization of common carotid artery intimal and adventitial layers by means of ultrasound}

The content of this chapter is based on the original paper, submitted for publication: Rossi AC, Brands PJ, Hoeks APG. Automatic localization of intimal and adventitial carotid artery layers with non-invasive ultrasound: a novel algorithm providing scan quality control. 2009. 


\section{1-Abstract}

Transcutaneous ultrasonic measurements of common carotid artery (CCA) diameter and intima-media thickness (IMT) give insight on arterial dynamics and anatomy, both correlating well with atherosclerosis and cardiovascular risk. We propose a novel automatic algorithm to estimate CCA diameter and IMT in ultrasound (US) images, based on separate analysis of anterior and posterior CCA walls and able to distinguish internal (intima-intima) and external (adventitia-adventitia) diameter. The method combines off-line signal- and image-processing techniques to accommodate echo images composed directly from RF data, not involving digital videograbbing. Segmentation consists of automatic CCA recognition, adventitial delineation by means of sustain-attack filter, and intimal delineation based on Multiscale Anisotropic Barycenter (MAB). An automatic measure of the quality of the US beam incidence for each wall is superimposed on the CCA contour overlays for visual feedback. Validation is carried out on 36 US CCA acquisitions from 12 healthy volunteers, as well as on synthetic US images. Results indicate good accuracy and precision, with Intra-recording beat-to-beat variations on average lower than $50 \mu \mathrm{m}$ for external diameter and IMT, and lower than $100 \mu \mathrm{m}$ for internal diameter. The beam incidence control significantly increases the repeatability of IMT estimates, and is intended to actively motivate sonographers to maintain a proper scan plane throughout the acquisition to minimize the incidence of confounding factors in noninvasive CCA ultrasonic examinations. The method is clinically viable, providing robust estimates of CCA diameter and IMT waveforms, even at a low B-mode update rate of 30 frames per second.

\section{2 - Introduction}

Non-invasive estimates of common carotid artery (CCA) anatomical features by means of ultrasound (US), including diameter and intima-media thickness (IMT), are gaining importance for prevention and treatment of cardiovascular diseases, assessment of cardiovascular risk, and estimation of arterial mechanical properties (Riley 1997; De Groot 2004; Reneman 2005; Laurent 2006; Mattace-Raso 2006; Baldassarre 2008; Hoeks 2008). Many different image processing methods are nowadays available for assessing CCA diameter and IMT on 2-dimensional ultrasonic images, usually based on the grey level gradient or on active contours (Selzer 1994; Schmidt-Trucksass 2001; Stein 2005). Recently, an image processing method has been presented for image edge detection, based on the "first absolute central moment" as alternative to gradient-based analysis (Demi 2000). It has also been extended for the automatic analysis of arterial B-mode US scans (Gemignani 2007; Faita 2008). 
In this chapter we introduce a new algorithm, based on the central moment mathematical operator (Demi 2000), for the automatic estimation of CCA internal and external diameter, together with IMT of both arterial walls. The algorithm works in conjunction with an automated CCA recognition protocol (Rossi 2008b) and with the so called "sustain-attack filter" (Hoeks 1995; Meinders 2001) used for adventitial wall positioning. The intima detection on both walls is based on a multiscale approach computing iteratively the barycenter (centre of gravity) of the echo level variation within circular image domains of varying size, adapted for anisotropic sampling. The latter property allows processing of US envelopes, which are not yet scan converted but derived directly from the set of underlying radio frequency (RF) signals in a frame. The proposed intimal detection approach, named Multiscale Anisotropic Barycenter (MAB), considers images in their original linear grey level scale without logarithmic compression, since non-invasive vascular US measurements are affected by intimal-medial reflectivity (Ellis 2007) and dynamic range settings (Potter 2008; Rossi 2009). Dedicated post-processing integrates contour estimation with outlier detection and unbiasing to compensate for differences in edge detection for the media-adventitia and lumen-intima interfaces.

Non-invasive ultrasonic arterial measurements in the longitudinal CCA plane are affected by the angle of incidence between the ultrasonic beam and the artery. A proper and consistent insonation plane is crucial both for diameter (Stadler 1996; Dineley 2007) and IMT assessment (Bruschi 1997). The currently available 3D ultrasound systems have specific applications in the complementary fields of wallvolume or plaque-volume estimation (Spence 2006; Egger 2008; Mallett 2008), but IMT estimation is still better performed with 2-dimensional ultrasound. Since the method is aimed at the estimation of CCA diameter and IMT and not at the analysis of atherosclerotic plaques, longitudinal 2-dimensional US is the optimal imaging choice. We integrated in the algorithm an automatic procedure that evaluates the adequacy of the insonation plane for each US frame, utilizing the outcome of the automatic CCA segmentation process. This procedure also allows quantification of the effects of "out of plane" US frames on the arterial parameters estimated.

We evaluated the algorithm on repeated longitudinal CCA US acquisitions from 12 young healthy volunteers to establish the suitability of the proposed method for the detection of external (adventitia-adventitia) CCA diameter estimation, internal (intima-intima) CCA diameter estimation, and for IMT assessment of both CCA walls. We focused on the CCA since it has more consistent echogenic properties than the internal carotid artery or the carotid bulb (Touboul 2007). An accuracy analysis was also carried out, using synthetic US images. Near wall and far wall IMT were analyzed separately and compared in order to investigate whether observed differ- 
ences originate from different echo-generation characteristics; the IMT scanning protocol is one of the potential confounding factors when comparing populationbased studies (Lorenz 2007). The aim of our work is the development of an automatic CCA segmentation method that exploits the information contained in the ultrasonic radio frequency (RF) echo signals to provide precise and repeatable delineation of the artery walls, to be used for internal and external CCA diameter estimation. We also want to show that the variability of the IMT estimates can decrease if the US scan plane properties are taken into account.

\section{3 - Materials and methods}

\subsection{1 - Acquisitions}

The left common carotid artery of 12 healthy subjects (age ranging from 15 to 40 years, 8 males and 4 females) were scanned in B-mode. The study was approved by the joint ethical committee of Maastricht University and Academic Hospital Maastricht. All subjects gave informed consent prior to enrolment.

A software-based radio frequency ultrasound (US) acquisition system, ART.LAB (ESAOTE EUROPE BV, Maastricht, The Netherlands), was used to perform B-mode scans (Brands 1999). Arteries were scanned in longitudinal sections with perpendicular incidence, and the transmit focus was set at a depth of $2 \mathrm{~cm}$, which corresponded to the whereabouts of the CCA lumen. Three subsequent acquisitions were performed for each subject, resulting in a total of 36 recordings, each one covering a time interval of 6 seconds and therefore about 4-6 heart beats, depending on the heart rate of the subject. Prior to the acquisitions in supine position, each subject was given a rest period of 10 minutes. The subjects were asked to hold their breath for the brief duration of each measurement to eliminate possible variability in CCA diameter and IMT due to the respiratory cycle. Particular attention was paid to the gain settings in the ART.LAB system to avoid signal saturation.

Each acquisition resulted in storage of digitized RF echo signals. The stored file contains 180 matrixes (frame rate $30 \mathrm{fps}$, acquisition time $6 \mathrm{~s}$ ), each composed of 128 RF signals, covering $40 \mathrm{~mm}$ in width and $35 \mathrm{~mm}$ in depth. The center frequency of the linear array was $7.5 \mathrm{MHz}$, while the line pitch was $315 \mu \mathrm{m}$. The RF signals were sampled at a frequency of $33.3 \mathrm{MHz}$ (inter-sample distance $23.1 \mu \mathrm{m}$ ) with a dynamic range of $72 \mathrm{~dB}$ (12 bits stored as the most significant bits of a 16 bits word). The acquired RF signals come from a stage in the signal processing chain of the US scanner where the signals have still a linear amplitude range. The absence of logarithmic compression avoids undesirable effects on CCA US assessments (Rossi 2009). More- 
over, the high RF sampling frequency in depth guarantees a higher definition in wall delineation with respect to direct analysis of B-mode images with inter-sample distances after scan conversion usually in the order of $70 \mu \mathrm{m}$. For the purpose of automatic CCA segmentation, the RF matrixes were subjected to off-line processing using MATLAB (MathWorks, Natik, MA).

\subsection{2 - Automatic CCA recognition and RF envelopes}
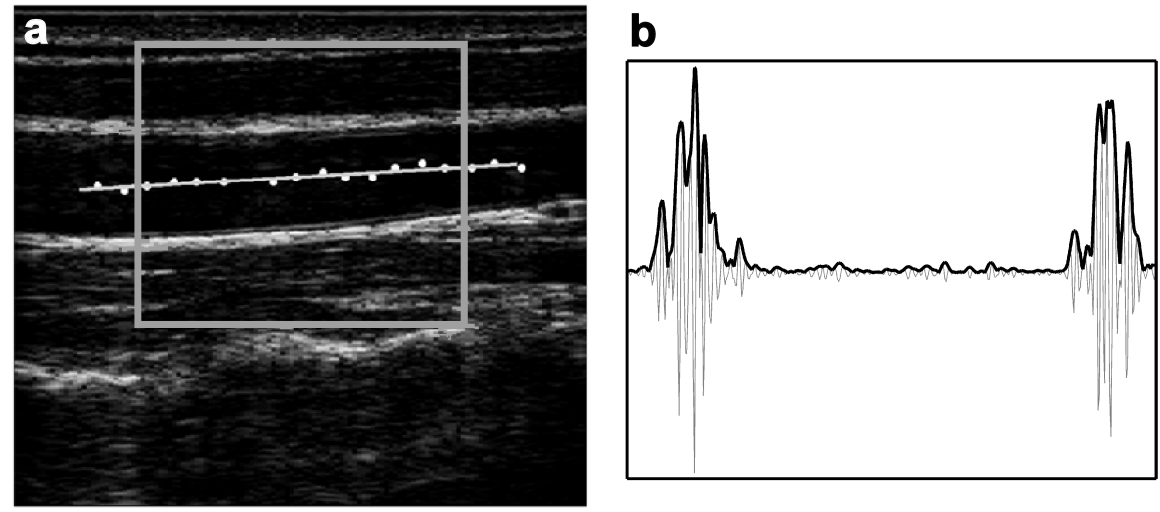

Fig. 6.1 - a) The automatically cropped ROI (grey box) provided by the automatic CCA recognition algorithm (white dots) with a linear fit for the identified lumen center line; b) RF signal (grey line), covering both near and far wall of the CCA, with superimposed its envelope (black line).

Within each acquired RF frame a region of interest (ROI) is automatically cropped (Fig. 6.1a), covering a depth of $20 \mathrm{~mm}$ (i.e. 866 samples) and a width of $23 \mathrm{~mm}$ (i.e. 74 signals). In the horizontal direction the ROI is centered in the middle of the frame, whereas its position in depth is automatically adjusted to the CCA lumen. The automatic recognition of the CCA is achieved by means of a previously developed algorithm (Rossi 2008b), based on decimation, template matching and clustering, applied to the first couple of frames in the stream. Over subsequent frames the ROI position is automatically updated according to the vertical displacement of the CCA as outcome of the vessel wall contour delineation, described further in the chapter. The DC component is removed from each vertical RF signal segment in the ROI prior to time-domain Hilbert transformation to a complex analytic signal for envelope computation (Fig. 6.1b). The discrete Hilbert impulse response is set to 7 non-zero samples on both positive and negative side, minimizing amplitude and phase errors (Brands 1997). The instantaneous envelope of the RF signal is the absolute value of the complex analytic signal. Even though the envelopes considered are not logarithmically compressed, all images showing US frames in this chapter incor- 
porate logarithmic compression for visual convenience (whereas all graphical overlays refer to the processing of linear envelopes).

\subsection{3 - Adventitial wall delineation}

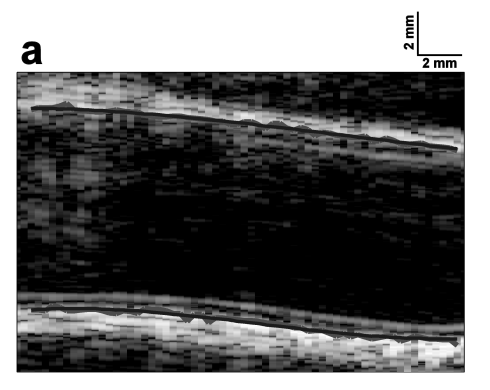

b

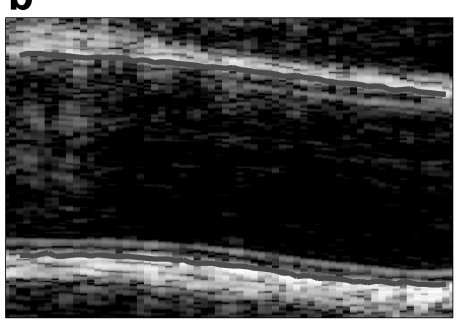

C

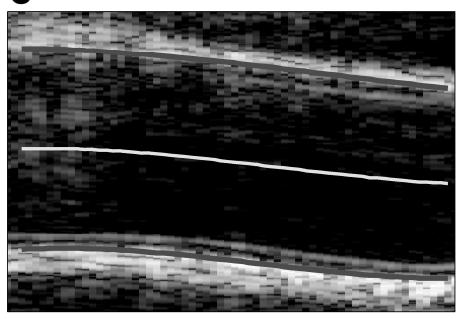

Fig. 6.2 (color version on page 151) - Delineation of adventitial contours and centerline. a) The outcome of the sustain-attack filter is fitted with a third-order polynomial; b) outlier rejection is performed; c) an additional third-order fit is performed in order to smoothly follow the arterial curvature; the centerline follows from the anterior and posterior wall positions.

For notational convenience, we will from now on refer to the CCA walls as "near wall" and "far wall". The detection of the media-to-adventitia interface on each wall of the CCA is based on a sustain-attack filter (Hoeks 1995; Meinders 2001; Rossi 2009). Such filter generates exponentially decaying threshold functions acting separately on each RF envelope in forward (near wall) and backward (far wall) direction. This allows the method to locate media-adventitial interfaces independent of local echo amplitude and gain settings. The mathematical details regarding the sustainattack filter can be found in Chapter 3 . The detected wall positions for each wall are 
fitted with a third order polynomial across the ROI (Fig. 6.2a). Outliers with a deviation from the polynomial fit of more than the root mean square error are substituted with values of the polynomial fit (Fig. 6.2b). The updated set of adventitial wall positions is again smoothed by a third order polynomial fit. The arterial centerline is obtained by averaging near and far wall positions for each signal (Fig. 6.2c). The centerline serves as reference to derive orientation and curvature of the CCA (Rossi 2008a).

\subsection{4 - Intimal wall delineation: barycenter of the echo level deviation}

The proposed detection method for the lumen-intima interface is an extension of the "first order absolute central moment" (Demi 2001; Gemignani 2004). The method was originally developed for the detection of brachial artery diameter in digitally grabbed B-mode US images (square pixels) to quantify flow-mediated dilation (Gemignani 2007). Recently the principle of first order central moment was also suggested for carotid intima-media thickness detection (Faita 2008). The algorithm is based on the centre of gravity (barycenter) of grey level deviations (relative to a local mean) within a circular evaluation domain containing a grey level discontinuity. In the case of vascular ultrasound, the discontinuity of interest is the one between the vessel lumen and the intima. The barycenter of the echo level deviation is based on the spatially weighted absolute value of echo variation, defined as the difference between a pixel value and a local average level. Spatial weighting in the circular evaluation domain and averaging in a smaller concentric smoothing domain are achieved with Gaussian kernels. The standard deviation of each Gaussian kernel is $1 / 3$ of the radius of the respective circular domain. It is demonstrated (Demi 2001) that when the evaluation domain contains a grey level discontinuity, the located barycenter is always closer to the discontinuity than the domain's geometrical centre regardless of position of the discontinuity within the domain. This feature facilitates an iterative approach for image edge detection, starting from a seed point in the artery lumen that will get stepwise closer to the edge. The process is independent of the spatial orientation of the boundary because of the circular shape of the domain. A detailed mathematical description can be found in Appendix 6A.

In the original implementation (Gemignani 2007) the procedure had to be manually initialized with an approximated contour close to the vessel boundary, whereas the number of iterations was empirically limited to 5 . Here we introduce a modified version for the iterative computation of the barycenter to detect the lumen-intima boundaries at both CCA walls in B-mode US frames. The modifications allow the algorithm to cope with ultrasound images that are not scan-converted, tying the procedure to the intrinsic anisotropic resolution of US systems rather than to image pixel sizes. The method is made rather insensitive to its initialization position within 
the lumen by adapting the size of the evaluation domain throughout the iterations. Moreover, the contour is allowed to assume curvilinear shapes, although plaques are excluded. We named the method "Multiscale Anisotropic Barycenter" (MAB) in relation to the above-mentioned properties.

\subsection{5 - Multiscale anisotropic barycenter (MAB)}

The RF and envelope signals have anisotropic sampling along depth and width. In the depth direction the RF sampling period equals $23.1 \mu \mathrm{m}$, while in the lateral direction beams are separated by $315 \mu \mathrm{m}$ (the pitch). This yields a width-to-depth sampling period ratio of 13.6. The generation of the concentric Gaussian kernels takes this anisotropic situation into account: in order for their domains to be isotropic in spatial coordinates, they have to be anisotropic in sample coordinates. Due to the coarse sampling in width, the domains are slightly ellipsoidal rather than circularly symmetrical. For all iterations, the smoothing domain is maintained at 716 $\mu \mathrm{m}$ (31 samples) in depth (about twice the axial resolution) and $945 \mu \mathrm{m}$ (3 samples) in width (focal lateral resolution). The domains' sizes are initially assigned along depth, and the corresponding values in width are computed according to the anisotropic sampling ratio. The geometrical centre of both concentric domains is iteratively displaced and results eventually in a contour point (fig. 6.3a).

The intima search is performed in every US frame independently for near and far CCA walls. Seed points are placed automatically $2 \mathrm{~mm}$ above (near wall search) and below (far wall search) the detected CCA centerline (Fig 6.3a). Intima detection is restricted to 61 central signals in the ROI, spanning a width of approximately 1.9 $\mathrm{mm}$. Each one of the 122 placed seed points evolves independently from the others according to the local iterative MAB procedure. At the first iteration, the radius of the evaluation domain is set equal to the distance between the seed point and the adventitial border as detected with the sustain-attack filter (Fig. 6.3a). At each iteration, only the depth coordinate of the local barycenter is taken into account to displace the contour point towards the intima. The choice of constraining movements along the depth direction is based on the empirical finding that otherwise the detected intimal contour points are likely to cluster on zones with high contrast. This is caused by the nature of ultrasound signals, exhibiting phase interference within resolution cells, i.e. speckle (Burckhardt 1978). In fact, the local contrast in US frames is not always related to the actual nature of the underlying tissue structures, especially when these are poorly echogenic and have an extension in depth in the range of the system resolution, as it is the case for the blood-intima interface in an artery. 


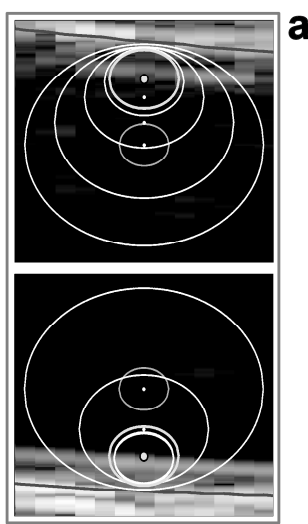

a

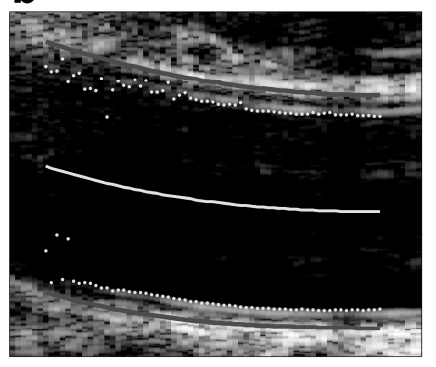

\section{C}

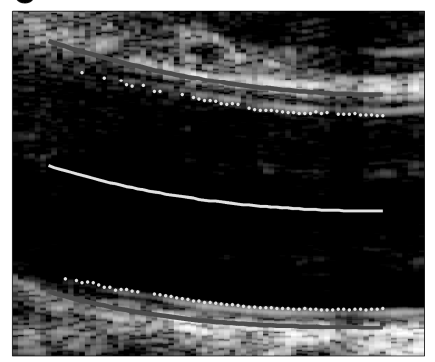

e
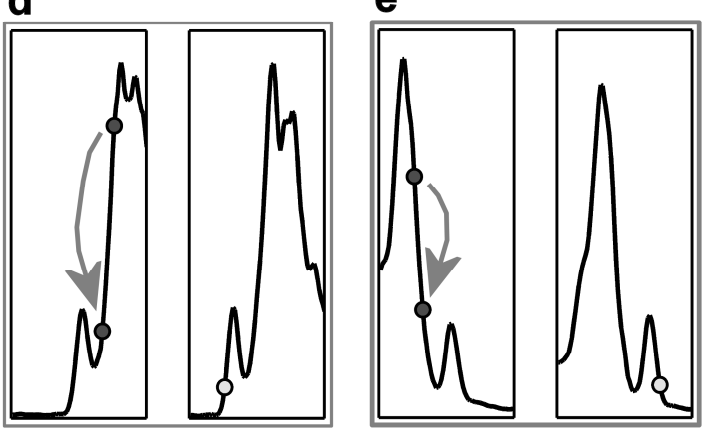

f

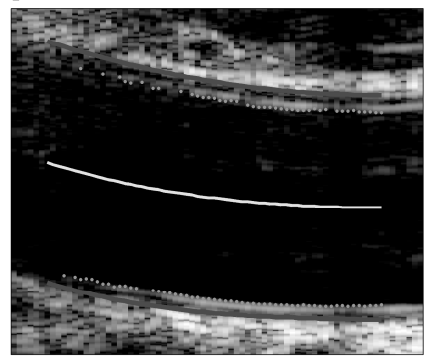

Fig. 6.3 (color version on page 152) - a) MAB method acting across one vertical line; top panel: near wall; bottom panel: far wall; the initial seed points are placed at $2 \mathrm{~mm}$ from the CCA centreline. In these black-and-white figures, the evaluation domain (white circles) changes size at each iteration according to the previously detected adventitial position. The smoothing domain (grey circles, here shown only at the first iteration for visual convenience) extends $716 \mu \mathrm{m}$ in depth and $945 \mu \mathrm{m}$ in width. The thicker circles/points refer to the final iteration. b) The algorithm acts on the 61 centremost signals in the ROI. c) Intimal contour outliers are rejected. In this example the IMT unbiasing procedure involves equalization, subsequent to re-alignment and lateral averaging, for far wall adventitia ( $d$, left panel) with respect to far wall intima (d, right panel), and for near wall adventitia (e, left panel) with respect to near wall intima (e, right panel). f) Final result of the automatic CCA segmentation.

The multiscale nature of our approach refers to the fact that the evaluation domain is resized in each iteration step according to its current position with respect to the adventitial wall contour (Fig 6.3a). Starting from the second iteration the evaluation domain ends at $1 / 4$ of the axial system resolution (i.e. $83 \mu \mathrm{m}$ ) ahead of the adventitial border to avoid that relatively bright adventitial echoes interfere with intima detection. The equilibrium state of each point in the contour is reached when the contour point obtained at the $i$ th iteration is the same as the one determined either in the $(i-$ 1)th or (i-2)th iteration (Gemignani et al. 2004). 


\subsection{6 - Diameter and IMT estimation: automated post processing}

After $M A B$, each wall presents both adventitial and intimal contour estimates. The internal CCA contour estimated with $M A B$ may be locally inconsistent with the real lumen-intima interface (Fig. 6.3b), because of luminal reverberations and artefacts nearby the contour, a too weak (or even not present) intima due to an improper CCA scan plane, or a very reflective adventitial echo leaking into the MAB kernel. To reject outliers we discard contour points for which the IMT deviates more than 165 $\mu \mathrm{m}$ (i.e., half the US axial resolution) from the median IMT value, independently for each wall (Fig. 6.3c).

At this stage the observed IMT value may be biased by the fact that the methods used to detect the adventitial and intimal borders are different in nature, producing their respective detection points at different levels relative to the respective echo peaks. To remove the associated bias, the adventitial echo envelopes are separately realigned for each CCA wall according to the respective detected adventitial contour position, and averaged in the lateral direction. The same procedure is repeated for the IMT envelope segments. The results of this procedure are, for each wall, two slightly different lateral averages centered on the adventitia and intima contours, respectively (Fig. 6.3d and 6.3e). Next, for both lateral averages the relative mean echo amplitude at the detection point with respect to the corresponding (intimal or adventitial) echo peak is computed. The detected boundary positions are then equalized in relative echo amplitude, based on the one in which the contour-topeak echo level ratio is smaller (ratio computed after subtraction of the average lumen echo level). In the large majority of cases, this unbiasing procedure displaces for both walls the adventitial contour towards the lumen (Figs. 6.3d-f).

External diameter, internal diameter and both near wall and far wall intima-media thicknesses can be derived from the contour positions on a frame-by-frame basis. The distances are computed perpendicularly to the vessel wall rather than along the US beams, taking into account the CCA local centerline orientation. The IMT of both near and far wall, after orientation compensation, is averaged across the ROI for each wall separately. The internal (intima-intima) CCA diameter can be obtained in two ways. The first one is computing the difference between far wall and near wall intima positions for only those echo lines without IMT gaps on both walls, compensating each line-estimate for the local CCA centerline orientation and then averaging across the ROI. The second way averages instead the intimal positions at each wall, subtracts the near wall average from the far wall average, and finally compensates for the mean CCA orientation in the frame. 
a

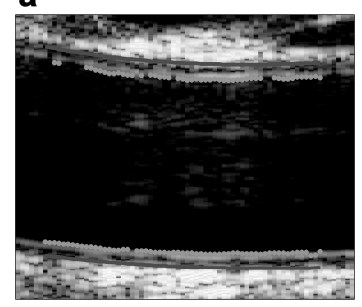

d

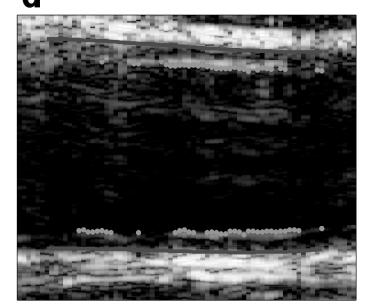

g

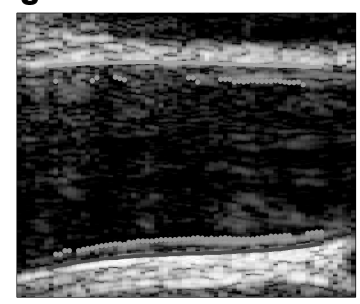

b

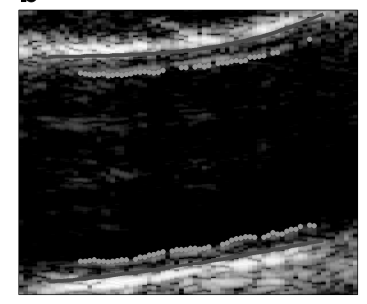

e

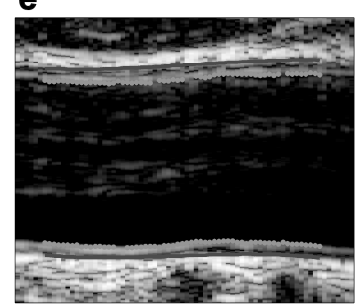

$\mathbf{h}$

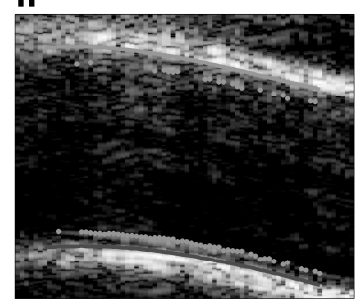

C

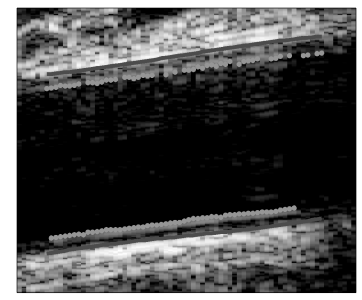

f
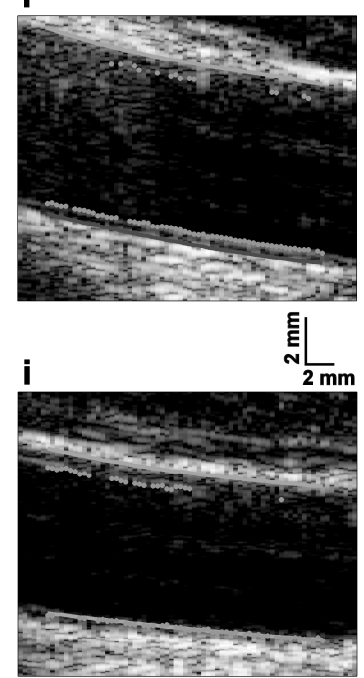

Fig. 6.4 (color version on page 153) - Examples of automatic CCA segmentation. In these black-and-white figures, an "in-plane" condition is symbolized by intimal and adventitial contours of different color. When the automatic scan plane control yields an "out plane" result, both contours have the same color.

The frame-specific adventitial contour displacement, due to the automatic IMT unbiasing procedure, may affect the smoothness of the external diameter in time. That is why the external diameter waveform, unlike the IMT waveform, is not computed by subtracting the bias at each frame, but rather by subtracting from the original external diameter waveform the average difference induced by the unbiasing procedure (average computed for those frames where both CCA walls are in plane). Fig. 6.4 shows some examples of CCA segmentation, whereas Fig. 6.5 shows diameter and IMT waveforms (sampled at $30 \mathrm{~Hz}$, i.e. the B-mode frame rate). 


\subsection{7 - Automatic scan plane control}

The intimal echogenicity in the US image highly depends on the angle of incidence of the US beam, which has to be perpendicular for an optimal view. Due to artery or probe motion during the scan, the perpendicularity condition may be temporarily lost, resulting in intima signals that are not distinct from the adventitial or from the luminal echo level. This might lead to a large number of missing IMT values. Rather than trying to deduce in such cases the intima position from previous or subsequent frames, we chose to label per frame each wall as "in plane" or "out of plane", coding the information by contour colors (Fig. 6.4). In order for either near or far CCA wall to be labeled as "in plane" in a specific frame, all following conditions have to be satisfied:

1) The maximal difference between the adventitial contours orientation must be less than 15 degrees, consistent with the observation that tapering hardly exceeds 10 degrees.

2) For each wall the percentage of intimal points discarded by outlier rejection must be less than $50 \%$. A higher percentage of outliers would signify a very low lateral consistence of the intimal echo, possibly due to an improper scan plane.

3) Each detected intimal contour point (after IMT unbiasing) should always be closer to the lumen than the corresponding adventitial contour point (on the same echo line and for the same wall).

4) The average IMT calculated after post processing must exceed $165 \mu \mathrm{m}$. Given the US axial resolution of the US system used $(330 \mu \mathrm{m})$, it is very likely that IMT values smaller than half such resolution are erroneous and/or speckle-dependent.

When condition 1 is not satisfied on either wall, both walls are labeled "out of plane". Conditions 2, 3 and 4 are instead wall-specific, i.e. they pertain only to one wall. Figs. $6.4 \mathrm{f}-\mathrm{h}$ show examples in which the near wall is out of plane (condition 2 is not satisfied). Rejection of "out of plane" wall estimates based on conditions 3 and 4 is facilitated by the IMT unbiasing procedure. When a wall is not properly visualized, the intimal peak will be too weak and hence overwhelmed by the adventitial echo. The IMT unbiasing procedure results then in an adventitial contour too close to the intimal contour, or even in a common value if only one peak is present (Fig. $6.4 i)$.

\subsection{8 - Accuracy}

To evaluate the accuracy of the proposed algorithm, and to test the adequacy of the automatic IMT unbiasing procedure, synthetic ultrasound images were generated based on realistic RF signals. We acquired an RF signal from a single reflector, 
namely an $80 \mu \mathrm{m}$ thick nylon wire submerged in water at a focal depth of $20 \mathrm{~mm}$, with the same US probe and scanner as used for the CCA acquisitions.
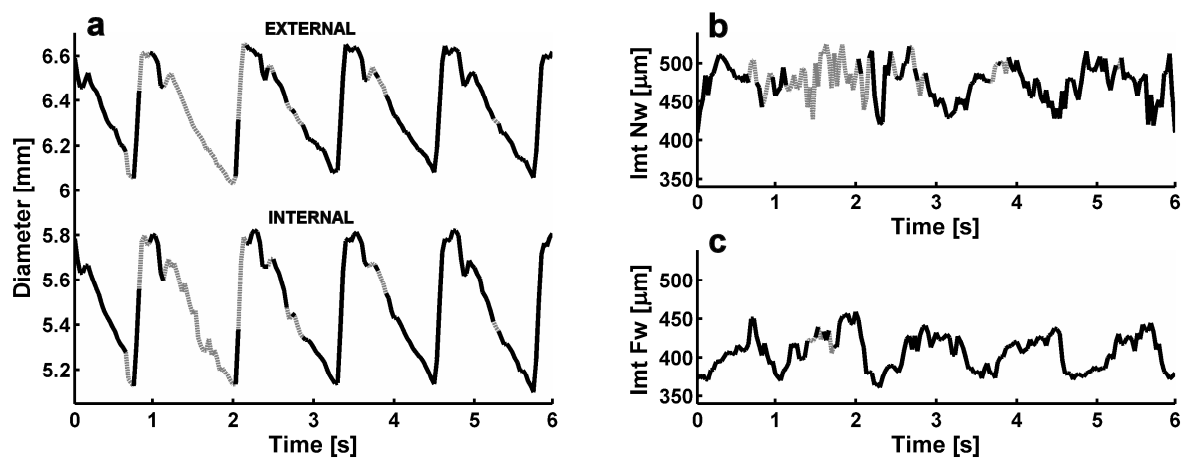

Fig. 6.5 - a) External (adventitia-adventitia) and internal (intima-intima) diameter waveforms. b) Near wall IMT waveform. c) Far wall IMT waveform. The B-mode frame rate ( $30 \mathrm{HZ})$ is also the waveform sampling rate. The black solid sections in the waveforms refer to the "in plane" condition, the grey dashed sections to "out of plane". The diameter is considered "in plane" when both walls are.

The normalized RF pulse (Fig. 6.6a) has a full width at half maximum of $300 \mu \mathrm{m}$, and it presents a small secondary trailing lobe with a peak of $-22 \mathrm{~dB}$ with respect to the main lobe peak. The distance between both peaks is $350 \mu \mathrm{m}$. The acquired RF pulse is artificially oversampled at $2.5 \mathrm{GHz}$. Displacing replicas of the oversampled RF signal in time, scaled to typical adventitia and intima echo amplitudes derived empirically, and summing them, creates synthetic CCA RF signals. Oversampling is necessary in order to have a high definition in imposed intima and adventitia positions. The summed signal is then decimated to the sampling frequency of the US scanner (33.3 MHz), in order for the segmentation algorithm to act on data compatible and similar to the in vivo situation. The external CCA diameter, defined as the distance between the near wall adventitia trailing edge and the far wall adventitia leading edge, is fixed to $6.5 \mathrm{~mm}$. The IMT on both walls is varied between 300 and $800 \mu \mathrm{m}$ with an increment of $50 \mu \mathrm{m}$; the internal diameter varies accordingly. Images are then generated, replicating the RF envelopes in lateral direction 74 times. Figs. 6.6b and $6.6 \mathrm{c}$ show the simulated CCA RF signal and image, respectively, for an imposed IMT of $500 \mu \mathrm{m}$. The imposed intima peak level, based on average in-vivo empirical findings, is at $-12.4 \mathrm{~dB}$ with respect to the adventitial one on the near wall, whereas on the far wall it is set at $-13.8 \mathrm{~dB}$. A more extensive evaluation of the accuracy for varying intimal echo levels can be found in Appendix 6B. 


\subsection{9 - Statistical analysis}

The intra-subject intra-recording precision is evaluated by averaging the intrarecording beat-by-beat variance for diastolic and mean values of external diameter (Figs. 6.7a-b), internal diameter (Figs. 6.7c-d), far wall IMT (Figs. 6.7g-h) and near wall IMT (Figs. 6.7i-I). The "in plane" and "out plane" average percentages are also evaluated (Figs $6.7 \mathrm{e}, 6.7 \mathrm{f}, 6.7 \mathrm{~m}, 6.7 \mathrm{n}$ ). The diastolic values are point-values in time, so they can be directly associated to an "in plane" or "out plane" condition, both for IMT (condition checked for one wall) and diameter (condition checked for both walls simultaneously). Mean values refer instead to a complete beat, to which a quality flag is assigned using the following criteria:

- A beat in the IMT waveform is considered "in plane" when at least $50 \%$ of the samples in the beat refer to US frames in which the respective wall (near or far) is labelled "in plane";

- A beat in the diameter waveform (either internal or external diameter) is considered "in plane" when at least $30 \%$ of the samples in the beat refer to US frames where both CCA walls are labelled "in plane".

Intra-subject inter-recording variations are evaluated with Bland-Altman plots (Figs 6.8 and 6.9) depicting the differences between intra-recording and intra-subject averages. Observed variations in external diameter and far wall IMT are compared with those of the ART.LAB system.

\section{4-Results}

\subsection{1 - Accuracy for synthetic ultrasound images}

Figs. 6.6d and 6.6e show for both far and near wall the IMT estimation error as function of the imposed IMT values, prior to and after IMT unbiasing. The average percentage deviation from the true IMT value prior to unbiasing is similar for both walls (Table 6.1). The unbiasing procedure changes the IMT deviation from an overestimation of $8.5 \%$ to an underestimation of about $3 \%$. The external diameter estimation is marginally affected by IMT unbiasing, whereas internal diameter estimation is not (Table 6.1). 

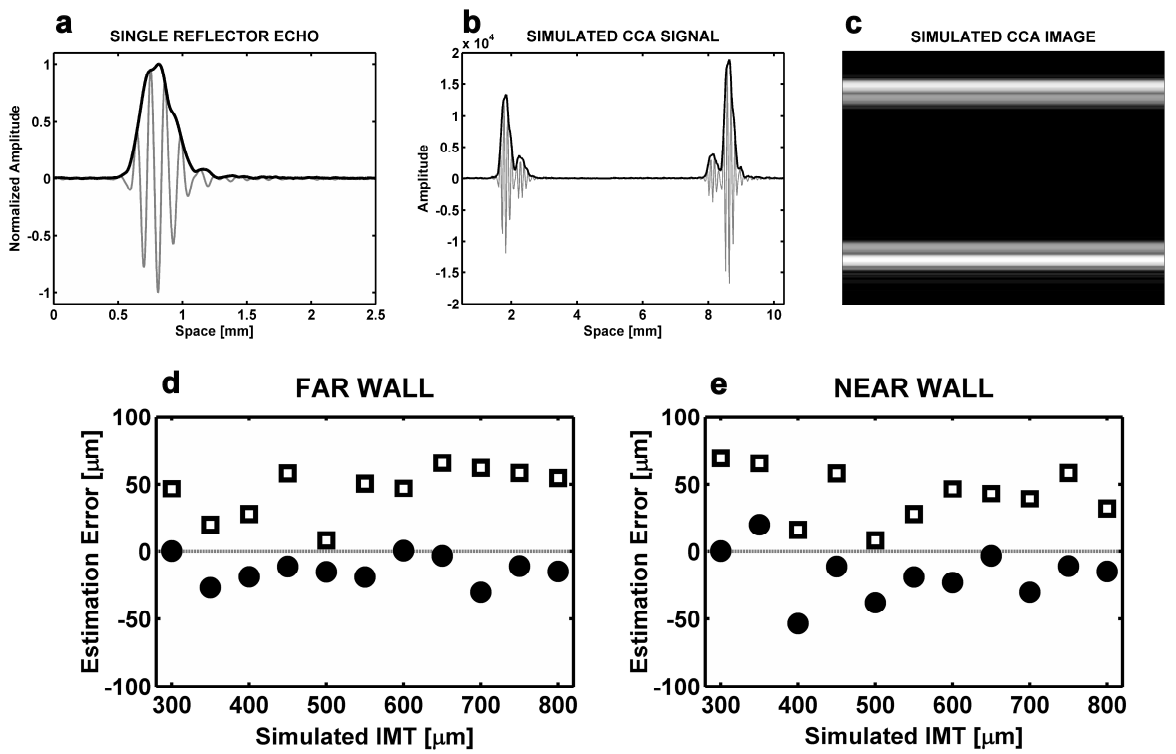

Fig. 6.6 - Accuracy evaluation performed on synthetic images. a) Normalized RF pulse (oversampled at $2.5 \mathrm{GHz}$ ), showing a small secondary trailing lobe. By scaling and displacing the pulse in shape and position, synthetic CCA RF signals (b) and images (c) are generated (in this example, the external diameter is $6.5 \mathrm{~mm}$, and the imposed IMT is $500 \mu \mathrm{m}$ for both walls). IMT estimation errors obtained with the MAB method for far (d) and near wall (e) are on average lower with IMT unbiasing procedure (black circles) than without (white squares). On the near wall, the presence of the trailing lobe affects estimation results up to $500 \mu \mathrm{m}$ due to RF phase interference.

Table 6.1 - Average deviations from true values [\%]

\begin{tabular}{lll}
\hline & Deviation \% pre-unbiasing & Deviation \% post-unbiasing \\
IMT Far Wall & $+8.5 \%$ & $-2.6 \%$ \\
IMT Near Wall & $+8.8 \%$ & $-3.0 \%$ \\
External Diameter & $+0.5 \%$ & $-1.3 \%$ \\
Internal Diameter & $-1.0 \%$ & $-1.0 \%$ \\
\hline \hline
\end{tabular}

The IMT-unbiasing procedure improves accuracy for intimal and adventitial contours.

\subsection{2 - In vivo evaluation}

The algorithm is tested on 36 US recordings from 12 healthy subjects, for a total of 6480 frames analyzed. Fig. 6.4 shows several examples of the performed automatic MAB segmentation. The quality of the contour overlays is visually checked using movies. On a general purpose PC (Intel Celeron $3 \mathrm{GHz}, 448 \mathrm{MB}$ of RAM) the average processing time using MATLAB for one recording consisting of 180 US frames, including data loading and post processing, is 4 minutes and 30 seconds (i.e., an aver- 
age of 1.5 seconds for each US frame). The MAB reaches equilibrium after on average 6 and 7 iterations for the far and near wall of the CCA, respectively. At equilibrium the size in depth of the evaluation domain is on average $1 \mathrm{~mm}$ for the far wall and $1.2 \mathrm{~mm}$ for the near wall. Realistic diameter and IMT waveforms are extracted for each US recording (Fig. 6.5), despite a low B-mode frame rate (30 fps). In order to retain the sharp changes in diameter and IMT waveforms during the systolic phase, no smoothing in time is performed.

\subsection{3 - Precision}

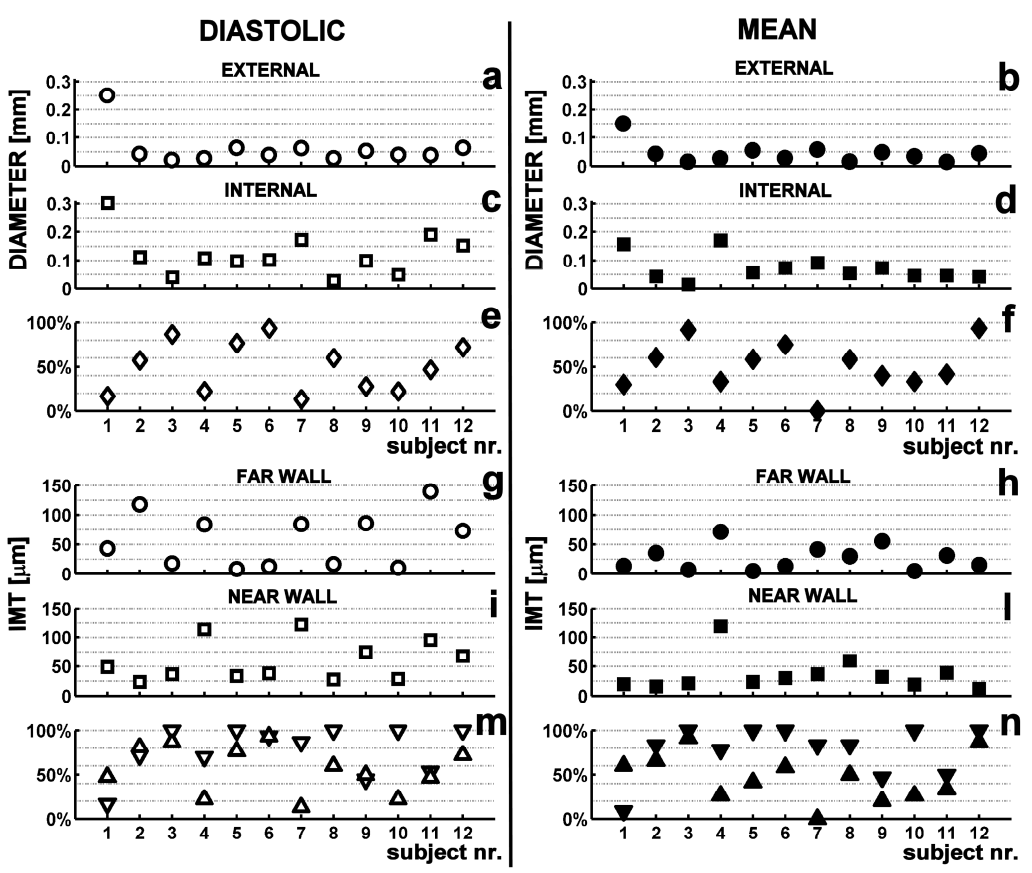

Fig. 6.7 - Intra-subject intra-recording standard deviation evaluated on a beat-to-beat basis. a) external diastolic diameter; b) external mean diameter; c) internal diastolic diameter; d) internal mean diameter; e) average percentage of "in plane" diastolic diameter points; f) average percentage of "in plane" diameter beats; g) far wall diastolic IMT; h) far wall mean IMT; i) near wall diastolic IMT; I) near wall mean IMT; $m$ ) average percentage of "in plane" diastolic IMT points; n) average percentage of "in plane" IMT beats. In panels $m$ ) and n), upwards-pointing triangles refer to the near wall, whereas downwards-pointing triangles refer to the far wall.

The intra-recording precision for the external diameter is on average less than 50 $\mu \mathrm{m}$, both for diastolic and mean beat values (Figs. 6.7a-b). Only subject \#1 shows a much higher variability, due to a consistent out of plane condition. The intrarecording precision for the internal diameter is better for mean beat values (standard deviation on average smaller than $100 \mu \mathrm{m}$ ) than for diastolic ones (Figs. 6.7cd). Diastolic IMT values are sometimes quite difficult to assess reliably at a low 
frame update rate, as shown by the high intra-recording variabilities (Figs. 6.7g-i). Averaging all the values over one beat decreases significantly the standard deviation of the estimates (Figs. 6.7h-I), to values on average lower than $50 \mu \mathrm{m}$ for both walls.
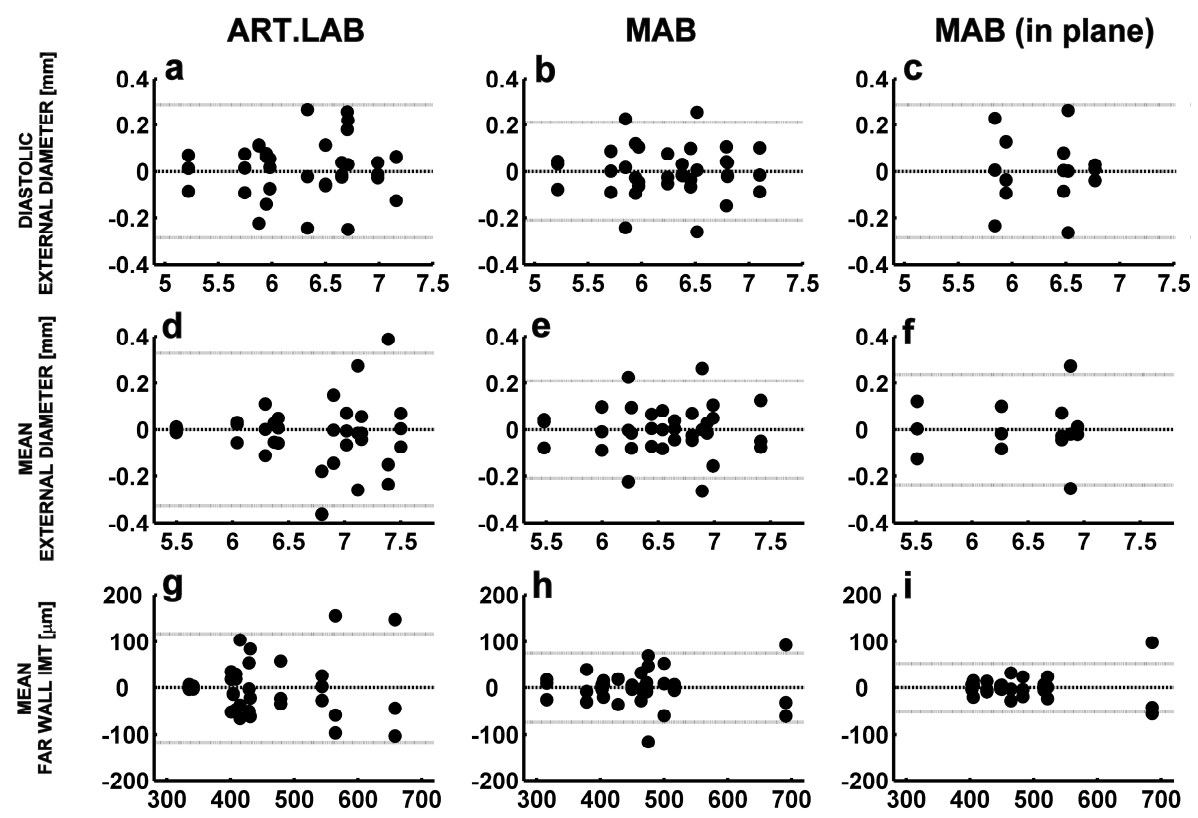

Fig. 6.8 - Intra-subject inter-recording Bland-Altman plots (beat-to-beat) for diastolic external diameter $(a, b, c)$, mean external diameter $(d, e, f)$ and mean far wall IMT $(g, h, l)$. Left column: ART.LAB; middle column: MAB method, all beats; right column: MAB method restricted to "in plane" beats. The grey lines refer to twice the standard deviation.

The inter-recording Bland-Altman plots (Fig. 6.8) compare the proposed MAB method with the ART.LAB system for diastolic external diameter, mean external diameter and mean far wall IMT. Inter-recording variations in diameter and IMT values can be due to variability in the estimation procedure or also (and more often) to slight differences in probe positioning between acquisitions. Even though the results for the two systems are in good accordance, MAB shows a better precision than ART.LAB does (middle column versus left column in Fig. 6.8) with standard deviations that are on average $50 \mu \mathrm{m}$ lower for diameter estimates and $25 \mu \mathrm{m}$ lower for IMT. On average the MAB method presents a beat-to-beat inter-recording standard deviation in the order of $100 \mu \mathrm{m}$ for external diameter (Fig. 6.8) and 115 $\mu \mathrm{m}$ for internal diameter (Fig. 6.9). Considering only the "in plane" beats significantly improves the precision for far wall IMT from $40 \mu \mathrm{m}$ to $25 \mu \mathrm{m}$ (Fig. 6.8i), whereas it does not for the external diameter (Figs. 6.8c, 6.8f). Fig. 6.9 shows interrecording Bland-Altman plots for mean internal diameter (6.9a-b) and mean near wall IMT (6.9c-d). Again the "in plane" condition improves the beat-to-beat preci- 
sion of IMT estimates from $50 \mu \mathrm{m}$ to $30 \mu \mathrm{m}$, but not for the diameter estimates. The percentages of "in plane" points in Figs 6.8 and 6.9 with respect to the "all beats" plots (36 recordings) are: $42 \%$ for diastolic and mean external diameter, $72 \%$ for far wall IMT, $42 \%$ for mean internal diameter and $25 \%$ for near wall IMT.
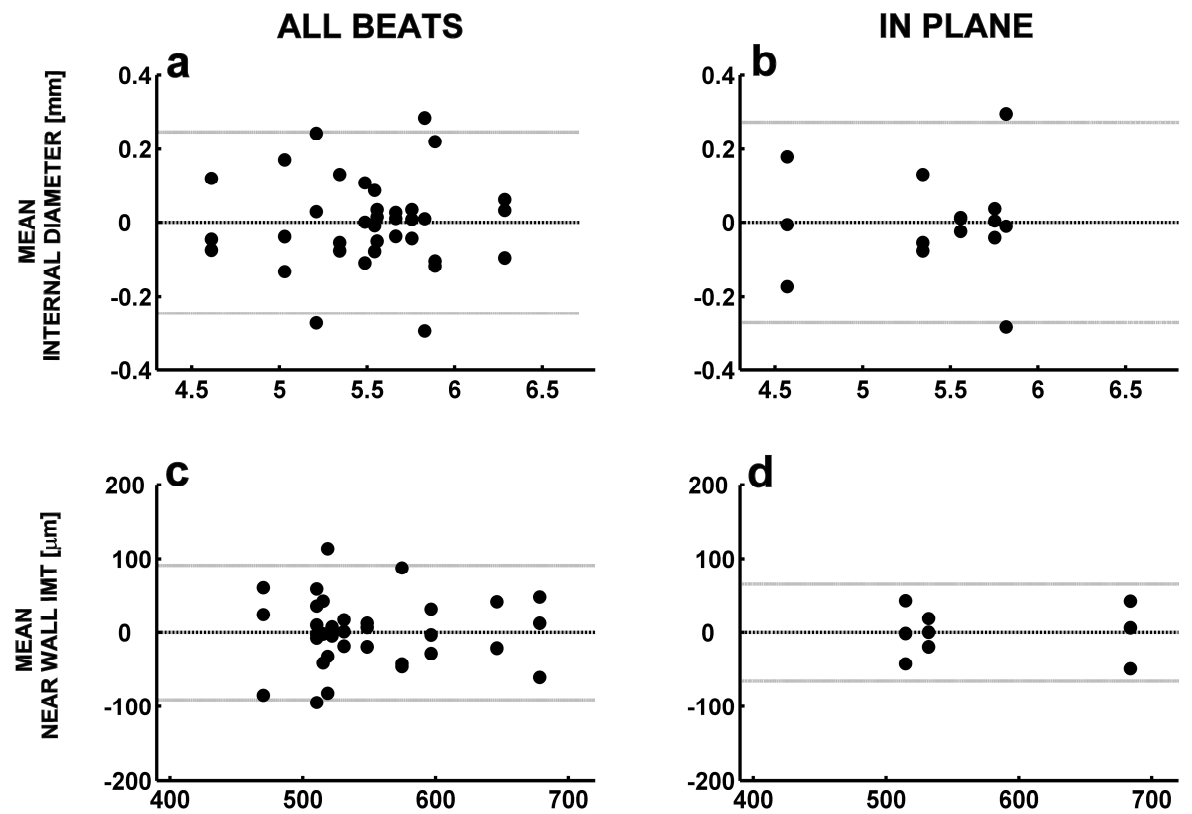

Fig. 6.9 - Intra-subject inter-recording Bland-Altman plots (beat-to-beat) for mean internal diameter ( $a, b)$ and mean near wall IMT (c, d) estimated with the MAB method. Left column: all beats; right column: only "in plane" beats. The grey lines refer to twice the standard deviation.

\section{5 - Discussion}

The automatic CCA segmentation performed with the proposed method, combining sustain-attack filter, multiscale anisotropic barycenter (MAB) and dedicated post processing, is robust and reliable, and shows a high accuracy and precision for the estimation of CCA external diameter, internal diameter and IMT on both near and far CCA walls. Our implementation does not require human interaction, since the positions of both near and far lumen-intima interfaces are estimated starting from an automatically retrieved lumen centerline instead of using a manually initialized approximated intimal contour. The multiscale iterative optimization of the evaluation domain size makes the MAB method insensitive to the initial distance between the starting seed points, placed in the vicinity of the CCA centerline, and the arterial wall-lumen interface to localize. At equilibrium the size of the evaluation domain still exceeds the size of the concentric smoothing domain, as theoretically required 
in order for the barycenter to maintain its desirable edge detection properties (Demi 2001).

The far wall and the near wall of the CCA exhibit different segmentation results. Figs. $6.5 \mathrm{~b}$ and $6.5 \mathrm{c}$ show that in some subjects the near wall IMT can be systematically higher than the far wall IMT, with differences reaching $50 \mu \mathrm{m}$ or more. On the other hand, in synthetic images the difference between far wall and near wall IMT (Figs $6.6 \mathrm{~d}$ and $6.6 \mathrm{e}$ ) reaches up to $30 \mu \mathrm{m}$ in magnitude, but with varying sign depending on the imposed IMT value. This discrepancy can be explained by the fact that the echo used for the accuracy analysis is based on a single specular reflection in water, whereas the adventitia in the CCA is a complex and multilayered structure that produces a composite reflection, and therefore its trailing edge behaves differently. We are, however, confident that the synthesis approach is more realistic in terms of accuracy analysis than those obtained by imaging an arterial-like structure in tissue mimicking gel, mainly because the dimensions of the arterial layers in such a phantom are very difficult to control and to measure precisely (Potter 2008). The oscillations in estimation errors for the near wall (Fig. 6.6e), exhibited in the IMT range between 300 and $500 \mu \mathrm{m}$, are due to the phase interference between the trailing ringing lobe of the adventitial RF signal (spaced at $350 \mu \mathrm{m}$ from the main adventitial peak, see Fig. 6.6a) and the superimposed intimal main echo. We confirmed this assumption by performing the same phantom images analyses gradually decreasing the intimal echo amplitudes with respect to the adventitial ones, both on near and far CCA wall (see Appendix 6B). This demonstrates that on the near wall phase interference between the adventitial and the intimal echo affects the appearance of the RF envelopes (speckle), confirming the notion (Touboul 2007) that the IMT should be measured preferably on the far wall, especially for an IMT less than twice the system resolution. Figs. $6.5 \mathrm{~b}$ and $6.5 \mathrm{c}$ confirm that the far wall IMT waveform appears much more smooth and realistic than the near wall one.

Table 6.1 clearly illustrates that the IMT unbiasing procedure improves the accuracy of IMT estimates for both walls at the expense of the accuracy of the external diameter. The latter remains acceptable because its value remains within a $2 \%$ range from the real one. Basically the IMT unbiasing procedure uniforms deviations of intimal and adventitial contours, which are initially different (and possibly opposite in sign) because of the different methods used for delineation, i.e. the sustainattack filter for adventitial position and $M A B$ for intimal contour. The observed small oscillations of IMT estimation error are negligible compared with CCA diameter values. Therefore, the proposed algorithm is well suited for estimation of both internal and external CCA diameter (Fig. 6.5), even though the update rate is limited to $30 \mathrm{fps}$. The internal diameter and IMT waveforms are less smooth than those of the external diameter because speckle affects the intima position more than the 
adventitia position. Fig. 6.5 also demonstrates that the proposed method for automatically labeling US frames as "in plane" or "out of plane" is consistent with the appearance of internal diameter and IMT waveforms, which generally contain higher noise levels in the "out of plane" sections. It is usually more difficult to have an "in plane" condition on the near wall, as shown in Figs. 6.4f-h. Occasionally, due to probe and/or artery movements, the perpendicular insonation incidence can be temporarily lost for both walls (Fig. 6.4i). In a real-time application, the sonographer could employ visual feedback about the "in plane" condition to optimize the incidence angle between US beam and arterial walls.

The internal and external diameter, both for diastolic and mean beat values, can be obtained with a good intra-recording precision (Fig. 6.7). The internal diameter precision is affected by the scan quality control measure, unlike the external diameter precision. This means that the "in plane" criteria (section 6.3.7) most commonly violated are nr. 2, 3 and 4 . The relation between the average amount of "in plane" beats and the intra-recording precision is better reflected in the mean beat values, e.g. subjects nr. 1, 4 and 7 show higher inner diameter variations in combination with lower percentages of "in plane" beats (Figs. 6.7d, 6.7f).

In some subjects, diastolic IMT (Figs. 6.7g, 6.7i) can have poor intra-recording precision. This is expected, however, due to the low temporal resolution $(30 \mathrm{~Hz})$ of $\mathrm{B}$ mode ultrasound and due to the speckle pattern affecting intimal measurements. Averaging over a beat improves IMT precision (Figs. 6.7h, 6.7l) to intra-recording variations lower than $50 \mu \mathrm{m}$. Except subject nr.1, whose consistent "out of plane" condition results in constant but wrong IMT values, all other subjects with low far wall intra-recording variations in IMT, both for diastolic and mean values, exhibit a very high "in plane" rate. The same relation is observed for near wall estimates although less consistently. The IMT precision in combination with the "in plane"/"out of plane" labeling proves, therefore, its potential for real-time situations.

Figs. 6.8 and 6.9 show that the inter-recording standard deviations of the estimates are higher than the intra-recording ones, caused by slight variations of probe position and/or orientation on different acquisitions within the same subject. However, the results show a good repeatability for the MAB method, which exhibits a lower variation of the estimates than the ART.LAB system (Fig. 6.8). Figs. 6.8i and 6.9d indicate that when considering only the "in plane" beats, the IMT inter-recording variations decrease significantly, both for near and far wall. The same doesn't hold for external and internal diameter, suggesting that for these measures physiological and/or operational variations independent of scan quality are preponderant. Another issue worth considering is the fact that getting both the far wall and the near 
wall "in plane" simultaneously can be quite difficult, especially due to the echo characteristics of the near wall. Hence, the amount of beats with both walls properly imaged can be low, even when the recording quality of the far wall is good.

Computing the internal diameter either based on line-by-line correspondence or by averaging the intimal wall positions does not significantly affect the intra-recording precision (the figures related to the internal diameter in this chapter always refer to the first modality). Also the intra-recording diameter averages are not strongly influenced by the methodology. However, the subjects analyzed in this study are young and healthy volunteers in which the CCA is imaged close to perpendicularity. If the CCA orientation is far from perpendicular or more irregular, as could be the case for elderly patients, the line-correspondence method for the calculation of internal CCA diameter is advisable. We also noticed from the inter-recording BlandAltman plots that the standard deviation values obtained by averaging the contour position across a frame prior to diameter computation are slightly lower than the ones shown in Figs. 6.9a-b. However, this decrease is not indicative of a better estimation technique, but should be rather attributed to the higher number of intimal contour points considered and to the smoothing of the potentially varying centerline orientation across the frame.

\section{6-Conclusion}

We introduced a method for the automatic segmentation of the common carotid artery in longitudinal ultrasound B-mode scans that provides precise and robust delineation of lumen-to-intima and media-to-adventitia interfaces on both common carotid artery walls. The method also provides a visual scan quality feedback aimed to signal the sonographer that the insonation plane is not adequate. The algorithm functions without user interaction. It consists of a combined approach involving automatic CCA recognition, sustain-attack filter, "Multiscale Anisotropic Barycenter" (based on the first absolute central moment), and knowledge-based post processing. The method was tested on 36 ultrasound recordings from 12 healthy subjects, and on synthetic images. The algorithm successfully delineates intimal and adventitial layers regardless of local orientation and curvature of CCA walls, while its programming structure and computation times are suited for future real-time implementation. Results show high accuracy and precision for external CCA diameter, internal CCA diameter, far wall IMT and near wall IMT, indicating robustness and clinical applicability. Further developments for the algorithm could involve the automatic recognition and analysis of plaques, based on geometrical and/or texture features. 


\section{Appendix 6A-Barycenter of the echo level deviation}

The original edge detector, as suggested by Gemignani (2004), is hereby provided. $f(w, d)$ is the discrete echo level map of an ultrasound image encoded in grey levels, where $w$ and $d$ represent width and depth in sample points, respectively, and $\Omega_{1}$ and $\Omega_{2}$ (Fig. 6.10) are two concentric circular domains with radii $r_{1}$ and $r_{2}$ defined as

$$
\Omega_{i}=\left\{(k ; l): \sqrt{k^{2}+l^{2}} \leq r_{i}\right\} \quad i=1,2
$$

where $k$ and $/$ are the coordinates of a generic sample with respect to a Cartesian plane originating in $\mathbf{p}[w, d]$ (Fig. 6.10).

Every point of the domain $\Omega_{2}$ can be associated to a mass $h(\mathbf{p}, k, l)$, defined as

$$
h(\mathbf{p}, k, l)=\left|f(w-k, d-l)-f_{1}(\mathbf{p})\right| g\left(k, l, r_{2}\right), \quad(k, l) \in \Omega_{2}
$$

where

$$
f_{1}(p)=\sum \sum_{(k, l) \in \Omega_{1}} f(w-k, d-l) g\left(k, l, r_{1}\right)
$$

is a smoothed version of $f$ in the domain $\Omega_{1}$, and $g\left(k, l, r_{i}\right)$ is a normalized Gaussian weight function with standard deviation $\sigma_{i}=r_{i} / 3$, ensuring that the output of the smoothing filter depends continuously on the input data.

Given the definition of mass in equation (6.2), the "first absolute central moment" can be defined so that

$$
\mathrm{e}(\mathbf{p})=\sum \sum_{(k, l) \in \Omega_{2}} \mathrm{~h}(\mathbf{p}, \mathrm{k}, \mathrm{l})
$$

$e(\mathbf{p})$ is a statistical filter that measures the variation of the echo level with respect to the local mean. The first order is chosen to have maximum freedom of choice for the sizes $\Omega_{1}$ and $\Omega_{2}$ in relation to enhancement of discontinuities, and also to ensure that the filter involves the lowest integer power, which is a desirable property for dealing with noise (Demi 2001). The mass center (or "barycenter") b of $h(\mathbf{p}, k, l)$ can be computed as 


$$
\mathbf{b}(\mathbf{p})=\left\{\begin{array}{ccc}
\sum_{(k, l) \in \Omega_{2}} \mathrm{~h}(\mathbf{p}, \mathrm{k}, \mathrm{l}) \boldsymbol{\Gamma} & & \\
\mathrm{e}(\mathbf{p}) & \text { if } & \mathrm{e}(\mathbf{p}) \neq 0 \\
0 & \text { if } & \mathrm{e}(\mathbf{p})=0
\end{array}\right.
$$

where $\boldsymbol{\Gamma}$ is a vector with components $(-k,-l)$. Vector $\mathbf{b}$ (Fig 6.10$)$ joins point $\mathbf{p}$ to the mass centre of the echo level deviation in the domain $\Omega_{2}$. It is demonstrated (Demi 2001) that $\mathbf{b}$ has two very useful properties when $\Omega_{2}$ contains a grey level discontinuity: it is perpendicular to the discontinuity, and the located barycenter is always closer to the discontinuity than $\mathbf{p}$, regardless of the distance between $\mathbf{p}$ and the discontinuity. These features make this approach particularly suited for edge detection, not only in ultrasound but also in other imaging modalities.

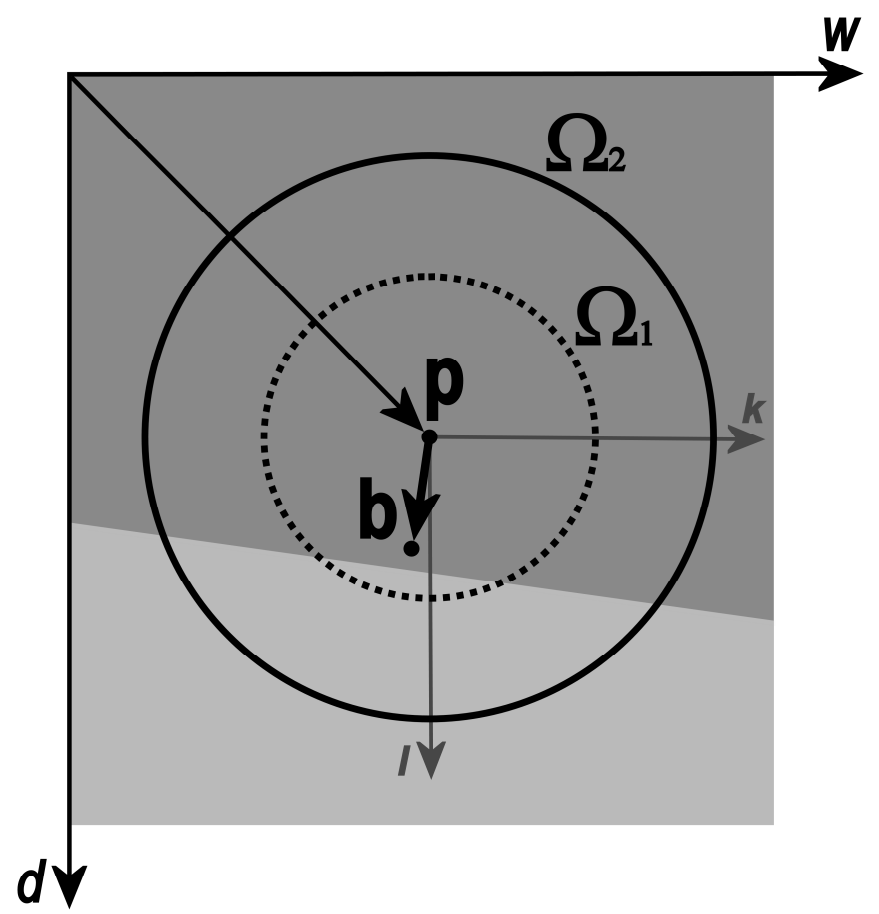

Fig. 6.10 - Schematic representation of the "barycenter" method. Vector $\mathbf{b}$ joins point $\mathbf{p}$ to the barycenter of the grey level deviations in domain $\Omega_{2}$ with respect to the average value in domain $\Omega_{1}$. The detected point is located between $\mathbf{p}$ and the grey level discontinuity. 


\section{Appendix 6B - Echo interference and near wall IMT estimation}
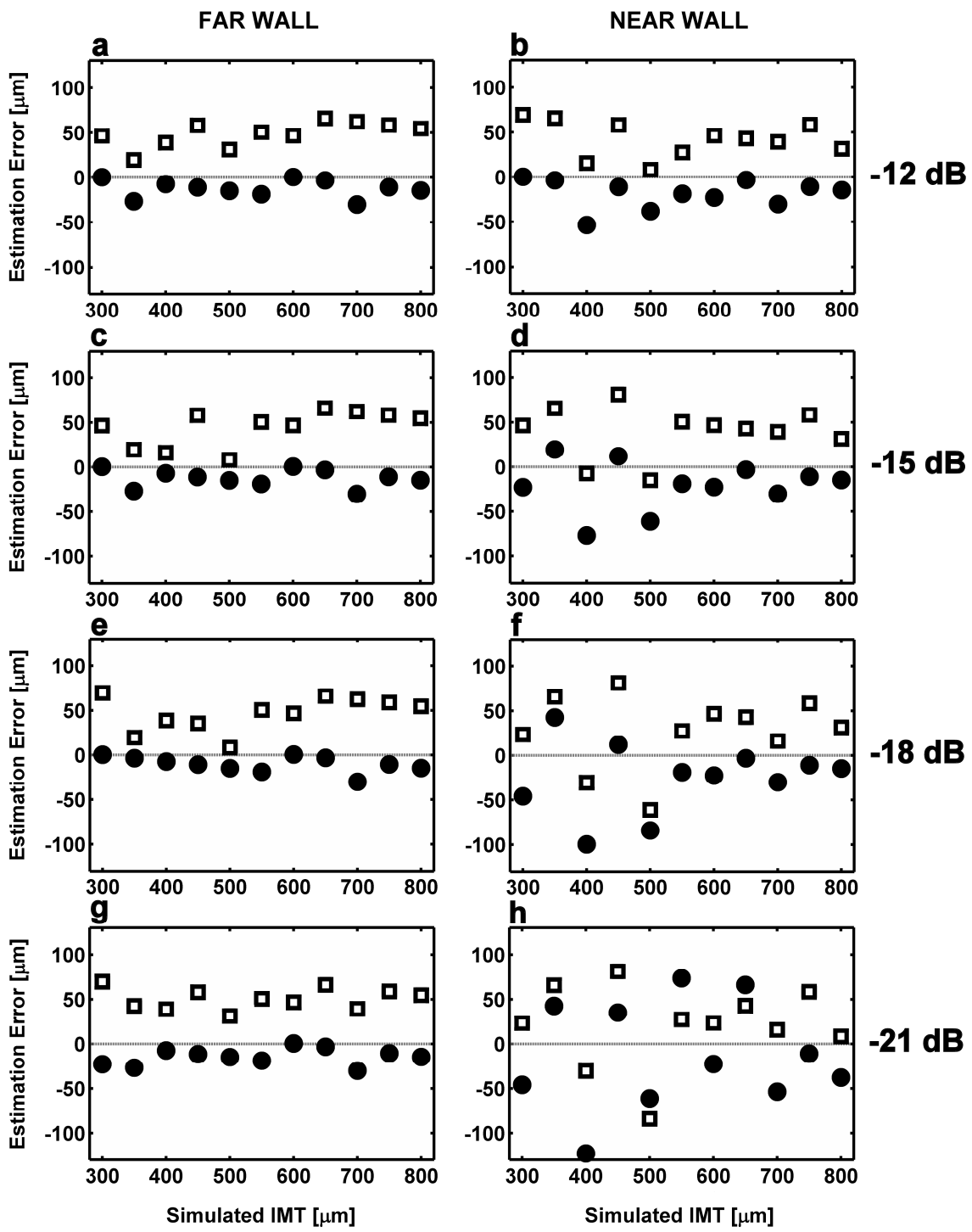

Fig. 6.11 - IMT estimation errors obtained with the MAB method in synthetic CCA US images for both near (left column) and far wall (right column) at an intima-to-adventitia echo level ratios of $-12 d B(a, b),-15 d B(c, d),-18 d B(e, f)$ and $-21 d B(g, h)$. With the IMT unbiasing procedure (black circles) the error is lower than without it (white squares). On the near wall adventitia-intima echo interference affects the estimation results, whereas it does not for the far wall. 
Synthetic CCA RF signals and images (similar to the ones depicted in Fig. 6.6) were generated using gradually varying the intima-to-adventitia echo amplitude ratios and a fixed external diameter of $6.5 \mathrm{~mm}$. For each case $(-12 \mathrm{~dB},-15 \mathrm{~dB},-18 \mathrm{~dB}$ and $-21 \mathrm{~dB}$ ) the external diameter was fixed to $6.5 \mathrm{~mm}$, and the IMT on both CCA walls was varied between 300 and $800 \mu \mathrm{m}$ in steps of $50 \mu \mathrm{m}$. As depicted in Figs. 6.11a, $6.11 \mathrm{c}, 6.11 \mathrm{e}$ and $6.11 \mathrm{~g}$, the estimation error pattern for the far wall as function of the IMT is rather independent of intimal echo amplitude. In contrast, decreasing the strength of the intimal echo on the near wall contributes significantly to increased oscillation of the estimation error as function of the imposed IMT values (Figs. $6.11 \mathrm{~b}, 6.11 \mathrm{~d}, 6.11 \mathrm{f}, 6.11 \mathrm{~h}$ ). For an intimal echo level higher than the ringing lobe of the adventitial one, the oscillations appear for IMT values up to $500 \mu \mathrm{m}$, which are in the range of the adventitial trailing extension. When the intimal amplitude on the near wall is similar to the adventitial ringing lobe, the effects are noticeable also at higher IMT values (Fig. 6.11h). Because at the far wall the intima echo is preceding the adventitial one, such interference does not occur.

\section{References}

Baldassarre D, De Jong A, Amato M, Werba JP, Castelnuovo S, Frigerio B, Veglia F, Tremoli E, Sirtori CR. Carotid intima-media thickness and markers of inflammation, endothelial damage and hemostasis. Annals of Medicine 2008; 40: 21-44.

Brands PJ, Hoeks APG, Ledoux LAF, Reneman RS. A radio frequency domain complex cross-correlation model to estimate blood flow velocity and tissue motion by means of ultrasound. Ultrasound in Medicine \& Biology 1997; 23: 911-920.

Brands PJ, Hoeks APG, Willigers J, Willekes C, Reneman RS. An integrated system for the non-invasive assessment of vessel wall and hemodynamic properties of large arteries by means of ultrasound. European Journal of Ultrasound 1999; 9: 257-266.

Bruschi G, Cabassi A, Orlandini G, Regolisti G, Zambrelli P, Calzolari M, Borghetti A. Use of Fourier shape descriptors to improve the reproducibility of echographic measurements of arterial intima-media thickness. Journal of Hypertension 1997; 15: 467-474.

Burckhardt CB. Speckle in ultrasound B-Mode scans. IEEE Transactions On Sonics And Ultrasonics 1978; 25: 1-6.

De Groot E, Hovingh K, Wiegman A, Duriez P, Smit AJ, Fruchart JC, Kastelein JP. Measurement of arterial wall thickness as a surrogate marker for atherosclerosis. Circulation 2004; 109: 33-38.

Demi M. The first absolute central moment in low-level image processing. Computer Vision and Image Understanding 2000; 80: 57-87.

Demi M. The first absolute central moment as an edge detector. Nonlinear Analysis 2001; 47: 5185-5826. 
Dineley JA, McDicken WN, Hoskins PR. Factors affecting the arterial distension waveform derived from tissue Doppler imaging (TDI): an in vitro study on precision. Ultrasound in Medicine \& Biology 2007; 33 : 1123-1131.

Egger M, Krasinski A, Rutt BK, Fenster A, Parraga G. Comparison of B-mode ultrasound, 3-dimensional ultrasound, and magnetic resonance imaging measurements of carotid atherosclerosis. Journal of Ultrasound in Medicine 2008; 27: 1321-1334.

Ellis SM, Naomuova RP, Neuwirth CK, Eckersley R, Cosgrove DO, Thompson GR, Sidhu PS. Measurement of the reflectivity of the intima-medial layer of the common carotid artery improves the discriminatory value of intima-medial thickness measurement as a predictor of risk of atherosclerotic disease. Ultrasound in Medicine \& Biology 2007; 33: 1029-1038.

Faita F, Gemignani V, Bianchini E, Giannarelli C, Ghiadoni L, Demi M. Real-time measurement system for evaluation of the carotid intima-media thickness with a robust edge operator. Journal of Ultrasound in Medicine 2008; 27: 1353-1361.

Gemignani V, Paterni M, Benassi A, Demi M. Real time contour tracking with a new edge detector. Real Time Imaging 2004; 10: 103-116.

Gemignani V, Faita F, Ghiadoni L, Poggianti E, Demi M. A system for real-time measurement of the brachial artery diameter in B-mode ultrasound images. IEEE Transactions On Medical Imaging 2007; 26: 393 404.

Hoeks APG, Di X, Brands PJ, Reneman RS. An effective algorithm for measuring diastolic artery diameter. Archives Of Acoustics 1995; 20: 65-76.

Hoeks APG, Hermeling E, Reneman RS, Artery wall mechanics determined by means of ultrasound. In: Vascular Hemodynamics Rounds, John Wiley and Sons, New York, 2008.

Laurent S, Cockroft J, Van Bortel L, Boutouyrie P, Gianattasio C, Hayoz D, Pannier B, Charalambos V, Wilkinson I, Struijker-Boudier H. Expert consensus document on arterial stiffness: methodological issues and clinical applications. European Heart Journal 2006; 27: 2588-2605.

Lorenz MW, Markus HS, Bots ML, Rosvall M, Sitzer M. Prediction of clinical cardiovascular events with carotid intima-media thickness: a systematic review and meta-analysis. Circulation 2007; 115: 459-467.

Mallett C, House AA, Spence JD, Fenster A, Parraga G. Longitudinal ultrasound evaluation of carotid atherosclerosis in one, two and three dimensions. Ultrasound in Medicine \& Biology 2008; 35: 367-375.

Mattace-Raso FUS, Van Der Cammen TJM, Hoffman A, Van Popele NM, Bos ML, Schalekamp MADH, Asmar R, Reneman RS, Hoeks APG, Breteler MMB, Witteman JCM. Arterial stiffness and risk of coronary heart disease and stroke - The Rotterdam study. Circulation 2006; 113: 656-663.

Meinders JM, Brands PJ, Willigers JM, Kornet L, Hoeks APG. Assessment of the spatial homogeneity of artery dimension parameters with high frame rate 2-D B-mode. Ultrasound in Medicine \& Biology 2001; 27: 785-794.

Potter K, Reed CJ, Green DJ, Hankey GJ, Arnolda LF. Ultrasound settings significantly alter lumen and wall thickness measurements. Cardiovascular Ultrasound 2008; 6: DOI: 10.1186/1476-7120-6-6.

Reneman RS, Meinders JM, Hoeks APG. Non-invasive ultrasound in arterial wall dynamics in humans: what we have learned and what remains to be solved. European Heart Journal 2005; 26: 960-966.

Riley WA, Evans GW, Sharrett AR, Burke GL, Barnes RW. Variation of common carotid artery elasticity with intimal-medial thickness: the ARIC study. Ultrasound in Medicine \& Biology 1997; 23: 157-164. 
Rossi AC, Brands PJ, Hoeks APG. Fully automatic assessment of carotid artery curvature and diameter with non-invasive ultrasound. 2008a. IFMBE Proceedings - 4th European Conference of the International Federation for Medical and Biological Engineering, (Antwerp, Belgium, 23-27 November 2008). 22: 588591. Springer.

Rossi AC, Brands PJ, Hoeks APG. Automatic recognition of the common carotid artery in longitudinal ultrasound B-mode scans. Medical Image Analysis 2008b; 12: 653-665.

Rossi AC, Brands PJ, Hoeks APG. Nonlinear processing in B-mode ultrasound affects carotid diameter assessment. Ultrasound in Medicine \& Biology 2009; 35: 736-747.

Schmidt-Trucksass A, Cheng D, Sandcrock M, Sculte-Monting J, Rauramaa R, Huonker M, Burkhardt H. Computerized analyzing system using the active contour in ultrasound measurement of carotid artery intima-media thickness. Clinical Physiology 2001; 21: 561-569.

Selzer RH, Hodis HN, Kwong-Fu H, Mack WJ, Lee PL, Liu CR, Liu CH. Evaluation of computerized edge tracking for quantifying intima-media thickness of the common carotid artery from B-mode ultrasound images. Atherosclerosis 1994; 111: 1-11.

Spence JD. Measurement of intima-media thickness vs. carotid plaque: uses in patient care, genetic research and evaluation of new therapies. International Journal of Stroke 2006; 1: 216-221.

Stadler RW, Karl WC, Lees RS. New methods for arterial diameter measurement from B-mode images. Ultrasound in Medicine \& Biology 1996; 22: 25-34.

Stein JH, Korkarz CE, Mays ME, Douglas PS, Palta M, Zhang H, LeCaire T, Paine D, Gustafson D, Fan L. A semiautomated ultrasound border detection program that facilitates clinical measurement of ultrasound carotid intima-media thickness. Journal of the American Society of Echocardiography 2005; 18: 244-251.

Touboul P-J, Hennerici MG, Meairs S, Adams H, Amarenco P, Bornstein N, Csiba L, Desvarieux M, Ebrahim S, Fatar M, Hernandez Hernandez R, Jaff M, Kowantor S, Prati P, Rundek T, Sitzer M, Schminke U, Cardif JC, Taylor A, Vicaut E, Woo KS, Zannad F, Zureik M. Mannheim carotid intima-media thickness consensus (2004-2006). Cerebrovascular Diseases 2007; 23: 75-80. 

CHAPTER 7

General discussion 
This thesis presents and evaluates various computational methods for the automatic estimation of morphological and dynamic properties of the common carotid artery (CCA). The analysis is based on ultrasonic echo amplitude signals retrieved directly from radio frequency (RF) ultrasound data in ultrasound B-mode images. Despite the low frame update rate of $30 \mathrm{~Hz}$ the algorithms provide reliable waveforms for CCA diameter and intima-media thickness over long recording times without user interference and without drift as normally associated with phase-sensitive wall-tracking algorithms. Although the algorithms are developed and evaluated from an engineering/image-processing point of view, they are designed according to their aim and future applicability in a clinical framework. A direct interaction between technological development and clinical scope is typical for the field of biomedical engineering, which contextualizes the thesis.

As indicated in the introductory chapters, proper image segmentation is the key step in the estimation of CCA position, diameter and IMT from B-mode ultrasound scans. Even though manual and semi-automatic methods for segmentation of vascular ultrasound images are largely in use, they are extremely time-consuming compared to automatic methods while they are much less suited for proper standardization. Considering the large number of mechanical and dynamic parameters that can be derived from non-invasive estimates of CCA diameter and intima-media thickness (IMT), it is clear that poor precision and repeatability of such measured geometrical properties negatively affects the clinical value. Therefore, segmentation of the acquired ultrasound vascular scans has a large impact on the relevance of the outcomes and should be as objective and robust as possible, regardless of its realtime or off-line implementation. That is why in this thesis, processing is mostly based on the underlying received radio-frequency (RF) signal, retaining its amplitude on a linear scale even though images are presented on a compressed scale. This approach allows us to consider errors associated with non-linear acquisition and processing procedures as compared to linear procedures. Moreover, newly introduced contour detection methods are designed and evaluated without the interference of ultrasound visualization settings, as is instead common when processing ultrasound images digitally grabbed from screen.

Chapter 3 presents a comparative analysis of two edge detection procedures for the measurement of the external (adventitia-adventitia) diameter of the CCA, namely the sustain-attack filter (SAF) and the derivative approach, including non-linear effects. Fig 3.6 shows that logarithmic compression has a significant effect on the absolute values of the estimated diameter throughout the cardiac cycle. When using the derivative approach, the difference in diameter between linear and logcompressed versions of the same recording is in the order of $10 \%$ of the external CCA diameter. The sustain-attack filter proves to be more robust and precise, and 
deals inherently with the logarithmic compression characteristics of the ultrasound system. It might be argued that also for the derivative approach inversion of the logarithmic compression would be a suitable solution. However, logarithmic compression often integrates some kind of thresholding on the signals, which is not invertible. Thresholding obviously affects the sustain-attack filter outcome as well, but hardly affects the reference function for edge detection. Moreover, derivation is much more susceptible to image noise than the sustain-attack filter, as is demonstrated by the higher intrarecording and intrasubject variabilities (Figs. 3.7 and 3.8), both for linear and log-compressed cases. The sustain-attack filter is, therefore, the first choice for delineation of the CCA external diameter. Even though we strongly recommend analyzing uncompressed data where possible, it is still a very common approach to segment ultrasound images based on their screen appearance including saturation and log-compression. The relevance of compensating for non-linear processing characteristics of ultrasound systems has already been addressed in relation to speckle reduction (Crawford 1993; Dutt 1996), and we believe it is also of high importance for image segmentation.

It is worth noting that the observed yearly increase in CCA IMT is in the order of 5 to $10 \mu \mathrm{m}$ (Ishizu 2004; Juonala 2008), whereas external CCA diameter increases about $10 \mu \mathrm{m}$ per year (Samijo 1998), which is slightly higher than the IMT increase. Of course, these values are statistically deduced from large population studies, as they are below the resolution of common ultrasound systems (in the order of 200-300 $\mu \mathrm{m})$. These values are also below the errors in diameter estimation (Chapter 3 ) due to saturation (100-200 $\mu \mathrm{m})$ and compression $(600 \mu \mathrm{m})$. Accounting for saturation and log-compression would improve measurement precision, and would allow population studies with a substantially lower number of subjects for follow-up (e.g. a 2-fold decrease in standard deviation of the estimates would imply a 4-fold decrease in the number of subjects to reach the significance level).

The heterogeneity of ultrasound protocols is probably one of the major determinants of the variability found in the relationships between IMT and risk (Lorenz 2007). The variability may limit the ability of IMT-based studies to predict clinical cardiovascular end points. According to the standard protocol, IMT analysis should be performed on a plaque-free CCA segment (Touboul 2007), which explains the focus of this thesis on the CCA (as opposed to the bulb, where plaques are more likely to form). One of the main operational nuisances for a sonographer is the task to manually indicate the whereabouts of the artery prior to the segmentation procedure. This generally has to be done at the start of each measurement, and repeated whenever the artery leaves the image plane due to subject or probe motion. That is why in Chapter 4 we introduce a novel on-line algorithm for the automatic recognition of the CCA in longitudinal ultrasound B-mode scans. We developed the 
method taking into consideration the drawbacks of previous attempts (Fan 2000; Delsanto 2007). Our method is based on decimation, parametrical template matching and clustering, and is effective with a hit rate exceeding $98 \%$ in 128 ultrasound data sets of 6 seconds each, for a total of 6185 processed frames. From an image processing point of view, it is quite a challenge to develop such robust algorithm because it is widely known that automatic recognition (i.e. the association of a semantic meaning) of a structure in an image is a much more high level task than its delineation (Udupa 2006). The signal-processing concept allows real-time implementation, as recently demonstrated by Esaote Europe (Maastricht, the Netherlands) by translating the MATLAB code to a $\mathrm{C}++$ implementation. The principal bottleneck for fully automated processing of CCA ultrasound scans is thus eliminated. In its off-line implementation, as described in Chapter 4, the automatic CCA recognition algorithm is also very useful to analyze huge amounts of ultrasound frames without the need for human supervision, as shown by its subsequent incorporation in the CCA segmentation methods described in Chapters 5 and 6. Presently the application of the method is restricted to ultrasound frames showing only a single vessel, the CCA. The presence of the jugular vein in the image can occasionally mislead the CCA recognition algorithm. We decided not to tackle this issue for the moment because clinical reports demonstrate that CCA scanning with the jugular vein upfront quite likely induces reverberation and mirroring artifacts (Kurtz 2008) that can complicate CCA analysis, and at times may even erroneously suggest carotid dissections.

Chapter 5 introduces a method for the automatic estimation of the CCA orientation and curvature on a frame-by-frame basis. The algorithm also takes advantage of the CCA centerline delineation in order to re-align the frame horizontally and subsequently to perform lateral median filtering independent of the original orientation of the artery. The filtering procedure reduces speckle artifacts without decreasing the vertical resolution perpendicularly to arterial walls. Speckle reduction techniques are commonly based on smoothing images in both directions (Michailovich 2006), thereby degrading contour delineation. Our method, used to delineate adventitial walls by means of sustain-attack filtering, can be seen as a simple and pragmatic alternative to more complex directional filtering techniques (Guo 2009). Fig. 5.4 shows that the lateral median filtering improves the precision of external CCA diameter estimates. Furthermore, the possibility to track orientation and curvature along with the diameter waveform (Fig. 5.3) allows to compensate diameter (and IMT, see Chapter 6) estimates for deviations from perpendicularity. Doppler ultrasound, which strongly relies on the inclined angle between observation and flow velocity direction, would also benefit from line-by-line orientation estimation. 
In Chapter 6 we propose a CCA wall delineation method for the intimal layer of both walls. The multiscale anisotropic barycenter (MAB), which we developed based on the "first order absolute central moment" (Gemignani 2007), strongly deviates from the sustain-attack filter concept for the adventitia. The combined algorithm delineates intima and adventitial layers on both walls of the common carotid artery, yielding estimates of near wall IMT, far wall IMT, internal diameter and external diameter. Tracking such quantities simultaneously in time, as shown in Fig. 6.5, is important in order to understand differences in relative distension of the CCA inner and outer walls (Segers 2004). It is also of importance to evaluate the interrelations between diameter and IMT during the cardiac cycle or, on a wider time scale, to investigate remodeling processes (Glagov 1995; Bots 2005). Those issues are relevant to differentiate the effects of age and atherosclerosis on adventitial diameter, luminal diameter, wall thickness and elasticity (Reneman 1986; Labropoulos 1998; Ishizu 2004; Jurasic 2007; Juonala 2008).

The multiscale anisotropic barycenter approach, relying on the previously applied sustain-attack filter, follows an iterative implementation using anisotropic concentric kernels, for smoothing and evaluation, and dedicated post processing. The method can handle ultrasound images prior to scan-conversion and, hence, is compatible with anisotropic sampling. The "multiscale" term refers to the fact that at each iteration the filtering kernel resizes according to the distance from its centre to the adventitial border. The algorithm has the peculiarity to delineate an intimal layer only where it is actually "detectable". No spatial or temporal smoothing is used to regularize the appearance of the intimal overlay, leaving gaps in the contours (see Fig. 6.4 for instance). Because the contours are locally interrupted, the local echogenicity of the intima with respect to that of the blood remains visible. For the sonographer, those gaps are very informative, in line with the fact that ultrasound imaging strongly relies on a direct feedback. In future real-time implementations of the multiscale anisotropic barycenter method, the contour gaps should prompt the sonographer to reorient the plane of view, and to measure IMT in a CCA segment having as less gaps as possible. A similar gap feature is already implemented in the ART.LAB system (Esaote Europe, Maastricht, The Netherlands) (Brands 1999), used to measure morphological and dynamic arterial characteristics based on RF data sets.

Wall delineation algorithms with smoothing (intended as intrinsic gap-filling) by means of active contours (Schmidt-Trucksass 2001; Cheng 2002; Delsanto 2007) introduce two possible confounding factors: the heterogeneous echogenicity of the intimal layer is hidden behind the contour, and the contour position is averaged also in zones where its value is not directly measured, but inferred from the surroundings. Of course, this will yield diameter and IMT waveforms that are looking smooth, 
but the smoothness achieved in such way is not directly related to the quality of the recording and may create falsely the illusion of an accurate measurement. The contour obtained within each acquisition is rather arbitrary and dictated by the active contour properties, i.e. rigidity and temporal inertia. We recommend an artery delineation approach that retains possible irregularities of the intima-media complex. It has recently been shown that wall irregularity is associated with nearby atherosclerosis (Graf 2009). In Chapter 5 we do include directional smoothing as a pre-processing step, but only for the media-adventitia transition using SAF.

The combination of sustain-attack filter and multiscale anisotropic barycenter, as introduced in Chapter 6, provides contours that are suitable to grade insonation quality. The consistency of the insonation plane is crucial for diameter and IMT assessment (Stadler 1996; Bruschi 1997), mainly because the ultrasound backscatter amplitude highly depends on the angle between the ultrasonic beam and the structure being imaged (De Kroon 1991). That is why we integrated an automatic scanplane control, based on geometrical constraints as well as the relative amount of gaps in each contour. Visualization of such information by means of color encoding would eventually help sonographers to make their "learning curve" faster regarding B-mode arterial imaging. Because user experience affects inter-observer concordance (Haluska 2001), scan plane control can help to attain a better standardization of ultrasound protocols. For instance, in a future real-time implementation the sonographer, assisted by the automatic algorithm we proposed in Chapter 4 to localize an artery and visually informed about the insonation plane incidence by the color of intima and adventitia overlays, would be allowed to focus on proper probe positioning.

The CCA delineation method of Chapter 6 also adjusts contour positions according to the relative echo amplitudes of intima and adventitia (Figs 6.3d and 6.3e) to correct for differences between the contour algorithm for the adventitia (sustainattack filter: SAF) and intima (multiscale anisotropic barycenter: MAB). Such equalization of intimal and adventitial positions is only possible for images without saturation, which is also relevant for clinical studies. It has been demonstrated in relatively small patient groups that CCA echo intensity is related to myocardial infarction (Takiuchi 2000) and wall properties (Kawasaki 2005). Referring to relative echo amplitudes can improve the robustness of IMT estimation in relation to wall reflectivity (Ellis 2007). The effectiveness of our proposed correction for relative echo amplitudes in contour detection is demonstrated by the accuracy analysis performed on synthetic images (Fig. 6.6, Table 6.1, Fig. 6.11). IMT estimates are more accurate when the adventitial and intimal contour points are placed at similar relative echo levels, even though those contours points are obtained with different edge detection algorithms. The synthetic image analysis also reveals that the echo 
amplitude ratio of adventitia and intima affects IMT estimates differently for near and far CCA walls (see Appendix 6b). A relatively lower intima echo amplitude does not affect far wall estimates, whereas the same decrease for the near wall yields estimates with poorer accuracy and precision (Fig. 6.11). The degradation is explained by phase interference between the adventitial trailing lobe and the intima echo for a closely spaced intima and adventitia. This supports the notion that near wall and far wall IMT estimates should be considered and retained separately, not averaged (Touboul 2007). However, clinical studies on the prediction of cardiovascular events with carotid IMT still occasionally combine near and far wall IMT values (Lorenz 2007). We favor the use of far wall IMT only because it is less susceptible for ultrasound-related artifacts.

The algorithm described in Chapter 6 is both accurate and precise, showing intrarecording beat-to-beat variations on average lower than $50 \mu \mathrm{m}$ for IMT and external diameter, and lower than $100 \mu \mathrm{m}$ for internal diameter. As shown in Figs. 3.6, 5.3 and 6.5, the frame-by-frame delineation results in realistic CCA diameter waveforms even for a low frame rate ( $30 \mathrm{~Hz}$ in B-mode). The low frame rate precludes further smoothing of the waveforms. However, current ultrasound scanners can reach frame rates in the order of $100-200 \mathrm{~Hz}$, which would permit regularization of diameter waveforms by smoothing. A proper visualization of diameter and IMT waveforms in real-time, next to the segmentation overlay on the ultrasound image, would also improve the sonographer's confidence in CCA scan quality. The latter, as shown in Fig. 6.5, could be indicated by the color of the waveforms. The dynamics of diameter and IMT waveforms in combination with an active scan-plane control would help ascertain that observed circumferential and radial strain patterns are due to arterial mechanics (Carallo 1999; Paini 2007), rather than to movements of an artery relative to the ultrasonic beam.

The nature of diameter waveforms as obtained in Chapters 3, 5 and 6 from B-mode images differs fundamentally from that of waveforms obtained with the wall track algorithm. The latter procedure is based on the phase behavior of RF-signals originating from the adventitia over time and readjusts dynamically the measurement volume to the observed displacement. This carries inherently the risk that errors will accumulate and the displacement waveform will exhibit drift (Brands 1999). Because of this complication, the wall track program is either restricted to a recording duration of maximally 5-6 seconds (respiration cycle) or is reset at each cardiac cycle. Displacement detection based on the B-mode image, as proposed in this thesis, does not suffer from drift as each subsequent image is treated independently. For long-term recordings, e.g. for baroreceptor sensitivity studies, it would be advantageous to incorporate a high frame rate B-mode tracking protocol. 
In the present study we do not consider plaques, but in a further development the multiscale anisotropic barycenter approach and its scan-plane control should also be able to cope with plaque analysis. Another future development could involve the application of the algorithm described in Chapter 4 to images containing more than one vessel.

The automatic techniques described in this thesis are diverse in terms of both signal processing and segmentation tasks. The work is driven by the notion that acquisition and processing methods should be standardized to fully exploit the potential of ultrasound imaging for the prevention of cardiovascular diseases in clinical practice (De Groot 2004). Moreover, they should be easy to use, for screening and in population studies, to properly address questions regarding interrelationships between carotid wall thickness, diameter and, ultimately, stiffness (Benetos 2002; Boutouyrie 2008) and pressure (Meinders 2004; Laurent 2006; Wizner 2009). Relating morphological and dynamic properties of the CCA, measured non-invasively with ultrasound, to cardiovascular disease remains difficult because of the confounding influence of aging and pressure (hypertension) on arterial stiffness (Nichols 1998). The complexity should not be further aggravated by artifacts related to ultrasound image processing and presentation, which impedes comparison of results from different clinical studies (Lorenz 2007). To harmonize the outcomes of ultrasonic clinical trials, CCA segmentation should be based on analysis of signals retaining linear amplitude, followed by segmentation algorithms with improved visual feedback to the sonographer. Automation of routine scans (artery positioning, orientation, delineation, and scan plane control) will help ultrasound operators to improve their experience and to fully concentrate on image acquisition, rather than on distracting, tedious and unnecessary tasks. The robustness and clinical relevance of non-invasive vascular ultrasound would therefore benefit from the computational methods presented in this thesis.

\section{References}

Benetos A, Waeber B, Izzo J, Mitchell G, Resnick L, Asmar R, Safar M. Influence of age, risk factors, and cardiovascular and renal disease on arterial stiffness: clinical applications. American Journal of Hypertension 2002; 15: 1101-1108.

Bots ML, Grobee DE, Hofman A, Witteman JCM. Common carotid artery intima-media thickness and risk of acute myocardial infarction. The role of lumen diameter. Stroke 2005; 36: 762-767.

Boutouyrie P, Laurent S, Briet M. Importance of arterial stiffness as cardiovascular risk factor for future developments of new types of drugs. Fundamental \& Clinical Pharmacology 2008; 22: 241-246. 
Brands PJ, Hoeks APG, Willigers J, Willekes C, Reneman RS. An integrated system for the non-invasive assessment of vessel wall and hemodynamic properties of large arteries by means of ultrasound. European Journal of Ultrasound 1999; 9: 257-266.

Bruschi G, Cabassi A, Orlandini G, Regolisti G, Zambrelli P, Calzolari M, Borghetti A. Use of Fourier shape descriptors to improve the reproducibility of echographic measurements of arterial intima-media thickness. Journal of Hypertension 1997; 15: 467-474.

Carallo C, Irace C, Pujia A, De Franceschi MS, Crescenzo A, Motti C, Cortese C, Mattioli PL, Gnasso A. Evaluation of common carotid hemodynamic forces: relations with wall thickening. Hypertension 1999; 34: 217-221.

Cheng D, Schmidt-Trucksass A, Cheng K, Burkhardt H. Using snakes to detect the intimal and adventitial layers of the common carotid artery wall in sonographic images. Computer Methods and Programs in Biomedicine 2002; 67: 27-37.

Crawford DC, Bel DS, Bamber JC. Compensation for the signal processing characteristics of ultrasound Bmode scanners in adaptive speckle reduction. Ultrasound in Medicine \& Biology 1993; 19: 469-485.

De Groot E, Hovingh K, Wiegman A, Duriez P, Smit AJ, Fruchart JC, Kastelein JP. Measurement of arterial wall thickness as a surrogate marker for atherosclerosis. Circulation 2004; 109: 33-38.

De Kroon MGM, Van Der Wal LF, Gussenoven WJ, Bom N. Angle-dependent backscatter from the arterial wall. Ultrasound in Medicine \& Biology 1991; 17: 121-126.

Delsanto S, Molinari F, Giusetto P, Liboni W, Badalamenti S, Suri JS. Characterization of a completely user-independent algorithm for carotid artery segmentation in 2-D ultrasound images. IEEE Transactions On Instrumentation And Measurement 2007; 56: 1265-1274.

Dutt V, Greenleaf JF. Adaptive speckle reduction filter for log-compressed B-Scan images. IEEE Transactions On Medical Imaging 1996; 15: 802-813.

Ellis SM, Naomuova RP, Neuwirth CK, Eckersley R, Cosgrove DO, Thompson GR, Sidhu PS. Measurement of the reflectivity of the intima-medial layer of the common carotid artery improves the discriminatory value of intima-medial thickness measurement as a predictor of risk of atherosclerotic disease. Ultrasound in Medicine \& Biology 2007; 33: 1029-1038.

Fan L, Santiago P, Jiang H, Herrington DM. Ultrasound measurement of brachial flow-mediated vasodilator response. IEEE Transactions On Medical Imaging 2000; 19: 621-631.

Gemignani V, Faita F, Ghiadoni L, Poggianti E, Demi M. A system for real-time measurement of the brachial artery diameter in B-mode ultrasound images. IEEE Transactions On Medical Imaging 2007; 26: 393 404.

Glagov S, Bassiouny HS, Giddens DP, Zarins CK. Intimal thickening: morphogenesis, functional significance and detection. Journal of Vascular Investigation 1995; 1: 2-14.

Graf IM, Schreuder FHBM, Hameleers JM, Mess WH, Reneman RS, Hoeks APG. Wall irregularity rather than intima-media thickness is associated with nearby atherosclerosis. Ultrasound in Medicine \& Biology 2009; DOI: 10.1016/j.ultrasmedbio.2008.12.016.

Guo Y, Cheng HD, Tian J, Zhang Y. A novel approach to speckle reduction in ultrasound imaging. Ultrasound in Medicine \& Biology 2009; 35: 628-640. 
Haluska B, Sutherland A, Case C, Kennedy R, Marwick TH. Automated edge-detection technique for measurement of brachial artery reactivity: a comparison of concordance with manual measurements. Ultrasound in Medicine \& Biology 2001; 27: 1285-1289.

Ishizu T, Ishimitzu T, Yanagi H, Seo Y, Obara K, Moriyama N, Shigeiuki W, Yamaguchi I. Effect of age on carotid arterial intima-media thickness in childhood. Heart Vessels 2004; 19: 189-195.

Juonala $M$, Kähönen $M$, Laitinen $T$, Hutri-Kähönen $N$, Jokinen $E$, Taittonen L, Pietikäinen $M$, Helenius $H$, Viikari JSA, Raitakari OT. Effect of age and sex on carotid intima-media thickness, elasticity and brachial endothelial function in healthy adults: the Cardiovascular Risk in Young Finns Study. European Heart Journal 2008; 29: 1198-1206.

Jurasic M-J, Lovrencic-Huzjan A, Bedekovich MR, Demarin V. How to monitor vascular aging with ultrasound. Journal of the Neurological Sciences 2007; 257: 139-142.

Kawasaki M, Ito Y, Yokoyama H, Arai M, Takemura G, Hara A, Ichiki Y, Takatsu H, Minatoguchi S, Fujiwara $\mathrm{H}$. Assessment of arterial medial characteristics in human carotid arteries using integrated backscatter ultrasound and its histological implications. Atherosclerosis 2005; 180: 145-154.

Kurtz A. Mirror-image artifact mimicking a carotid artery dissection. Journal of Diagnostic Medical Sonograohy 2008; 24: 26-29.

Labropoulos N, Zarge J, Mansour A, Kang SS, Baker WH. Compensatory arterial enlargement is a common pathobiologic response in early atherosclerosis. The American Journal Of Surgery 1998; 176: 140-143.

Laurent S, Tropeano Al, Boutouyrie P. Pulse pressure reduction and cardiovascular protection. Journal of Hypertension (Supplement) 2006; 24: S13-S18.

Lorenz MW, Markus HS, Bots ML, Rosvall M, Sitzer M. Prediction of clinical cardiovascular events with carotid intima-media thickness: a systematic review and meta-analysis. Circulation 2007; 115: 459-467.

Meinders JM, Hoeks APG. Simultaneous assessment of diameter and pressure waveforms in the carotid artery. Ultrasound in Medicine \& Biology 2004; 30: 147-154.

Michailovich OV, Tannenbaum A. Despeckeling of medical ultrasound images. IEEE Transactions On Ultrasonics, Ferroelectrics and Frequency Control 2006; 53: 64-78.

Nichols WW, O'Rourke MF. McDonald's blood flow in arteries. Theoretical, experimental and clinical principles. Arnold, 1998.

Paini A, Boutouyrie P, Calvet D, Zidi M, Agbiti-Rosei E, Laurent S. Multiaxial mechanical characteristics of carotid plaque. Analysis by multiarray echotracking system. Stroke 2007; 38: 117-123.

Reneman RS, Van Merode T, Hick P, Muytjens A, Hoeks APG. Age-related changes in carotid arterial wall properties in men. Ultrasound in Medicine \& Biology 1986; 12: 465-471.

Samijo SK, Willingers JM, Barkhuysen R, Kitslaar PJEHM, Reneman RS, Brands PJ, Hoeks APG. Wall shear stress in the human common carotid artery as function of age and gender. Cardiovascular Research 1998; 39: 515-522.

Schmidt-Trucksass A, Cheng D, Sandcrock M, Sculte-Monting J, Rauramaa R, Huonker M, Burkhardt H. Computerized analyzing system using the active contour in ultrasound measurement of carotid artery intima-media thickness. Clinical Physiology 2001; 21: 561-569. 
Segers P, Rabben SI, De Backer J, De Sutter J, Gillebert TC, Van Bortel L, Verdonck P. Functional analysis of the common carotid artery: relative distension differences over the vessel wall measured in vivo. Journal of Hypertension 2004; 22: 973-981.

Stadler RW, Karl WC, Lees RS. New methods for arterial diameter measurement from B-mode images. Ultrasound in Medicine \& Biology 1996; 22: 25-34.

Takiuchi S, Rakugi H, Honda K, Masuyama T, Hirata N, Ito H, Sugimoto K, Yanagitani Y, Moriguchi K, Okamura A, Higaki J, Ogihara T. Quantitative ultrasonic tissue characterization can identify high-risk atherosclerotic alteration in human carotid arteries. Circulation 2000; 102: 766-770.

Touboul P-J, Hennerici MG, Meairs S, Adams H, Amarenco P, Bornstein N, Csiba L, Desvarieux M, Ebrahim S, Fatar M, Hernandez Hernandez R, Jaff M, Kowantor S, Prati P, Rundek T, Sitzer M, Schminke U, Cardif JC, Taylor A, Vicaut E, Woo KS, Zannad F, Zureik M. Mannheim carotid intima-media thickness consensus (2004-2006). Cerebrovascular Diseases 2007; 23: 75-80.

Udupa JK, LeBlanc VR, Zhuge Z, Imieliska C, Schmidt H, Currie LM, Hirsch BE, Woodburn J. A framework for evaluating image segmentation algorithms. Computerized Medical Imaging And Graphics 2006; 30: 75-87.

Wizner B, Dechering DG, Thijs L, Kuznetsova T, Richart T, Jin Y, Gasowski J, O'Brien E, Struijker-Boudier HA, Grodzicki T, Staessen JA. Conventional and 24-h ambulatory blood pressure as independent predictors of elastic arterial properties. Blood Pressure Monitoring 2009; 14: 12-19. 



\section{Summary}

Non-invasive estimates of common carotid artery (CCA) anatomical features by means of ultrasound (US), including diameter and intima-media thickness (IMT), are gaining importance for estimation of arterial mechanical properties in relation to prevention and treatment of cardiovascular diseases, and assessment of cardiovascular risk. Many different image processing methods are nowadays available for assessing CCA diameter and IMT on 2-dimensional ultrasonic images, usually based on the grey level gradient or on active contours. Those methods present common drawbacks related to user interference and to pre-processing of the ultrasound images for visual optimization.

In this thesis we introduce new computational methods for processing of longitudinal B-mode US scans of the CCA. They are based on the direct analysis of ultrasound radio-frequency signals, for the automatic estimation of the time-dependent CCA position, CCA orientation, CCA internal and external diameter, and CCA IMT of both near and far arterial walls. Echo-images show an artery as an area with a low signal level (lumen) bounded by a structure producing a double line pattern. The inner line is generated by the lumen-intima transition, followed by a region with a relatively low echo-level (the media) and a region with a rather high amplitude (the adventitia and surrounding tissue). We focused on implementing algorithms that can provide sonographers an improved feedback during ultrasound B-mode scans.

Chapter 1 presents a general introduction about cardiovascular disease, and noninvasive estimation of arterial properties by means of ultrasound. Chapter 2 introduces basic concepts of ultrasound physics and technology.

An in-depth analysis of the effects of non-linear ultrasound image presentation (logarithmic compression and signal saturation) on automatic CCA external diameter estimation is provided in Chapter 3. Two different edge detection methods are compared on 41 acquisitions from 14 healthy subjects: sustain-attack filter and derivative approach. Diameter values assessed with the sustain-attack filter are unaffected by logarithmic compression. The estimated diameters values obtained with the derivative approach instead show differences in the order of $10 \%$ due to compression. Also saturation affects the derivative more than the sustain-attack filter. Therefore the sustain-attack filter is more suited for vascular ultrasound image segmentation algorithms. 
Chapter 4 introduces a novel algorithm for the automatic recognition of the CCA in ultrasound images, based on decimation, template matching and clustering. It allows fully automatic arterial segmentation without user interference. The processing parameters are tuned via off-line testing on 128 echo data recordings from 45 subjects. Correct and fast recognition of the artery is achieved in more than $98 \%$ of the 6185 processed frames, proving that the proposed automatic algorithm is suitable for real-time recognition of the CCA.

Chapter 5 describes a method, based on the sustain-attack filter, for the estimation of local orientation and curvature along the CCA centerline, in combination with a directional smoothing of the CCA walls for better precision in external diameter estimation. The method is tested on 30 US recordings from 10 healthy volunteers. The algorithm successfully detects CCA orientation, curvature and diameter for every subject considered. The standard deviation of wall position estimates decreases significantly when the proposed pre-processing is applied. Moreover, it allows correction of the diameter values based on local orientation.

Chapter 6 introduces an automatic methodology for the combined estimation of internal and external CCA diameter waveforms, as well as IMT waveforms of both carotid walls. The algorithm, based on the sustain-attack filter and on the multiscale anisotropic barycenter (MAB), iteratively searches for the lumen-intima transition within a small region of interest. An automatic measure of the quality of the US beam incidence for each wall is superimposed on the CCA contour overlays for visual feedback. Validation is carried out on 36 acquisitions from 12 healthy volunteers, as well as on synthetic images. Results indicate good accuracy and precision, with Intra-recording beat-to-beat variations on average lower than $50 \mu \mathrm{m}$ for external diameter and IMT, and lower than $100 \mu \mathrm{m}$ for internal diameter. Improved precision of the estimates is relevant for a parameter such as IMT, which has a small annual increase of less than $10 \mu \mathrm{m} / \mathrm{y}$. The beam incidence control significantly increases the repeatability of IMT estimates, and is intended to actively motivate sonographers to maintain a proper scan plane throughout the acquisition. This minimizes the incidence of confounding factors in non-invasive CCA ultrasonic examinations.

Discussion and conclusion of the thesis are presented in Chapter 7. We conclude that the automatic algorithms introduced effectively reduce the variability of the estimates, while compensating for typical nuisances of ultrasound imaging due to speckle and presentation strategies. This will facilitate comparison of results from different clinical studies in large clinical trials, and permit population studies to reach clinical relevance with a lower number of recruited subjects. The methods introduced in this thesis allow further standardization of ultrasound protocols for 
the assessment of CCA morphological and dynamic properties. Automation of routine scans (artery position, orientation, delineation, and scan plane control) gives improved feedback to sonographers, allowing them to fully concentrate on image acquisition and increasing the robustness of outcomes in non-invasive vascular ultrasound. 


\section{Samenvatting}

De afmetingen van de halsslagader, zoals de lumen diameter en wanddikte, spelen een belangrijke rol bij de bepaling van de mechanische eigenschappen van de slagaderwand met niet-invasieve ultrageluidstechnieken. Die mechanische eigenschappen zijn van belang voor de preventie en behandeling van vasculaire afwijkingen en de inschatting van het vasculaire risico. In het verleden zijn er veel verschillende technieken voorgesteld om uit de ultrageluid echobeelden de diameter en wanddikte van de halsslagader te schatten. Gewoonlijk evalueren deze technieken de grijswaarden gradiënt om uit de twee-dimensionale afbeelding, eventueel in combinatie met voorwaarden aan de vorm van de contour, de positie van de wand af te leiden. Al deze technieken hebben als gemeenschappelijke problemen dat ze gebruikersinteractie vereisen en dat het resultaat afhankelijk is van de wijze waarop de afbeelding wordt gemaakt en gepresenteerd.

Het echobeeld van de halsslagader wordt doorgaans in de lengterichting gemaakt. De structuur van slagaders komt in dit echobeeld naar voren als een gebied met een zeer lage intensiteit (het lumen), dat begrensd wordt door een 2-lijns structuur. De eerste lijn komt van de lumen-intima overgang, gevolgd door een smal gebied met een relatief lagere echo-intensiteit (de media) en een gebied met een aanzienlijk hogere signaal amplitude (de adventitia en het weefsel waarin de slagader is ingebed). In dit proefschrift introduceren we nieuwe methoden om uit het ruwe onderliggende echo-signaal de positie, oriëntatie, interne en externe diameter en de wanddikte van de halsslagader af te leiden. Bij de ontwikkeling van de analyse methoden hebben we de nadruk gelegd op die technieken die echo-laboranten gerichte feedback bieden tijdens het maken van een echo-registratie.

Hoofdstuk 1 bevat een algemene introductie over cardiovasculaire ziekten en de beschikbare ultrageluidstechnieken om de vaatwandeigenschappen niet-invasief te bepalen. Hoofdstuk 2 geeft de fysische achtergrond van de gebruikte ultrageluidstechnieken.

In Hoofdstuk 3 worden de effecten van signaal verzadiging (oversturing) en signaal compressie (logaritmische versterking) op de bepaling van de externe diameter van de halsslagader bekeken. Daartoe worden 41 registraties van 14 gezonde vrijwilligers geanalyseerd met 2 contourdetectie technieken. De ene techniek gaat uit van een lokaal maximum (de adventitia) om een drempel te vinden voor de mediaadventitia overgang. De andere techniek kijkt juist naar de gradiënt van de echoin- 
tensiteit kijkt zoals deze optreedt bij de overgang van de media naar de adventitia. Voor de evaluatie is de compressie en de mate van oversturing achteraf gevarieerd. Het blijkt dat contourdetectie uitgaande van het lokale maximum niet beïnvloed wordt door compressie, maar de gradiëntmethode vertoont diameterafwijkingen in de orde van $10 \%$. De gradiëntmethode is ook gevoeliger voor signaaloversturing en is daarom minder geschikt voor segmentatie van ultrageluidsbeelden.

In Hoofdstuk 4 wordt een nieuwe methode besproken om de halsslagader automatisch in echobeelden herkennen door achtereenvolgens de beeldinformatie te decimeren, met een sjabloon naar een lumen-wand-overgang te zoeken en de samenhang tussen de gevonden posities (clusteren) na te gaan. Met deze benadering is het mogelijk om zonder gebruikersinteractie de aanwezigheid, positie en oriëntatie van de halsslagader in een echobeeld vast te stellen. De instellingen voor de signaalverwerking zijn verkregen door 128 echoregistraties ( 6 seconden) van 45 personen te analyseren. De halslagader werd in $98 \%$ van de 6185 echobeelden herkend, wat demonstreert dat het voorgestelde algoritme bruikbaar is voor automatische, real-time, herkenning van de halsslagader.

In Hoofdstuk 5 wordt dieper ingegaan op de methode om automatisch de lokale oriëntatie en de kromming van de halsslagader uit het echobeeld af te leiden. De middeling van de wandposities overeenkomstig de gevonden lokale oriëntatie verbetert de precisie van de schatting van de externe wandpositie. Deze methode is uitgetest op 30 echoregistraties van 10 gezonde vrijwilligers. In alle gevallen bleek het algoritme in staat te zijn om zonder gebruikersinteractie, dus volledig automatisch, de positie, oriëntatie, kromming en externe diameter van de halsslagader te bepalen. De voorgestelde methode leidt tot een aanzienlijk lagere standaarddeviatie van de geschatte wandposities. Bovendien kan de waargenomen diameter gecorrigeerd worden voor de lokale oriëntatie.

In Hoofdstuk 6 introduceren we een automatische schattingsmethode van de golfvorm van zowel de interne als externe diameter van de halslagader over de hartcyclus, uitgaande van verandering in wanddikte van de voor- en achterwand als functie van de tijd. De analyse combineert de bepaling van de externe wandpositie uitgaande van het lokale maximum van het echo-signaal (hoofdstuk 3: mediaadventitia overgang) met een iteratieve zoekprocedure naar de positie van de lumen-intima overgang. Het verschil tussen de externe en interne wandpositie is dan de lokale instantane wanddikte. De kwaliteit van de echoregistratie, die in hoge mate afhankelijk is van oriëntatie van de slagader in het echobeeld en gebaseerd is op de geïdentificeerde wanddikte, wordt in kleur gecodeerd in het echobeeld meegegeven. We hebben de methode experimenteel toegepast op 36 registraties van 12 gezonde vrijwilligers en op gesimuleerde data. De precisie voor de 4-6 hartslagen 
in de registratie blijkt beter te zijn dan $50 \mu \mathrm{m}$ voor de externe diameter en beter dan $100 \mu \mathrm{m}$ voor de interne diameter. De verbeterde precisie is met name van belang voor een parameter als de wanddikte, die normaal een jaarlijkse toename heeft van minder dan $10 \mu \mathrm{m} / \mathrm{jaar}$. De kwaliteitsindicatie van de echoregistratie heeft een gunstige invloed op de betrouwbaarheid van de wanddikteschatting. Het zal de echo-laboranten ook aanmoedigen om gedurende de registratie hetzelfde scanvlak te handhaven.

Hoofdstuk 7 bevat een algemene discussie van de behaalde resultaten en conclusie. De ontwikkelde automatische analysemethoden leiden daadwerkelijk tot een lagere variatie van de schattingen doordat ze de registratie-afhankelijke effecten, zoals speckle en niet-lineare beeldbewerking, onderdrukken. De lagere standaarddeviatie van de schatting in combinatie met het gestandaardiseerde analyseprotocol maakt onderlinge uitwisseling van resultaten tussen samenwerkende instituten mogelijk en reduceert ook het aantal vereiste deelnemers voor klinische testen. De automatisering van routinematige scans (herkenning slagader, contourdetectie, oriëntatie, kromming en kwaliteitscontrole) verbetert tevens de terugkoppeling naar de echolaboranten. Daardoor kunnen ze zich volledig concentreren op de kwaliteit van de beeldregistratie. Dat verbetert de precisie van de schattingen en dus ook de resultaten van vasculair ultrageluidsonderzoek. 


\section{Sommario}

La stima non invasiva tramite ultrasonografia dei parametri anatomici della carotide comune, quali diametro dell'arteria e spessore del complesso intima-media ("intima-media thickness" o "IMT"), sta acquisendo crescente importanza per la misura delle propietà meccaniche arteriali, in relazione a prevenzione e cura di problemi cardiovascolari e stima dei fattori di rischio ad essi correlati. Sono oggigiorno disponibili vari metodi di analisi d'immagine per le misure di diametro ed IMT in scan ultrasonografici bidimensionali, usualmente basati sul gradiente dei livelli di grigio oppure su contorni attivi. Tali metodi presentano generalmente svantaggi legati alla necessità di inizializzazione manuale ed al pre-processing delle immagini ad ultrasuoni, necessario per una visualizzazione ottimale.

In questa tesi di dottorato vengono presentati nuovi metodi computazionali, applicati a sezioni longitudinali in B-mode della carotide comune e basati sull'analisi diretta di segnali ultrasonografici a radio frequenza, per la stima automatica delle dinamiche temporali della carotide comune in termini di posizione, orientamento, diametro interno ed esterno, ed IMT su entrambi i lati. Immagini ecografiche longitudinali mostrano l'arteria come un'area a bassa ecogenicità (il lume) delimitata superiormente ed inferiormente da echi aventi una struttura "a doppia linea". La linea interna è generata dalla transizione lume-intima, ed è seguita da una regione ad ecogenicità relativamente bassa (tunica media), e da un eco avente ampiezza elevata (tunica avventizia e tessuti circostanti). Gli algoritmi sono stati implementati in modo da poter fornire un feedback migliorato agli operatori durante le acquisizioni ultrasonografiche.

II Capitolo 1 consiste in un'introduzione generale sui problemi cardiovascolari, e sulla stima non invasiva delle proprietà arteriali tramite ultrasuoni. II Capitolo 2 introduce concetti di base riguardanti la fisica e la tecnologia dell'imaging ultrasonografico.

Un'analisi approfondita degli effetti che la presentazione non lineare di immagini ecografiche (compressione logaritmica e saturazione) ha sulla stima automatica del diametro esterno della carotide comune è fornita nel Capitolo 3. Due differenti metodi per la detezione di contorni vengono utilizzati in 41 acquisizioni (ognuna delle quali contenente numerose immagini ecografiche) effettuate su 14 soggetti senza problemi cardiovascolari. Gli algoritmi utilizzati analizzano gli inviluppi dei segnali a radio frequenza, e sono il cosiddetto "filtro sustain-attack" ed un approccio 
basato sulla derivata locale. I valori di diametro ottenuti con il filtro sustain-attack non sono influenzati dalla compressione logaritmica. I valori ottenuti con l'approccio derivativo invece mostrano differenze nell'ordine del $10 \%$ dovute alla compressione. Anche la saturazione influenza l'approccio derivativo maggiormente rispetto al filtro sustain-attack. Quest'ultimo è quindi più adatto per algoritmi di segmentazione di immagini ultrasonografiche vascolari.

Il Capitolo 4 presenta un nuovo algoritmo per il riconoscimento automatico della carotide comune in immagini ad ultrasuoni, basato su decimazione, template matching e clustering. II metodo permette di implementare una segmentazione dell'arteria completamente automatica senza necessità di inizializzazione manuale. I valori ottimali dei parametri dell'algoritmo sono ottenuti tramite l'analisi off-line di 128 acquisizioni ultrasonografiche effettuate su 45 pazienti. Un veloce e corretto riconoscimento automatico della carotide comune avviene in più del $98 \%$ delle 6185 immagini analizzate, e ciò dimostra come l'algoritmo sia adatto per un riconoscimento in tempo reale della carotide comune.

Il Capitolo 5 descrive un metodo, basato sul filtro sustain-attack, per la stima di orientamento e curvatura locali della carotide comune, in combinazione con un filtraggio direzionale delle pareti arteriose nell'immagine ad ultrasuoni per ottenere una migliore precisione nella stima del diametro esterno. II metodo è testato su 30 acquisizioni ultrasonografiche effettuate su 10 volontari, e rileva correttamente orientamento, curvatura e diametro per ogni soggetto considerato. La deviazione standard della posizione stimata delle pareti arteriose diminuisce significativamente quando il filtraggio direzionale viene applicato. Inoltre, l'algoritmo permette di correggere i valori di diametro in base all'orientamento locale dell'arteria.

Il Capitolo 6 presenta una metodologia automatica per la stima combinata delle forme d'onda temporali di diametro interno ed esterno della carotide comune, così come quelle relative all'IMT di entrambe le pareti arteriose visibili nello scan longitudinale. L'algoritmo, basato sul filtro sustain-attack e sul "baricentro anisotropo multiscala" intodotto nella presente tesi, cerca iterativamente la transizione lume-intima a partire dal centro del lume. Una misura automatica della qualità dell'angolo di inidenza tra il fascio ultrasonico e le pareti arteriose è codificata nei colori dei contorni sovrapposti all'immagine. La validazione è effettuata su 36 acquisizioni provenienti da 12 soggetti, così come su immagini sintetiche. I risultati indicano accuratezza e precisione elevate, con variazioni intraacquisizione in media inferiori a $50 \mu \mathrm{m}$ per diametro esterno ed IMT, ed inferiori a $100 \mu \mathrm{m}$ per il diametro interno. Un miglioramento della precisione è molto rilevante per un parametro come I'IMT, che presenta incrementi nell'ordine di $10 \mu \mathrm{m}$ all'anno. Il controllo dell'incidenza del fascio ultrasonico aumenta significativamente 
la ripetibilità delle stime di IMT, ed è stato sviluppato per perettere agli operatori di sistemi ad ultrasuoni di mantenere un piano di sezione corretto durante l'acquisizione. Ciò minimizza l'intererenza di fattori confondenti nell'interpretazione dei risultati delle stime ultrasonografiche non invasive.

Discussione e conclusioni della tesi sono contenute nel Capitolo 7. Si conclude constatando che gli algoritmi automatici introdotti riducono efficacemente la variabilità dei risultati, e permettono di neutralizzare fattori che tipicamente introducono rumore nell'imaging ultrasonografico, per esempio lo "speckle pattern" o le strategie di presentazione dell'immagine. Ciò può rivelarsi molto utile per facilitare il confronto di risultati provenienti da diversi sudi clinici, e può permettere che test su diverse popolazioni cliniche abbiano bisogno di un minor numero di soggetti analizzati per raggiungere rilevanza statistica. I metodi presentati in questa tesi permettono un'adeguata standardizzazione dei protocolli ultrasonografici per la stima delle proprietà morfologiche e dinamiche della carotide comune. L'automazione degli esami di routine sulla carotide comune, riguardante posizione, orientamento, delineazione delle pareti e controllo del piano di sezione, dà un feedback migliorato agli operatori, permettendo loro di concentrarsi appieno sull'appropriata acquisizione delle immagini ed in questo modo aumentando la robustezza delle procedure di stima ultrasonografica non invasiva di parametri cardiovascolari. 



\section{Thanks to...}

In these few lines of text I would like to acknowledge the persons thanks to whom the research I carried out has been finally shaped to form this doctoral dissertation.

First and foremost, I would like to express my most sincere gratitude to my supervisor Prof. Arnold Hoeks and co-supervisor Dr. Peter Brands for their constant and continuous support. Whenever I had a doubt or a question, they were always ready to listen to me and answer, I really appreciated that. Arnold, your insightful guidance and mentoring have been of immense value, and helped me to address the design of ultrasound processing methods from broader perspectives. Peter, thank you for your precious advices and suggestions regarding the development of the algorithms, for keeping me focused and on track day by day, and for the many fruitful discussions.

I would also like to thank Marcello Mazzarella and Joop Geijsen at Esaote Europe, and Cor van der Flier at Pie Medical Benelux, for being so supportive and kind, and for their great professionalism.

My gratitude goes to the members of the assessment committee for taking the time to read this manuscript and for their valuable comments and suggestions: Prof. Harry Struijker Boudier, Prof. Pierre Boutouyrie, Prof. Werner Mess, Prof. Robert Reneman, Prof. Patrick Segers. Thanks to Dr. Isabelle Ferreira, Dr. Raffaele Dellacà and Prof. Tammo Delhaas for accepting to be members of the "corona". I would also like to thank Prof. Reneman, Prof. Boutouyrie and Prof. Segers, as well as Prof. Frans van der Vosse, Dr. Marcel Rutten, Dr. Mariëlle Bosboom, Prof Luc Van Bortel, Dr. Anna Paini, Dr. Francesco Faita, Dr. Elisabetta Bianchini, Prof. Theo Arts, Dr. Peter Bovendeerd, Prof. Frits Prinzen, Dr. Nico Kuijpers, Prof. Marc van Zandvoort, Dr. Bart Beulen and Erwan Bozec for the numerous insightful discussions we had during the last four years.

Many thanks to my former supervisors at the TBM Lab of Milan Polytechnic University, Prof. Antonio Pedotti and Dr. Raffaele Dellacà, for their support during my master phase, and for guiding me towards the start of this PhD study in Maastricht. I also thank Dr. Andrea Aliverti and Dr. Christian Forlani for their advices.

I was very glad and honoured to be part of a team of "Marie Curie Fellows" on "Evaluation of Vascular Risk Predictors". Carole, Mihai and Nazia, you have been 
both dear friends and esteemed colleagues during this challenging period. Thank you for all the joyful moments we passed together, either discussing science or just "hanging out" with a drink. I am very happy to have met all of you.

A big "Grazie!" to my "Paranimfen", Dr. Koen Reesink and Dr. Steven de Jong, and to their girlfriends Danielle and Myrna. Koen, thanks for all the chats about biomedical engineering and music, there was always so much to say! And I really enjoyed the groovy jams we did on guitar and drums. Steven, it was a pleasure to share opinions on science and music fruition with you. Though our scientific areas are not likely to develop in future collaborations, it's nice to know that there is one thing we will always converge on: Porcupine Tree!

Thanks to my former colleagues at Esaote Europe for the enjoyable times they made me have inside and outside the company, as well for being so professional. Calin, Marco, Alex, David, Jean C., Tom, Danielle S., Carol J., Marina, Arie, Jo M., Perry, Meredith, Radou, Vincent, Bert P., Bert V., Natasha, Resie, Karin, Anouk, Jean V., Jo H., Cecile, Peter H., Jac, Peter K., Chris, Leon, Jean W., Max, Marc H., Gerry, Patrick V., Thijs, Dolf, Ralph, Arno, Rob C., Cindy, Ton and Anton. Thanks also to all the stagers I met in my former office: Maurice, Kevin, Sander, Werner, Maurice W., Alexandre, Stefan, Benjamin, Irene Z., Roel, Jeffrey. My gratitude also goes to Stevan Stuit and Maggie Kooman at Pie Medical Benelux.

Many thanks to all my former and current colleagues at Maastricht University, for being so friendly and encouraging: Evelien, Liselotte, Dennis, Jeroen, lulia, Patrick J., Lenneke, Wim, Anneke, Wilco, Joost, Irene, Jos, Ben, Ali, Kim, Mark, dank jullie wel!

I also want to thank the numerous friends with which I have been having lots of fun during these past four years: Sander B., Candida, Sander S., Svenja, Guillaume, Martin, Esther, Anca, Ousmane, Tizzo, Vale B., Pitto, Adry, Mike, Chiara, Becce, Simo, Elisa, Arx, Filippo, Lele, Prigio, Andrea, Cello, Pedro, Fabio, Nina, Gaia F., Zucca, Franzo, Vale S., Braghi, Lusi, Claudia, Daniele, Claudia N., Paolo N., Leonardo, Sara R., Pasquale, Emanuela Z., Alessandro G., Antonella, Gianluca, Giulia S., Mauro S., Antonia, Enrique, Irene A., Alberto B., Marta Z., Giovanni, Francesca P., Giorgio G., Joey, Kim V., Patrick K., Bart O., Melida, Betigou, Andreas, Tuuli, Joel, Laura, Anne B., Gijs, Daria, Marta W., Tim, Els, Robin, Ruben, Lisa, Franziska, Jana, Fenna, Iris, Sara M., Silvia, Luuk, Koos, Kookie, Sjoerd, Twanny. Vi ringrazio tutti.

Finally, love and gratitude to my sister Stefania, my mother Teresa, my father Guido, my aunts Patrizia, Giovanna, and Ines, and to the rest of my family, for being always close to me even from miles away. Siete voi ad avermi motivato e sostenuto in questi ultimi quattro anni. Grazie di cuore. 


\section{About the author}

Alessandro C. Rossi was born in Piacenza, Italy, on $9^{\text {th }}$ June 1981.

He obtained the bachelor degree in biomedical engineering from Milan Polytechnic University in 2003, with a thesis on non-invasive estimation of respiratory mechanics. In 2005 he graduated as a master of science in biomedical engineering from the same university with a master thesis on automatic segmentation of ultrasound images. From 2005 to 2009 he worked as a PhD candidate at Esaote Europe BV in Maastricht, The Netherlands, supported by a Marie Curie Early Stage Research Training Fellowship, and in cooperation with Maastricht University. His research focused on algorithms for non-invasive estimation of common carotid artery morphology and dynamics with ultrasound, and led to this PhD dissertation. In June 2009 he assumed the position of Post-Doc researcher at the Biomedical Engineering Department of Maastricht University. His current research involves the analysis of cardiac structure-function relation based on magnetic resonance imaging.

Alessandro has a strong passion for both listening and performing music, in many different as well as antithetical forms. 


\section{Publications}

\section{Scientific Journals}

Rossi AC, Brands PJ, Hoeks APG. Automatic recognition of the common carotid artery in longitudinal ultrasound B-mode scans. Medical Image Analysis 2008; 12: 653-665.

Rossi AC, Brands PJ, Hoeks APG. Nonlinear processing in B-mode ultrasound affects carotid diameter assessment. Ultrasound in Medicine and Biology 2009; 35: 736-747.

Rossi AC, Brands PJ, Hoeks APG. Automatic localization of intimal and adventitial layers with non-invasive ultrasound: a novel algorithm providing scan quality control. 2009. Submitted for publication.

\section{Conference Proceedings}

Rossi AC, Brands PJ, Hoeks APG. Fully automatic assessment of carotid artery curvature and diameter with non-invasive ultrasound. IFMBE Conference Proceedings. Antwerp, Belgium, November 23-27, 2008; 22: 588-591.

Zaccari I, Rossi AC, Bosboom EMH, Brands PJ. Pressure waveform estimation in the common carotid artery - Different methods in comparison. IFMBE Conference Proceedings. Antwerp, Belgium, November 23-27, 2008; 22: 2536-2539.

\section{Abstracts}

Rossi AC, Brands PJ, Hoeks APG. A new automatic artery lumen identification algorithm for carotid ultrasound images. Marie Curie MC2 Conference, Manchester, United Kingdom, April 10-12, 2006.

Rossi AC, Brands PJ, Hoeks APG. A novel automatic lumen identification algorithm for ultrasound B-mode scans of the carotid artery. IEEE International Ultrasonic Symposium, Vancouver, Canada, October 3-6, 2006.

Zaccari I, Rossi AC, Bosboom EMH, Brands PJ. Pressure waveform estimation in the common carotid artery - Different methods in comparison. Artery 7 Conference, Prague, Czech Republic, September 14-15, 2007. 


\section{Color figures}
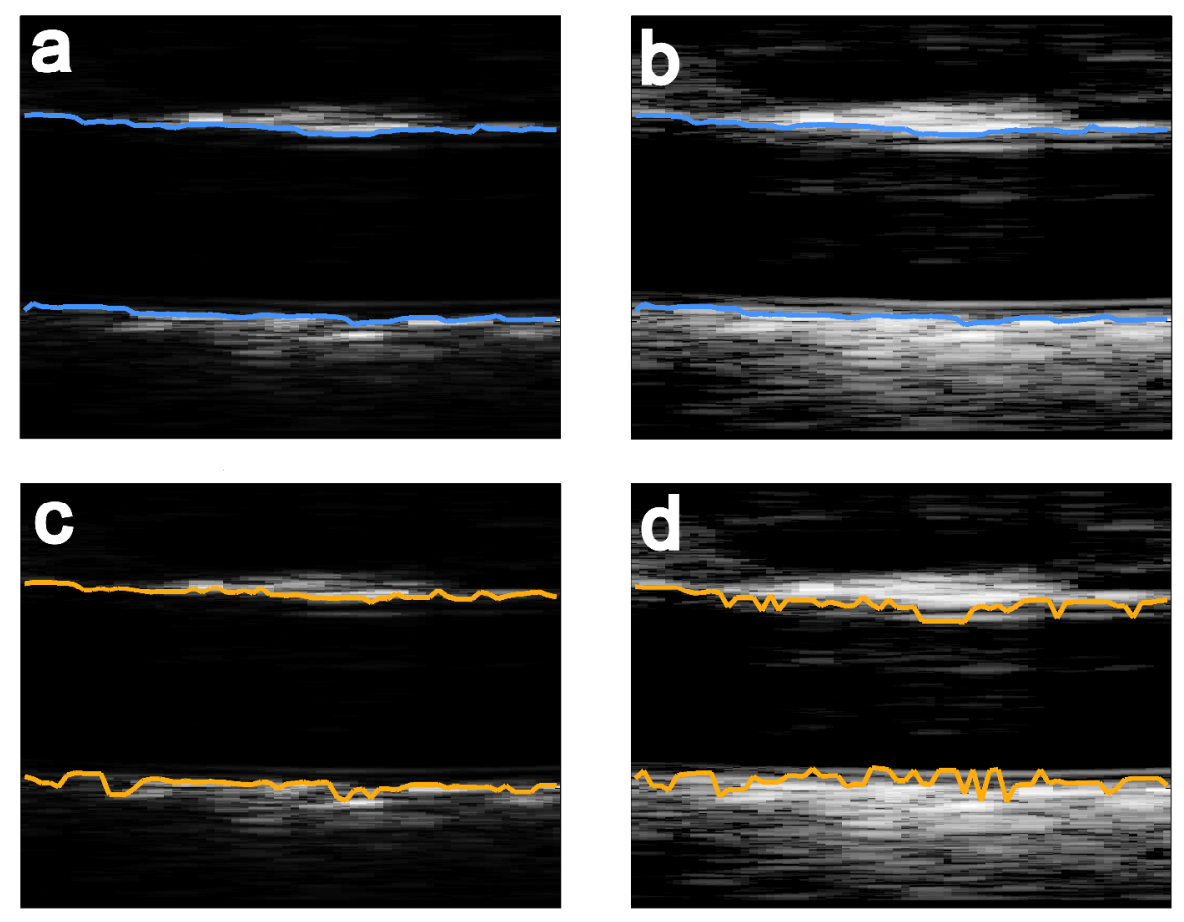

Fig. 3.5 (Chapter 3, page 49) - a) and b) Sustain-attack filter (blue lines) applied to a linear and a log-compressed image, respectively. c) and d) Derivative approach (orange lines) applied to a linear and a log-compressed image, respectively. All images are unsaturated, and refer to a $\mathrm{ROI}$ consisting of 64 lines of sight, centered on the carotid lumen and covering a depth of 16 $\mathrm{mm}$. No post-processing was performed on the results, in order to properly assess the effect of different pre-processing conditions on the outcome of the two basic edge detectors. 

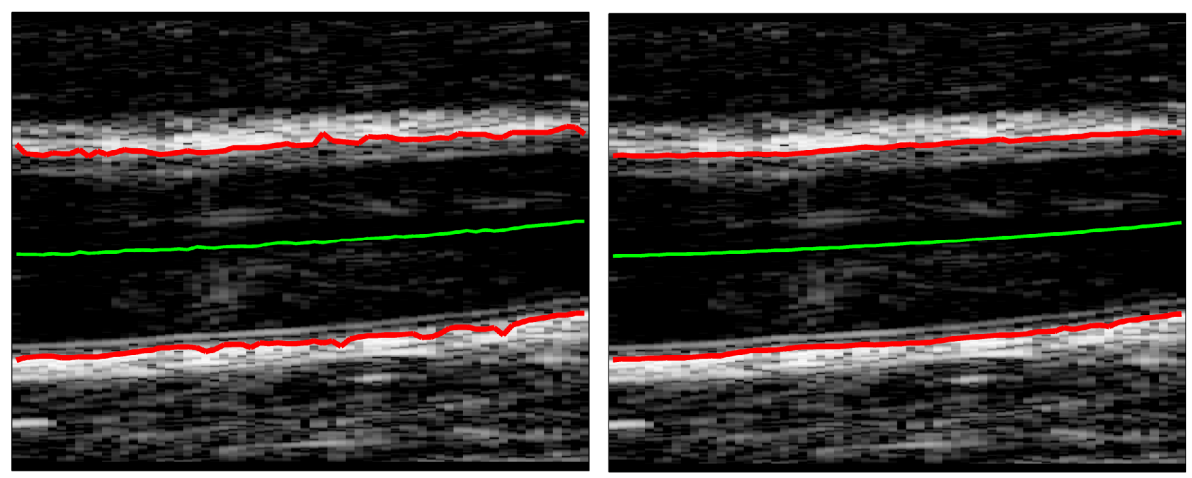

Fig. 5.5 (Chapter 5, page 92) - Left: no directional smoothing; right: results obtained with directional smoothing, remapped on the original ROI. The red lines are obtained with the sustain-attack filter. The processing occurs on linear envelopes, but here images are shown with logarithmic compression for visual convenience. 


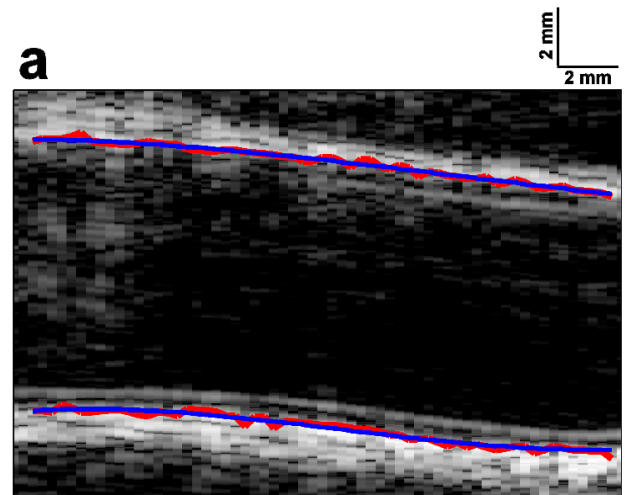

b

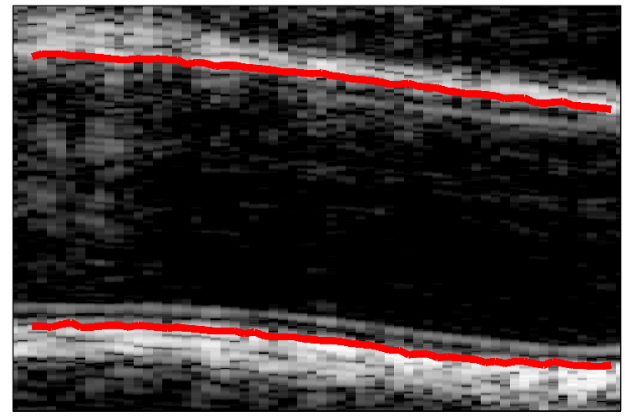

C

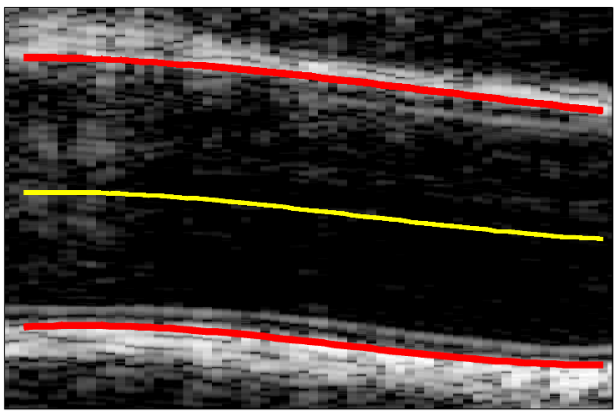

Fig. 6.2 (Chapter 6, page 100) - Delineation of adventitial contours and centerline. a) The outcome of the sustain-attack filter (red lines) is fitted with a third-order polynomial (blue lines); b) outlier rejection is performed; c) an additional third-order fit is performed (red lines) in order to smoothly follow the arterial curvature; the centerline (yellow line) follows from the anterior and posterior wall positions. 


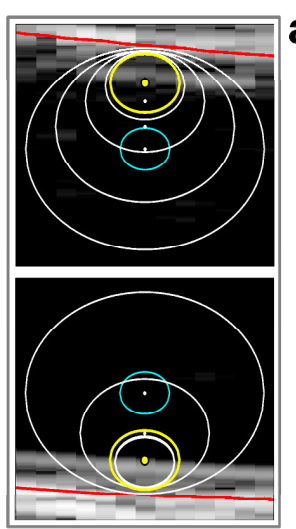

d

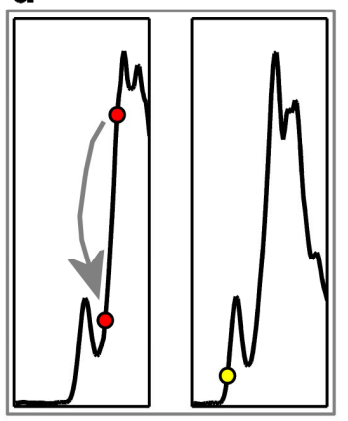

b

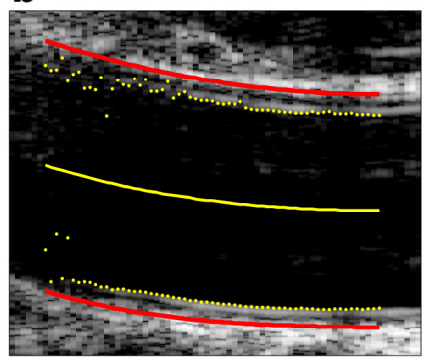

C

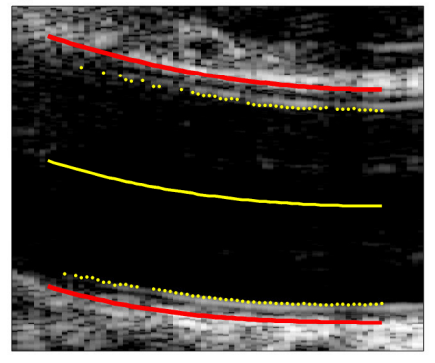

e

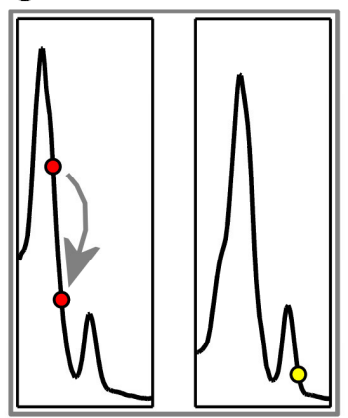

f

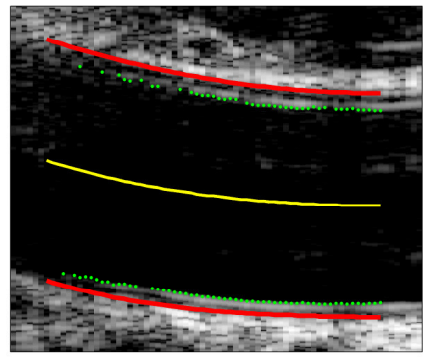

Fig. 6.3 (Chapter 6, page 103) - a) MAB method acting across one vertical line; top panel: near wall; bottom panel: far wall; the initial seed points are placed at $2 \mathrm{~mm}$ from the CCA centreline. The evaluation domain (white circles) changes size at each iteration according to the previously detected adventitial position. The smoothing domain (blue circles, here shown only at the first iteration for visual convenience) extends $716 \mu \mathrm{m}$ in depth and $945 \mu \mathrm{m}$ in width. The yellow circles/points refer to the final iteration. b) The algorithm acts on the 61 centremost signals in the ROI. c) Intimal contour outliers are rejected. In this example the IMT unbiasing procedure involves equalization, subsequent to re-alignment and lateral averaging, for far wall adventitia ( $d$, left panel) with respect to far wall intima (d, right panel), and for near wall adventitia (e, left panel) with respect to near wall intima (e, right panel). f) Final result of the automatic CCA segmentation. 


\section{a}

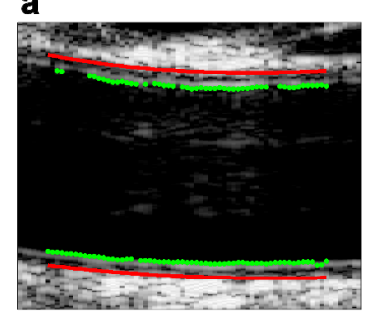

\section{d}

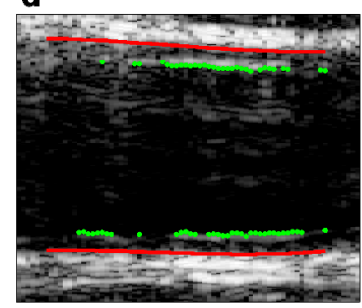

\section{g}

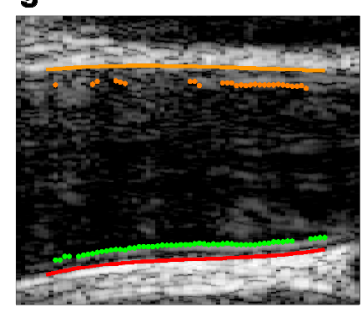

b

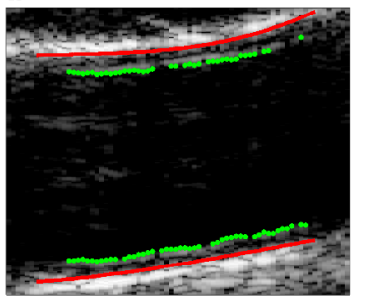

e

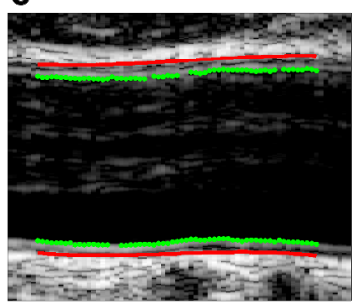

h

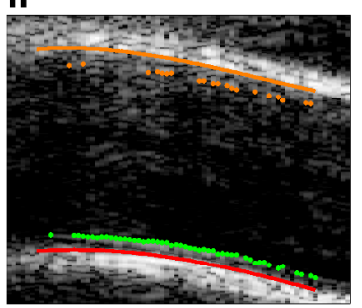

c

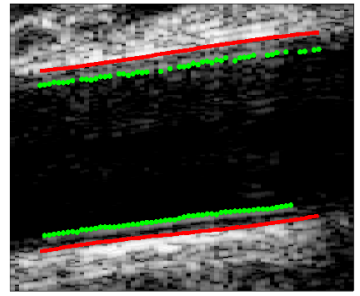

f
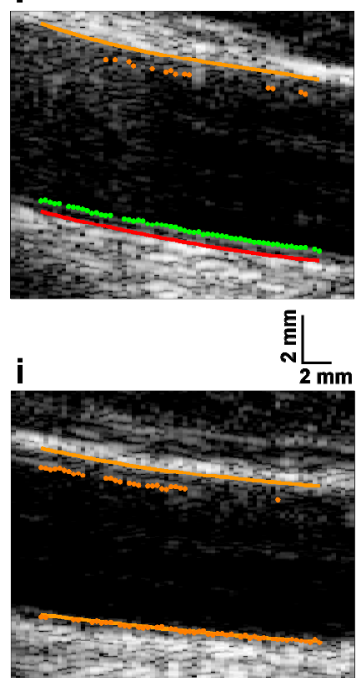

Fig. 6.4 (Chapter 6, page 105) - Examples of automatic CCA segmentation. An "in-plane" condition is symbolized by a green intimal contour and a red adventitial contour. When the automatic scan plane control yields an "out plane" result, the contours are displayed in orange. 
